\title{
Evolutionary game theory: Temporal and spatial effects beyond replicator dynamics
}

\author{
Carlos P. Roca ${ }^{\text {a,* }}$, José A. Cuesta ${ }^{\text {a }}$, Angel Sánchez ${ }^{\text {a,b,c }}$ \\ ${ }^{a}$ Grupo Interdisciplinar de Sistemas Complejos (GISC), Departamento de Matemáticas, Universidad Carlos III de Madrid, \\ Avenida de la Universidad 30, 28911 Leganés, Madrid, Spain \\ b Instituto de Biocomputación y Física de Sistemas Complejos (BIFI), Universidad de Zaragoza, Corona de Aragón 42, 50009 Zaragoza, Spain \\ ${ }^{\mathrm{c}}$ Instituto de Ciencias Matemáticas CSIC-UAM-UC3M-UCM, 28006 Madrid, Spain
}

\begin{abstract}
Evolutionary game dynamics is one of the most fruitful frameworks for studying evolution in different disciplines, from Biology to Economics. Within this context, the approach of choice for many researchers is the so-called replicator equation, that describes mathematically the idea that those individuals performing better have more offspring and thus their frequency in the population grows. While very many interesting results have been obtained with this equation in the three decades elapsed since it was first proposed, it is important to realize the limits of its applicability. One particularly relevant issue in this respect is that of non-meanfield effects, that may arise from temporal fluctuations or from spatial correlations, both neglected in the replicator equation. This review discusses these temporal and spatial effects focusing on the non-trivial modifications they induce when compared to the outcome of replicator dynamics. Alongside this question, the hypothesis of linearity and its relation to the choice of the rule for strategy update is also analyzed. The discussion is presented in terms of the emergence of cooperation, as one of the current key problems in Biology and in other disciplines.
\end{abstract}

PACS: 02.50.Le; 05.40.-a; 87.23.Ge; 87.23.Kg; 89.65.-s; 89.75.Fb

MSC: 91A22; 91A43; 92D15

Keywords: Evolutionary games; Replicator dynamics; Mean-field; Fluctuations; Spatial structure; Network reciprocity; Emergence of cooperation

\section{Contents}

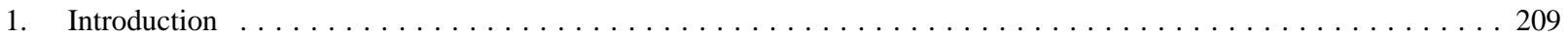

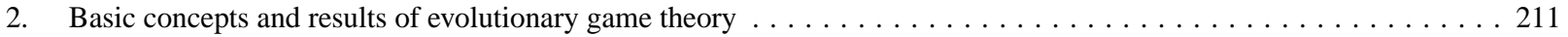

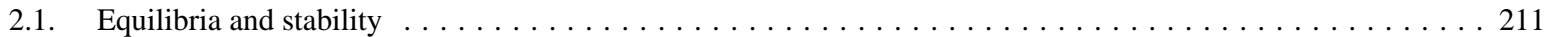

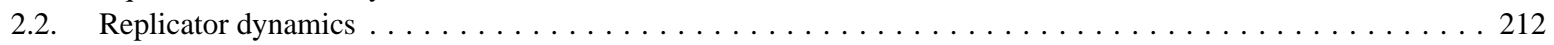

\footnotetext{
* Corresponding author.

E-mail addresses: cproca@math.uc3m.es (C.P. Roca), cuesta@math.uc3m.es (J.A. Cuesta), anxo@math.uc3m.es (A. Sánchez).
} 


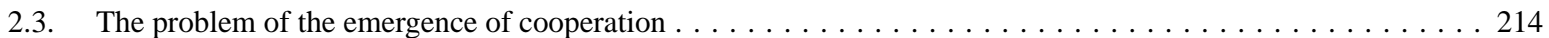

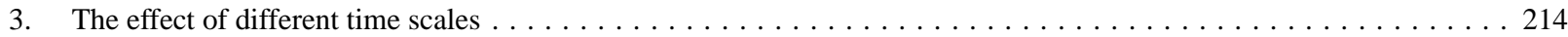

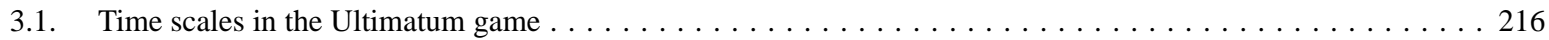

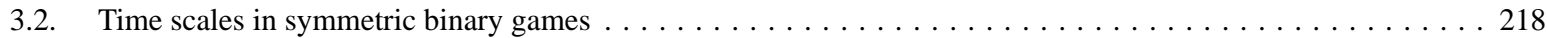

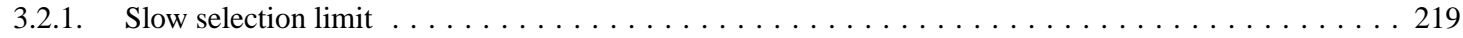



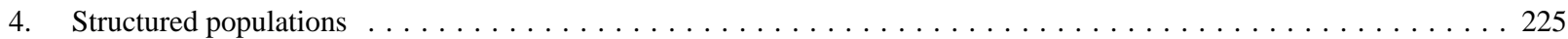

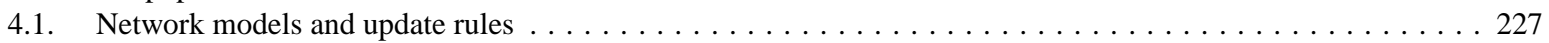

4.2. Spatial structure and homogeneous networks $\ldots \ldots \ldots \ldots \ldots \ldots$

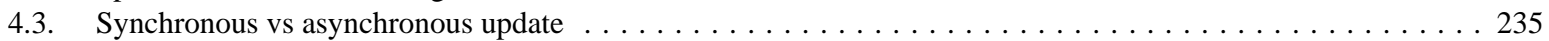

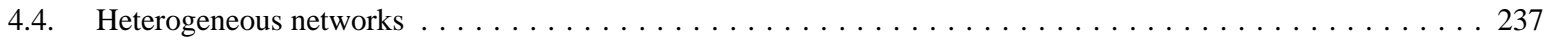

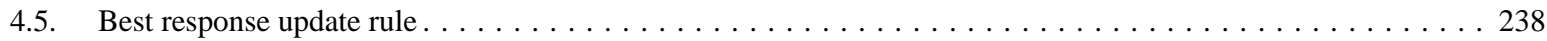

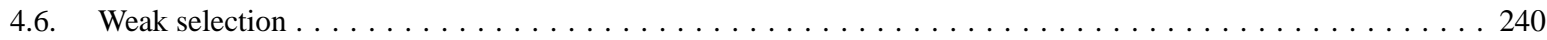

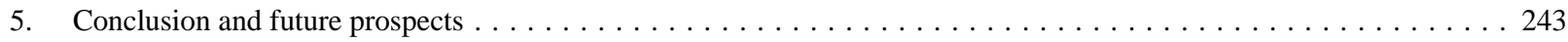

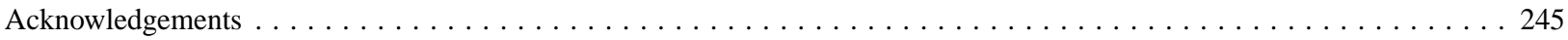

Appendix A. Characterization of birth-death processes $\ldots \ldots \ldots \ldots \ldots \ldots \ldots$

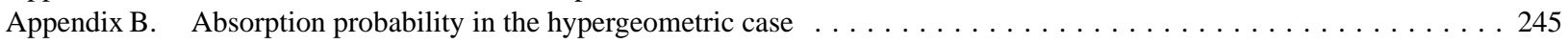

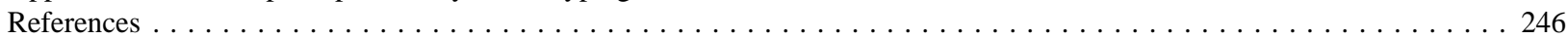

\section{Introduction}

The importance of evolution can hardly be overstated, in so far as it permeates all sciences. Indeed, in the 150 years that have passed since the publication of On the Origin of the Species [1], the original idea of Darwin that evolution takes place through descent with modification acted upon by natural selection has become a key concept in many sciences. Thus, nowadays one can speak of course of evolutionary biology, but there are also evolutionary disciplines in economics, psychology, linguistics, or computer science, to name a few.

Darwin's theory of evolution was based on the idea of natural selection. Natural selection is the process through which favorable heritable traits become more common in successive generations of a population of reproducing organisms, displacing unfavorable traits in the struggle for resources. In order to cast this process in a mathematically precise form, J.B.S. Haldane and Sewall Wright introduced, in the so-called modern evolutionary synthesis of the 1920 's, the concept of fitness. They applied theoretical population ideas to the description of evolution and, in that context, they defined fitness as the expected number of offspring of an individual that reach adulthood. In this way they were able to come up with a well-defined measure of the adaptation of individuals and species to their environment.

The simplest mathematical theory of evolution one can think of arises when one assumes that the fitness of a species does not depend on the distribution of frequencies of the different species in the population, i.e., it only depends on factors that are intrinsic to the species under consideration or on environmental influences. Sewall Wright formalized this idea in terms of fitness landscapes ca. 1930, and in that context R. Fisher proved his celebrated theorem, that states that the mean fitness of a population is a non-decreasing function of time, which increases proportionally to variability. Since then, a lot of work has been done on this kind of models; we refer the reader to [2-5] for reviews.

The approach in terms of fitness landscapes is, however, too simple and, in general, it is clear that the fitness of a species will depend on the composition of the population and will therefore change accordingly as the population evolves. If one wants to describe evolution at this level, the tool of reference is evolutionary game theory. Brought into biology by Maynard Smith [6] as an "exaptation"1 of the game theory developed originally for economics [8], it has since become a unifying framework for other disciplines, such as sociology or anthropology [9]. The key feature of this mathematical apparatus is that it allows to deal with evolution on a frequency-dependent fitness landscape or, in other words, with strategic interactions between entities, these being individuals, groups, species, etc. Evolutionary game theory is thus the generic approach to evolutionary dynamics [10] and contains as a special case constant, or fitness landscape, selection.

1 Borrowing the term introduced by Gould and Vrba in evolutionary theory, see [7]. 
In its thirty year history, a great deal of research in evolutionary game theory has focused on the properties and applications of the replicator equation [11]. The replicator equation was introduced in 1978 by Taylor and Jonker [12] and describes the evolution of the frequencies of population types taking into account their mutual influence on their fitness. This important property allows the replicator equation to capture the essence of selection and, among other key results, it provides a connection between the biological concept of evolutionarily stable strategies [6] with the economical concept of Nash equilibrium [13].

As we will see below, the replicator equation is derived in a specific framework that involves a number of assumptions, beginning with that of an infinite, well-mixed population with no mutations. By well-mixed population it is understood that every individual either interacts with every other one or at least has the same probability to interact with any other individual in the population. This hypothesis implies that any individual effectively interacts with a player which uses the average strategy within the population (an approach that has been traditionally used in physics under the name of mean-field approximation). Deviations from the well-mixed population scenario affect strongly and non-trivially the outcome of the evolution, in a way which is difficult to apprehend in principle. Such deviations can arise when one considers, for instance, finite size populations, alternative learning/reproduction dynamics, or some kind of structure (spatial or temporal) in the interactions between individuals.

In this review we will focus on this last point, and discuss the consequences of relaxing the hypothesis that every player interacts or can interact with every other one. We will address both spatial and temporal limitations in this paper, and refer the reader to Refs. [10,11] for discussions of other perturbations. For the sake of definiteness, we will consider those effects, that go beyond replicator dynamics, in the specific context of the emergence of cooperation, a problem of paramount importance with implications at all levels, from molecular biology to societies and ecosystems [14]; many other applications of evolutionary dynamics have also been proposed but it would be too lengthy to discuss all of them here (the interested reader should see, e.g., [10]). Cooperation, understood as a fitness-decreasing behavior that increases others' fitness, is an evolutionary puzzle, and many researchers have considered alternative approaches to the replicator equation as possible explanations of its ubiquity in human (and many animal) societies. As it turns out, human behavior is unique in nature. Indeed, altruism or cooperative behavior exists in other species, but it can be understood in terms of genetic relatedness (kin selection, introduced by Hamilton $[15,16]$ ) or of repeated interactions (as proposed by Trivers [17]). Nevertheless, human cooperation extends to genetically unrelated individuals and to large groups, characteristics that cannot be understood within those schemes. Subsequently, a number of theories based on group and/or cultural evolution have been put forward in order to explain altruism (see [18] for a review). Evolutionary game theory is also being intensively used for this research, its main virtue being that it allows to pose the dilemmas involved in cooperation in a simple, mathematically tractable manner. To date, however, there is not a generally accepted solution to this puzzle [19].

Considering temporal and spatial effects means, in the language of physics, going beyond mean-field to include fluctuations and correlations. Therefore, a first step is to understand what are the basic mean field results. To this end, in Section 2 we briefly summarize the main features of replicator equations and introduce the concepts we will refer to afterwards. Subsequently, Section 3 discusses how fluctuations can be taken into account in evolutionary game theory, and specifically we will consider that, generically, interactions and dynamics (evolution) need not occur at the same pace. We will show that the existence of different time scales leads to quite unexpected results, such as the survival and predominance of individuals that would be the less fit in the replicator description. For games in finite populations with two types of individuals or strategies, the problem can be understood in terms of Markov processes and the games can be classified according to the influence of the time scales on their equilibrium structure. Other situations can be treated by means of numerical simulations with similarly non-trivial results.

Section 4 deals with spatial effects. The inclusion of population structure in evolutionary game theory has been the subject of intense research in the last 15 years, and a complete review would be beyond our purpose (see e.g. [20]). The existence of a network describing the possible interactions in the population has been identified as one of the factors that may promote cooperation among selfish individuals [19]. We will discuss the results available to date and show how they can be reconciled by realizing the role played by different networks, different update rules for the evolution of strategies and the equilibrium structure of the games. As a result, we will be able to provide a clear-cut picture of the extent as to which population structure promote cooperation in two strategy games.

Finally, in Section 5 we discuss the implications of the reviewed results on a more general context. Our major conclusion will be the lack of generality of models in evolutionary game theory, where details of the dynamics and the interaction modify qualitatively the results. A behavior like that is not intuitive to physicists, used to disregard 
those details as unimportant. Therefore, until we are able to discern what is and what is not relevant, when dealing with problems in other sciences, modeling properly and accurately specific problems is of utmost importance. We will also indicate a few directions of research that arise from the presently available knowledge and that we believe will be most appealing in the near future.

\section{Basic concepts and results of evolutionary game theory}

In this section, we summarize the main facts about evolutionary game theory that we are going to need in the remainder of the paper. The focus is on the stability of strategies and on the replicator equation, as an equivalent to the dynamical description of a mean field approach which we will be comparing with. This summary is by no means intended to be comprehensive and we encourage the reader to consult the review [21] or, for full details, the books [6, $9,11]$.

\subsection{Equilibria and stability}

The simplest type of game has only two players and, as this will be the one we will be dealing with, we will not dwell into further complications. Player $i$ is endowed with a finite number $n_{i}$ of strategies. A game is defined by listing the strategies available to the players and the payoffs they yield: When a player, using strategy $s_{i}$, meets another, who in turn uses strategy $s_{j}$, the former receives a payoff $W_{i j}$ whereas the latter receives a payoff $Z_{i j}$. We will restrict ourselves to symmetric games, in which the roles of both players are exchangeable (except in the example considered in Section 3.1); mathematically, this means that the set of strategies are the same for both players and that $W=Z^{T}$. Matrix $W$ is then called the payoff matrix of the normal form of the game. In the original economic formulation [8] payoffs were understood as utilities, but Maynard Smith [6] reinterpreted them in terms of fitness, i.e. in terms of reproductive success of the involved individuals.

The fundamental step to "solving" the game or, in other words, to find what strategies will be played, was put forward by John Nash [13] by introducing the concept of equilibrium. In $2 \times 2$ games, a pair of strategies $\left(s_{i}, s_{j}\right)$ is a Nash equilibrium if no unilateral change of strategy allows any player to improve her payoff. When we restrict ourselves to symmetric games, one can say simply, by an abuse of language [21], that a strategy $s_{i}$ is a Nash equilibrium if it is a best reply to itself: $W_{i i} \geqslant W_{i j}, \forall s_{j}$ (a strict Nash equilibrium if the inequality is strict). This in turn implies that if both players are playing strategy $s_{i}$, none of them has any incentive to deviate unilaterally by choosing other strategy. As an example, let us consider the famous Prisoner's Dilemma game, which we will be discussing throughout the review. Prisoner's Dilemma was introduced by Rapoport and Chammah [22] as a model of the implications of nuclear deterrence during the Cold War, and is given by the following payoff matrix (we use the traditional convention that the matrix indicates payoffs to the row player)

$$
\begin{gathered}
\text { C } \\
\text { C } \\
\text { D }
\end{gathered}\left(\begin{array}{ll}
3 & 0 \\
5 & 1
\end{array}\right) .
$$

The strategies are named C and D for cooperating and defecting, respectively. This game is referred to as Prisoner's Dilemma because it is usually posed in terms of two persons that are arrested accused of a crime. The police separates them and makes the following offer to them: If one confesses and incriminates the other, she will receive a large reduction in the sentence, but if both confess they will only get a minor reduction; and if nobody confesses then the police is left only with circumstancial evidence, enough to imprison them for a short period. The amounts of the sentence reductions are given by the payoffs in (1). It is clear from it that $\mathrm{D}$ is a strict Nash equilibrium: To begin with, it is a dominant strategy, because no matter what the column player chooses to do, the row player is always better off by defecting; and when both players defect, none will improve her situation by cooperating. In terms of the prisoners, this translates into the fact that both will confess if they behave rationally. The dilemma arises when one realizes that both players would be better off cooperating, i.e. not confessing, but rationality leads them unavoidable to confess.

The above discussion concerns Nash equilibria in pure strategies. However, players can also use the so-called mixed strategies, defined by a vector with as many entries as available strategies, every entry indicating the probability of using that strategy. The notation changes then accordingly: We use vectors $\mathbf{x}=\left(x_{1} x_{2} \ldots x_{n}\right)^{T}$, which are elements 
of the simplex $S_{n}$ spanned by the vectors $\mathbf{e}_{i}$ of the standard unit base (vectors $\mathbf{e}_{i}$ are then identified with the $n$ pure strategies). The definition of a Nash equilibrium in mixed strategies is identical to the previous one: The strategy profile $\mathbf{x}$ is a Nash equilibrium if it is a best reply to itself in terms of the expected payoffs, i.e., if $\mathbf{x}^{T} W \mathbf{x} \geqslant \mathbf{x}^{T} W \mathbf{y}, \forall \mathbf{y} \in S_{n}$. Once mixed strategies have been introduced, one can prove, following Nash [13] that every normal form game has at least one Nash equilibrium, albeit it need not necessarily be a Nash equilibrium in pure strategies. An example we will also be discussing below is given by the Hawk-Dove game (also called Snowdrift or Chicken in the literature [23]), introduced by Maynard Smith and Price to describe animal conflicts [24], whose payoff matrix is e.g. (strategies are labeled $\mathrm{H}$ and $\mathrm{D}$ for Hawk and Dove, respectively)

$$
\begin{gathered}
\text { D } \\
\text { D } \\
\text { H } \\
\left.\begin{array}{cc}
3 & 1 \\
5 & 0
\end{array}\right) .
\end{gathered}
$$

In this case, neither H nor D are Nash equilibria, but there is indeed one Nash equilibrium in mixed strategies, that can be shown [6] to be given by playing $\mathrm{D}$ with probability $1 / 3$. This makes sense in terms of the meaning of the game, which is an anti-coordination game, i.e. the best thing to do is the opposite of the other player. Indeed, in the Snowdrift interpretation, two people are trapped by a snowdrift at the two ends of a road. For every one of them, the best option is not to shovel snow off to free the road and let the other person do it; however, if the other person does not shovel, then the best option is to shovel oneself. There is, hence, a temptation to defect that creates a dilemmatic situation (in which mutual defection leads to the worst possible outcome).

In the same way as he reinterpreted monetary payoffs in terms of reproductive success, Maynard Smith reinterpreted mixed strategies as population frequencies. This allowed to leave behind the economic concept of rational individual and move forward to biological applications (as well as in other fields). As a consequence, the economic evolutionary idea in terms of learning new strategies gives way to a genetic transmission of behavioral strategies to offspring. Therefore, Maynard Smith's interpretation of the above result is that a population consisting of one third of individuals that always use the D strategy and two thirds of H-strategists is a stable genetic polymorphism. At the core of this concept is his notion of evolutionarily stable strategy. Maynard Smith defined a strategy as evolutionarily stable if the following two conditions are satisfied

$$
\begin{aligned}
& \mathbf{x}^{T} W \mathbf{x} \geqslant \mathbf{x}^{T} W \mathbf{y}, \quad \forall \mathbf{y} \in S_{n}, \\
& \text { if } \mathbf{x} \neq \mathbf{y} \text { and } \mathbf{x}^{T} W \mathbf{y}=\mathbf{x}^{T} W \mathbf{x}, \quad \text { then } \mathbf{x}^{T} W \mathbf{y}>\mathbf{y}^{T} W \mathbf{y} .
\end{aligned}
$$

The rationale behind this definition is again of a population theoretical type: These are the conditions that must be fulfilled for a population of $\mathbf{x}$-strategists to be non-invadable by any $\mathbf{y}$-mutant. Indeed, either $\mathbf{x}$ performs better against itself than $\mathbf{y}$ or, if they perform equally, $\mathbf{x}$ performs better against $\mathbf{y}$ than $\mathbf{y}$ itself. These two conditions guarantee non-invasibility of the population. On the other hand, comparing the definitions of evolutionarily stable strategy and Nash equilibrium one can immediately see that a strict Nash equilibrium is an evolutionarily stable strategy and that an evolutionarily stable strategy is a Nash equilibrium.

\subsection{Replicator dynamics}

After Nash proposed his definition of equilibrium, the main criticism that the concept has received relates to how equilibria are reached. In other words, Nash provided a rule to decide which are the strategies that rational players should play in a game, but how do people involved in actual game-theoretical settings but without knowledge of game theory find the Nash equilibrium? Furthermore, in case there is more than one Nash equilibrium, which one should be played, i.e., which one is the true "solution" of the game? These questions started out a great number of works dealing with learning and with refinements of the concept that allowed to distinguish among equilibria, particularly within the field of economics. This literature is out of the scope of the present review and the reader is referred to [25] for an in-depth discussion.

One of the answers to the above criticism arises as a bonus from the ideas of Maynard Smith. The notion of evolutionarily stable strategy has implicit some kind of dynamics when we speak of invasibility by mutants; a population is stable if when a small proportion of it mutates it eventually evolves back to the original state. One could therefore expect that, starting from some random initial condition, populations would evolve to an evolutionarily stable strategy, 
which, as already stated, is nothing but a Nash equilibrium. Thus, we would have solved the question as to how the population "learns" to play the Nash equilibrium and perhaps the problem of selecting among different Nash equilibria. However, so far we have only spoken of an abstract dynamics; nothing is specified as to what is the evolution of the population or the strategies that it contains.

The replicator equation, due to Taylor and Jonker [12], was the first and most successful proposal of an evolutionary game dynamics. Within the population dynamics framework, the state of the population, i.e., the distribution of strategy frequencies, is given by $\mathbf{x}$ as above. A first key point is that we assume that the $x_{i}$ are differentiable functions of time $t$ : This requires in turn assuming that the population is infinitely large (or that $x_{i}$ are expected values for an ensemble of populations). Within this hypothesis, we can now postulate a law of motion for $\mathbf{x}(t)$. Assuming further that individuals meet randomly, engaging in a game with payoff matrix $W$, then $(W \mathbf{x})_{i}$ is the expected payoff for an individual using strategy $s_{i}$, and $\mathbf{x}^{T} W \mathbf{x}$ is the average payoff in the population state $\mathbf{x}$. If we, consistently with our interpretation of payoff as fitness, postulate that the per capita rate of growth of the subpopulation using strategy $s_{i}$ is proportional to its payoff, we arrive at the replicator equation (the name was first proposed in [26])

$$
\dot{x}_{i}=x_{i}\left[(W \mathbf{x})_{i}-\mathbf{x}^{T} W \mathbf{x}\right],
$$

where the term $\mathbf{x}^{T} W \mathbf{x}$ arises to ensure the constraint $\sum_{i} x_{i}=1\left(\dot{x}_{i}\right.$ denotes the time derivative of $\left.x_{i}\right)$. This equation translates into mathematical terms the elementary principle of natural selection: Strategies, or individuals using a given strategy, that reproduce more efficiently spread, displacing those with smaller fitness. Note also that states with $x_{i}=1, x_{j}=0, \forall j \neq i$ are solutions of Eq. (5) and, in fact, they are absorbing states, playing a relevant role in the dynamics of the system in the absence of mutation.

Once an equation has been proposed, one can resort to the tools of dynamical systems theory to derive its most important consequences. In this regard, it is interesting to note that the replicator equation can be transformed by an appropriate change of variable in a system of Lotka-Volterra type [11]. For our present purposes, we will focus only on the relation of the replicator dynamics with the two equilibrium concepts discussed in the preceding subsection. The rest points of the replicator equation are those frequency distributions $\mathbf{x}$ that make the rhs of Eq. (5) vanish, i.e. those that verify either $x_{i}=0$ or $(W \mathbf{x})_{i}=\mathbf{x}^{T} W \mathbf{x}, \forall i=1, \ldots, n$. The solutions of this system of equations are all the mixed strategy Nash equilibria of the game [9]. Furthermore, it is not difficult to show (see e.g. [11]) that strict Nash equilibria are asymptotically stable, and that stable rest points are Nash equilibria. We thus see that the replicator equation provides us with an evolutionary mechanism through which the players, or the population, can arrive at a Nash equilibrium or, equivalently, to an evolutionarily stable strategy. The different basins of attraction of the different equilibria further explain which of them is selected in case there are more than one.

For our present purposes, it is important to stress the hypothesis involved (explicitly or implicitly) in the derivation of the replicator equation:

1. The population is infinitely large.

2. Individuals meet randomly or play against every other one, such that the payoff of strategy $s_{i}$ is proportional to the payoff averaged over the current population state $\mathbf{x}$.

3. There are no mutations, i.e. strategies increase or decrease in frequency only due to reproduction.

4. The variation of the population is linear in the payoff difference.

Assumptions 1 and 2 are, as we stated above, crucial to derive the replicator equation in order to replace the fitness of a given strategy by its mean value when the population is described in terms of frequencies. Of course, finite populations deviate from the values of frequencies corresponding to infinite ones. In a series of recent works, Traulsen and co-workers have considered this problem [27-29]. They have identified different microscopic stochastic processes that lead to the standard or the adjusted replicator dynamics, showing that differences on the individual level can lead to qualitatively different dynamics in asymmetric conflicts and, depending on the population size, can even invert the direction of the evolutionary process. Their analytical framework, which they have extended to include an arbitrary number of strategies, provides good approximations to simulation results for very small sizes. For a recent review of these and related issues, see [30]. On the other hand, there has also been some work showing that evolutionarily stable strategies in infinite populations may lose their stable character when the population is small (a result not totally unrelated to those we will discuss in Section 3). For examples of this in the context of Hawk-Dove games, see [31,32]. 
Assumption 3 does not pose any severe problem. In fact, mutations (or migrations among physically separated groups, whose mathematical description is equivalent) can be included, yielding the so-called replicator-mutator equation [33]. This is in turn equivalent to the Price equation [34], in which a term involving the covariance of fitness and strategies appears explicitly. Mutations have been also included in the framework of finite size populations [29] mentioned above. We refer the reader to Refs. [33,35] for further analysis of this issue.

Assumption 4 is actually the core of the definition of replicator dynamics. In Section 4 below we will come back to this point, when we discuss the relation of replicator dynamics to the rules used for the update of strategies in agentbased models. Work beyond the hypothesis of linearity can proceed otherwise in different directions, by considering generalized replicator equations of the form

$$
\dot{x}_{i}=x_{i}\left[W_{i}(\mathbf{x})-\mathbf{x}^{T} W(\mathbf{x})\right] .
$$

The precise choice for the functions $W_{i}(\mathbf{x})$ depends of course on the particular situation one is trying to model. A number of the results on replicator equation carry on for several such choices. This topic is well summarized in [21] and the interested reader can proceed from there to the relevant references.

Assumption 2 is the one to which this review is devoted to and, once again, there are very many different possibilities in which it may not hold. We will discuss in depth below the case in which the time scale of selection is faster than that of interaction, leading to the impossibility that a given player can interact with all others. Interactions may be also physically limited, either for geographical reasons (individuals interact only with those in their surroundings), for social reasons (individuals interact only with those with whom they are acquainted) or otherwise. As in previous cases, these variations prevents one from using the expected value of fitness of a strategy in the population as a good approximation for its growth rate. We will see the consequences this has in the following sections.

\subsection{The problem of the emergence of cooperation}

One of the most important problems to which evolutionary game theory is being applied is the understanding of the emergence of cooperation in human (albeit non-exclusively) societies [14]. As we stated in the Introduction, this is an evolutionary puzzle that can be accurately expressed within the formalism of game theory. One of the games that has been most often used in connection with this problem is the Prisoner's Dilemma introduced above, Eq. (1). As we have seen, rational players should unavoidably defect and never cooperate, thus leading to a very bad outcome for both players. On the other hand, it is evident that if both players had cooperated they would have been much better off. This is a prototypical example of a social dilemma [36] which is, in fact, (partially) solved in societies. Indeed, the very existence of human society, with its highly specialized labor division, is a proof that cooperation is possible.

In more biological terms, the question can be phrased using again the concept of fitness. Why should an individual help other achieve more fitness, implying more reproductive success and a chance that the helper is eventually displaced? It is important to realize that such a cooperative effort is at the roots of very many biological phenomena, from mutualism to the appearance of multicellular organisms [37].

When one considers this problem in the framework of replicator equation, the conclusion is immediate and disappointing: Cooperation is simply not possible. As defection is the only Nash equilibrium of Prisoner's Dilemma, for any initial condition with a positive fraction of defectors, replicator dynamics will inexorably take the population to a final state in which they all are defectors. Therefore, one needs to understand how the replicator equation framework can be supplemented or superseded for evolutionary game theory to become closer to what is observed in the real world (note that there is no hope for classical game theory in this respect as it is based in the perfect rationality of the players). Relaxing the above discussed assumptions leads, in some cases, to possible solutions to this puzzle, and our aim here is to summarize and review what has been done along these lines with Assumption 2.

\section{The effect of different time scales}

Evolution is generally supposed to occur at a slow pace: Many generations may be needed for a noticeable change to arise in a species. This is indeed how Darwin understood the effect of natural selection, and he always referred to its cumulative effects over very many years. However, this needs not be the case and, in fact, selection may occur faster than the interaction between individuals (or of the individuals with their environment). Thus, recent experimental 
studies have reported observations of fast selection [38-40]. It is also conceivable that in man-monitored or laboratory processes one might make selection be the rapid influence rather than interaction. Another context where these ideas apply naturally is that of cultural evolution or social learning, where the time scale of selection is much closer to the time scale of interaction. Therefore, it is natural to ask about the consequences of the above assumption and the effect of relaxing it.

This issue has already been considered from an economic viewpoint in the context of equilibrium selection (but see an early biological example breaking the assumption of purely random matching in [41], which considered HawkDove games where strategists are more likely to encounter individuals using their same strategy). This refers to a situation in which for a game there is more than one equilibrium, like in the Stag Hunt game, given e.g. by the following payoff matrix

$$
\begin{gathered}
\text { C } \\
\text { C } \\
\text { D } \\
\left(\begin{array}{ll}
6 & 1 \\
5 & 2
\end{array}\right) .
\end{gathered}
$$

This game was already posed as a metaphor by Rousseau [42], which reads as follows: Two people go out hunting for stag, because two of them are necessary to hunt down such a big animal. However, any one of them can cheat the other by hunting hare, which one can do alone, leaving the other one in the impossibility of getting the stag. Therefore, we have a coordination game, in which the best option is to do as the other: Hunt stag together or both hunting hare separately. In game theory, this translates into the fact that both $\mathrm{C}$ and $\mathrm{D}$ are Nash equilibria, and in principle one is not able to determine which one would be selected by the players, i.e., which one is the solution of the game. One rationale to choose was proposed by Harsanyi and Selten ${ }^{2}$ [43], who classified C as the Pareto-efficient equilibrium (hunting stag is more profitable than hunting hare), because that is the most beneficial for both players, and D as the risk-dominant equilibrium, because it is the strategy that is better in case the other player chooses $\mathrm{D}$ (one can hunt hare alone). Here the tension arises then from the risk involved in cooperation, rather than from the temptation to defect of Snowdrift games [44] (note that both tensions are present in Prisoner's Dilemma).

Kandori et al. [45] showed that the risk-dominant equilibrium is selected when using a stochastic evolutionary game dynamics, proposed by Foster and Young [46], that considers that every player interacts with every other one (implying slow selection). However, fast selection leads to another result. Indeed, Robson and Vega-Redondo [47] considered the situation in which every player is matched to another one and therefore they only play one game before selection acts. In that case, they showed that the outcome changed and that the Pareto-efficient equilibrium is selected. This result was qualified later by Miekisz [48], who showed that the selected equilibrium depended on the population size and the mutation level of the dynamics. Recently, this issue has also been considered in [49], which compares the situation where the contribution of the game to the fitness is small (weak selection, see Section 4.6 below) to the one where the game is the main source of the fitness, finding that in the former the results are equivalent to the well-mixed population, but not in the latter, where the conclusions of [47] are recovered. It is also worth noticing in this regards the works by Boylan [50,51], where he studied the types of random matching that can still be approximated by continuous equations. In any case, even if the above are not general results and their application is mainly in economics, we have already a hint that time scales may play a non-trivial role in evolutionary games.

In fact, as we will show below, rapid selection affects evolutionary dynamics in such a dramatic way that for some games it even changes the stability of equilibria. We will begin our discussion by briefly summarizing results on a model for the emergence of altruistic behavior, in which the dynamics is not replicator-like, but that illustrates nicely the very important effects of fast selection. We will subsequently proceed to present a general theory for symmetric $2 \times 2$ games. There, in order to make explicit the relation between selection and interaction time scales, we use a discrete-time dynamics that produces results equivalent to the replicator dynamics when selection is slow. We will then show that the pace at which selection acts on the population is crucial for the appearance and stability of cooperation. Even in non-dilemma games such as the Harmony game [52], where cooperation is the only possible rational outcome, defectors may be selected for if population renewal is very rapid.

${ }^{2}$ Harsanyi and Senten received the Nobel Prize in Economics for this contribution, along with Nash, in 1994. 


\subsection{Time scales in the Ultimatum game}

As a first illustration of the importance of time scales in evolutionary game dynamics, we begin by dealing with this problem in the context of a specific set of such experiments, related to the Ultimatum game [53,54]. In this game, under conditions of anonymity, two players are shown a sum of money. One of the players, the "proposer", is instructed to offer any amount to the other, the "responder". The proposer can make only one offer, which the responder can accept or reject. If the offer is accepted, the money is shared accordingly; if rejected, both players receive nothing. Note that the Ultimatum game is not symmetric, in so far as proposer and responder have clearly different roles and are therefore not exchangeable. This will be our only such an example, and the remainder of the paper will only deal with symmetric games. Since the game is played only once (no repeated interactions) and anonymously (no reputation gain; for more on explanations of altruism relying on reputation see [55]), a self-interested responder will accept any amount of money offered. Therefore, self-interested proposers will offer the minimum possible amount, which will be accepted.

The above prediction, based on the rational character of the players, contrasts clearly with the results of actual Ultimatum game experiments with human subjects, in which average offers do not even approximate the self-interested prediction. Generally speaking, proposers offer respondents very substantial amounts (50\% being a typical modal offer) and respondents frequently reject offers below 30\% [56,57]. Most of the experiments have been carried out with university students in western countries, showing a large degree of individual variability but a striking uniformity between groups in average behavior. A large study in 15 small-scale societies [54] found that, in all cases, respondents or proposers behave in such a reciprocal manner. Furthermore, the behavioral variability across groups was much larger than previously observed: While mean offers in the case of university students are in the range $43-48 \%$, in the cross-cultural study they ranged from $26 \%$ to $58 \%$.

How does this fit in our focus topic, namely the emergence of cooperation? The fact that indirect reciprocity is excluded by the anonymity condition and that interactions are one-shot (repeated interaction, the mechanism proposed by Axelrod to foster cooperation [58,59], does not apply) allows one to interpret rejections in terms of the so-called strong reciprocity $[60,61]$. This amounts to considering that these behaviors are truly altruistic, i.e., that they are costly for the individual performing them in so far as they do not result in direct or indirect benefit. As a consequence, we return to our evolutionary puzzle: The negative effects of altruistic acts must decrease the altruist's fitness as compared to that of the recipients of the benefit, ultimately leading to the extinction of altruists. Indeed, standard evolutionary game theory arguments applied to the Ultimatum game lead to the expectation that, in a well-mixed population, punishers (individuals who reject low offers) have less chance to survive than rational players (individuals who accept any offer) and eventually disappear. We will now show that this conclusion depends on the dynamics, and that different dynamics may lead to the survival of punishers through fluctuations.

Consider a population of $N$ agents playing the Ultimatum game, with a fixed sum of money $M$ per game. Random pairs of players are chosen, of which one is the proposer and another one is the respondent. In its simplest version, we will assume that players are capable of other-regarding behavior (empathy); consequently, in order to optimize their gain, proposers offer the minimum amount of money that they would accept. Every agent has her own, fixed acceptance threshold, $1 \leqslant t_{i} \leqslant M$ ( $t_{i}$ are always integer numbers for simplicity). Agents have only one strategy: Respondents reject any offer smaller than their own acceptance threshold, and accept offers otherwise. Money shared as a consequence of accepted offers accumulates to the capital of each player, and is subsequently interpreted as fitness as usual. After $s$ games, the agent with the overall minimum fitness is removed (randomly picked if there are several) and a new agent is introduced by duplicating that with the maximum fitness, i.e., with the same threshold and the same fitness (again randomly picked if there are several). Mutation is introduced in the duplication process by allowing changes of \pm 1 in the acceptance threshold of the newly generated player with probability $1 / 3$ each. Agents have no memory (interactions are one-shot) and no information about other agents (no reputation gains are possible). We note that the dynamics of this model is not equivalent to the replicator equation, and therefore the results do not apply directly in that context. In fact, such an extremal dynamics leads to an amplification of the effect of fluctuations that allows to observe more clearly the influence of time scales. This is the reason why we believe it will help make our main point.

Fig. 1 shows the typical outcome of simulations of our model for a population of $N=1000$ individuals. An important point to note is that we are not plotting averages but a single realization for each value of $s$; the realizations we plot are not specially chosen but rather are representative of the typical simulation results. We have chosen to plot 

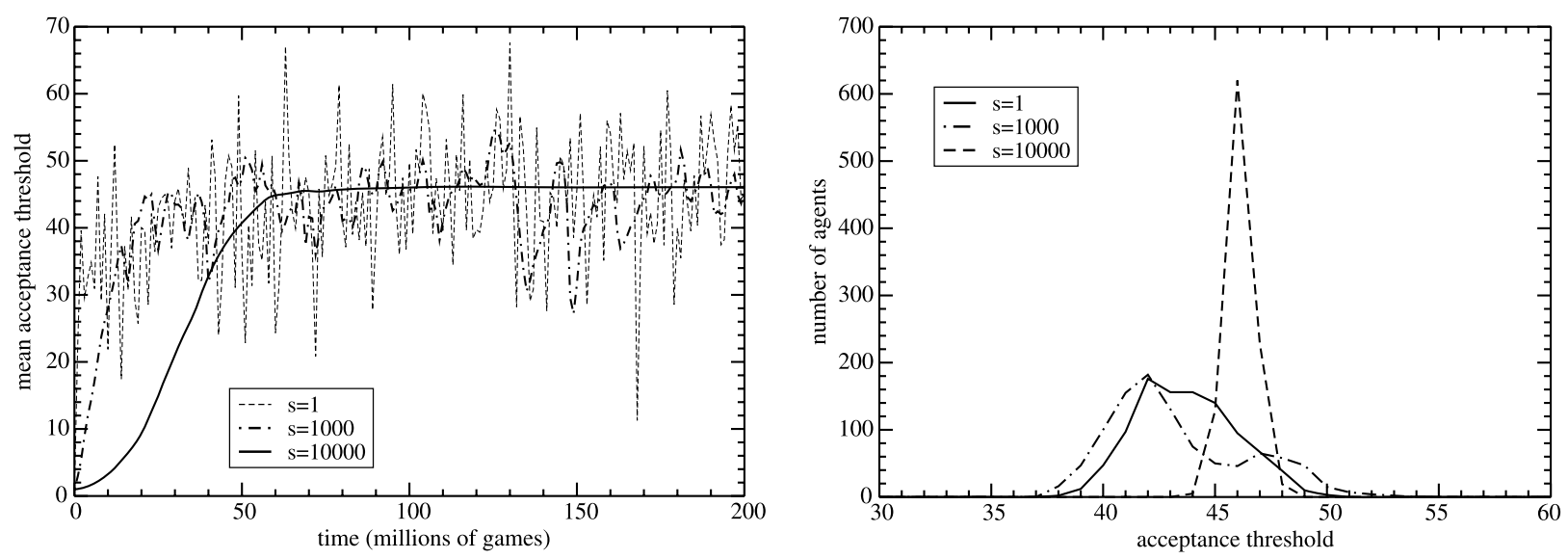

Fig. 1. Left: mean acceptance threshold as a function of simulation time. Initial condition is that all agents have $t_{i}=1$. Right: acceptance threshold distribution after $10^{8}$ games (note that this distribution, for small $s$, is not stationary). Initial condition is that all agents have uniformly distributed, random $t_{i}$. In both cases, $s$ is as indicated from the plot.

single realizations instead of averages to make clear for the reader the large fluctuations arising for small $s$, which are the key to understand the results and which we discuss below. As we can see, the mean acceptance threshold rapidly evolves towards values around $40 \%$, while the whole distribution of thresholds converges to a peaked function, with the range of acceptance thresholds for the agents covering about a $10 \%$ of the available ones. These are values compatible with the experimental results discussed above. The mean acceptance threshold fluctuates during the length of the simulation, never reaching a stationary value for the durations we have explored. The width of the peak fluctuates as well, but in a much smaller scale than the position. The fluctuations are larger for smaller values of $s$, and when $s$ becomes of the order of $N$ or larger, the evolution of the mean acceptance threshold is very smooth. As is clear from Fig. 1, for very small values of $s$, the differences in payoff arising from the fact that only some players play are amplified by our extreme dynamics, resulting in a very noisy behavior of the mean threshold. This is a crucial point and will be discussed in more detail below. Importantly, the typical evolution we are describing does not depend on the initial condition. In particular, a population consisting solely of self-interested agents, i.e. all initial thresholds set to $t_{i}=1$, evolves in the same fashion. Indeed, the distributions shown in the left panel of Fig. 1 (which again correspond to single realizations) have been obtained with such an initial condition, and it can be clearly observed that self-interested agents disappear in the early stages of the evolution. The number of players and the value $M$ of the capital at stake in every game are not important either, and increasing $M$ only leads to a higher resolution of the threshold distribution function, whereas smaller mutation rates simply change the pace of evolution.

To realize the effect of time scales, it is important to recall previous studies of the Ultimatum game by Page and Nowak $[62,63]$. The model introduced in those works has a dynamics completely different from ours: Following standard evolutionary game theory, every player plays with every other one in both roles (proponent and respondent), and afterwards players reproduce with probability proportional to their payoff (which is fitness in the reproductive sense). Simulations and adaptive dynamics equations show that the population ends up composed by players with fair $(50 \%)$ thresholds. Note that this is not what one would expect on a rational basis, but Page and Nowak traced this result back to empathy, i.e., the fact that the model is constrained to offer what one would accept. In any event, what we want to stress here is that their findings are also different from our observations: We only reach an equilibrium for large $s$. The reason for this difference is that the Page-Nowak model dynamics describes the $s / N \rightarrow \infty$ limit of our model, in which between death-reproduction events the time average gain obtained by all players is with high accuracy a constant $O(N)$ times the mean payoff. We thus see that our model is more general because it has one free parameter, $s$, that allows selecting different regimes whereas the Page-Nowak dynamics is only one limiting case. Those different regimes are what we have described as fluctuation dominated (when $s / N$ is finite and not too large) and the regime analyzed by Page and Nowak (when $s / N \rightarrow \infty$ ). This amounts to saying that by varying $s$ we can study regimes far from the standard evolutionary game theory limit. As a result, we find a variability of outcomes for the acceptance threshold consistent with the observations in real human societies [54,57]. Furthermore, if one considers that the acceptance threshold and the offer can be set independently, the results differ even more [64]: While 
in the model of Page and Nowak both magnitudes evolve to take very low values, close to zero, in the model presented here the results, when $s$ is small, are very similar to the one-threshold version, leading again to values compatible with the experimental observations. This in turn implies that rapid selection may be an alternative to empathy as an explanation of human behavior in this game.

The main message to be taken from this example is that fluctuations due to the finite number of games $s$ are very important. Among the results summarized above, the evolution of a population entirely formed by self-interested players into a diversified population with a large majority of altruists is the most relevant and surprising one. One can argue that the underlying reason for this is precisely the presence of fluctuations in our model. For the sake of definiteness, let us consider the case $s=1$ (agent replacement takes place after every game) although the discussion applies to larger (but finite) values of $s$ as well. After one or more games, a mutation event will take place and a "weak altruistic punisher" (an agent with $t_{i}=2$ ) will appear in the population, with a fitness inherited from its ancestor. For this new agent to be removed at the next iteration, our model rules imply that this agent has to have the lowest fitness, and also that it does not play as a proposer in the next game (if playing as a responder the agent will earn nothing because of her threshold). In any other event this altruistic punisher will survive at least one cycle, in which an additional one can appear by mutation. It is thus clear that fluctuations indeed help altruists to take over: As soon as a few altruists are present in the population, it is easy to see analytically that they will survive and proliferate even in the limit $s / N \rightarrow \infty$.

\subsection{Time scales in symmetric binary games}

The example in the previous subsection suggests that there certainly is an issue of relative time scales in evolutionary game theory that can have serious implications. In order to gain insight into this question, it is important to consider a general framework, and therefore we will now look at the general problem of symmetric $2 \times 2$ games. Asymmetric games can be treated similarly, albeit in a more cumbersome manner, and their classification involves many more types; we feel, therefore, that the symmetric case is a much clearer illustration of the effect of time scales. In what follows, we review and extend previous results of us $[65,66]$, emphasizing the consequences of the existence of different time scales.

Let us consider a population of $N$ individuals, each of whom plays with a fixed strategy, that can be either C or D (for "cooperate" and "defect" respectively, as in Section 2). We denote the payoff that an $X$-strategist gets when confronted to a $Y$-strategist ( $X$ and $Y$ are C or D) by the matrix element $W_{X Y}$. For a certain time individuals interact with other individuals in pairs randomly chosen from the population. During these interactions individuals collect payoffs. We shall refer to the interval between two interaction events as the interaction time. Once the interaction period has finished reproduction occurs, and in steady state selection acts immediately afterwards restoring the population size to the maximum allowed by the environment. The time between two of these reproduction/selection events will be referred to as the evolution time.

Reproduction and selection can be implemented in at least two different ways. The first one is through the FisherWright process [5] in which each individual generates a number of offspring proportional to her payoff. Selection acts by randomly killing individuals of the new generation until restoring the size of the population back to $N$ individuals. The second option for the evolution is the Moran process [5,67]. It amounts to randomly choosing an individual for reproduction proportionally to payoffs, whose single offspring replaces another randomly chosen individual, in this case with a probability $1 / N$ equal for all. In this manner populations always remains constant. The Fisher-Wright process is an appropriate model for species which produce a large number of offspring in the next generation but only a few of them survive, and the next generation replaces the previous one (like insects or some fishes). The Moran process is a better description for species which give rise to few offspring and reproduce in continuous time, because individuals neither reproduce nor die simultaneously, and death occurs at a constant rate. The original process was generalized to the frequency-dependent fitness context of evolutionary game theory by Taylor et al. [68], and used to study the conditions for selection favoring the invasion and/or fixation of new phenotypes. The results were found to depend on whether the population was infinite or finite, leading to a classification of the process in three or eight scenarios, respectively.

Both the Fisher-Wright and Moran processes define Markov chains $[69,70]$ on the population, characterized by the number of its C-strategists $n \in\{0,1, \ldots, N\}$, because in both cases it is assumed that the composition of the next generation is determined solely by the composition of the current generation. Each process defines a stochastic matrix 
$P$ with elements $P_{n, m}=p(m \mid n)$, the probability that the next generation has $m$ C-strategists provided the current one has $n$. While for the Fisher-Wright process all the elements of $P$ may be nonzero, for the Moran process the only nonzero elements are those for which $m=n$ or $m=n \pm 1$. Hence Moran is, in the jargon of Markov chains, a birth-death process with two absorbing states, $n=0$ and $n=N[69,70]$. Such a process is mathematically simpler, and for this reason it will be the one we will choose for our discussion on the effect of time scales.

To introduce explicitly time scales we will implement the Moran process in the following way, generalizing the proposal by Taylor et al. [68]. During $s$ time steps pairs of individuals will be chosen to play, one pair every time step. After that the above described reproduction/selection process will act according to the payoffs collected by players during the $s$ interaction steps. Then, the payoffs of all players are set to zero and a new cycle starts. Notice that in general players will play a different number of times - some not at all - and this will reflect in the collected payoffs. If $s$ is too small most players will not have the opportunity to play and chance will have a more prominent role in driving the evolution of the population.

Quantifying this effect requires that we first compute the probability that, in a population of $N$ individuals of which $n$ are C-strategists, an $X$-strategist is chosen to reproduce after the $s$ interaction steps. Let $n_{X Y}$ denote the number of pairs of $X$ - and $Y$-strategists that are chosen to play. The probability of forming a given pair, denoted $p_{X Y}$, will be

$$
p_{\mathrm{CC}}=\frac{n(n-1)}{N(N-1)}, \quad p_{\mathrm{CD}}=2 \frac{n(N-n)}{N(N-1)}, \quad p_{\mathrm{DD}}=\frac{(N-n)(N-n-1)}{N(N-1)} .
$$

Then the probability of a given set of $n_{X Y}$ is dictated by the multinomial distribution

$$
M\left(\left\{n_{X Y}\right\} ; s\right)= \begin{cases}s ! \frac{p_{\mathrm{CC}}^{n_{\mathrm{CC}}}}{n_{\mathrm{CC}} !} \frac{p_{\mathrm{CD}}^{n_{\mathrm{CD}}}}{n_{\mathrm{CD}} !} \frac{p_{\mathrm{DD}}^{n_{\mathrm{DD}}}}{n_{\mathrm{DD}} !}, & \text { if } n_{\mathrm{CC}}+n_{\mathrm{CD}}+n_{\mathrm{DD}}=s, \\ 0, & \text { otherwise. }\end{cases}
$$

For a given set of variables $n_{X Y}$, the payoffs collected by C- and D-strategists are

$$
W_{\mathrm{C}}=2 n_{\mathrm{CC}} W_{\mathrm{CC}}+n_{\mathrm{CD}} W_{\mathrm{CD}}, \quad W_{\mathrm{D}}=n_{\mathrm{CD}} W_{\mathrm{DC}}+2 n_{\mathrm{DD}} W_{\mathrm{DD}} .
$$

Then the probabilities of choosing a C- or D-strategist for reproduction are

$$
P_{\mathrm{C}}(n)=\mathrm{E}_{M}\left[\frac{W_{\mathrm{C}}}{W_{\mathrm{C}}+W_{\mathrm{D}}}\right], \quad P_{\mathrm{D}}(n)=\mathrm{E}_{M}\left[\frac{W_{\mathrm{D}}}{W_{\mathrm{C}}+W_{\mathrm{D}}}\right],
$$

where the expectations $\mathrm{E}_{M}[\cdot]$ are taken over the probability distribution $M(9)$. Notice that we have to guarantee $W_{X} \geqslant 0$ for the above expressions to define a true probability. This forces us to choose all payoffs $W_{X Y} \geqslant 0$. In addition, we have studied the effect of adding a baseline fitness to every player, which is equivalent to a translation of the payoff matrix $W$, obtaining the same qualitative results (see below).

Once these probabilities are obtained the Moran process accounts for the transition probabilities from a state with $n$ C-strategists to another with $n \pm 1 \mathrm{C}$-strategists. For $n \rightarrow n+1$ a C-strategist must be selected for reproduction (probability $\left.P_{\mathrm{C}}(n)\right)$ and a D-strategist for being replaced (probability $\left.(N-n) / N\right)$. Thus

$$
P_{n, n+1}=p(n+1 \mid n)=\frac{N-n}{N} P_{\mathrm{C}}(n) .
$$

For $n \rightarrow n-1$ a D-strategist must be selected for reproduction (probability $P_{\mathrm{D}}(n)$ ) and a C-strategist for being replaced (probability $n / N$ ). Thus

$$
P_{n, n-1}=p(n-1 \mid n)=\frac{n}{N} P_{\mathrm{D}}(n) .
$$

Finally, the transition probabilities are completed by

$$
P_{n, n}=1-P_{n, n-1}-P_{n, n+1} .
$$

\subsubsection{Slow selection limit}

Let us assume that $s \rightarrow \infty$, i.e. the evolution time is much longer than the interaction time. Then the distribution (9) will be peaked at the values $n_{X Y}=s p_{X Y}$, the larger $s$ the sharper the peak. Therefore in this limit

$$
P_{\mathrm{C}}(n) \rightarrow \frac{\overline{W_{\mathrm{C}}}(n)}{\overline{W_{\mathrm{C}}}(n)+\overline{W_{\mathrm{D}}}(n)}, \quad P_{\mathrm{D}}(n) \rightarrow \frac{\overline{W_{\mathrm{D}}}(n)}{\overline{\overline{W_{\mathrm{C}}}}(n)+\overline{W_{\mathrm{D}}}(n)},
$$


where

$$
\begin{aligned}
& \overline{W_{\mathrm{C}}}(n)=\frac{n}{N}\left[\frac{n-1}{N-1}\left(W_{\mathrm{CC}}-W_{\mathrm{CD}}\right)+W_{\mathrm{CD}}\right], \\
& \overline{W_{\mathrm{D}}}(n)=\frac{N-n}{N}\left[\frac{n}{N-1}\left(W_{\mathrm{DC}}-W_{\mathrm{DD}}\right)+W_{\mathrm{DD}}\right] .
\end{aligned}
$$

In general, for a given population size $N$ we have to resort to a numerical evaluation of the various quantities that characterize a birth-death process, according to the formulas in Appendix A. However, for large $N$ the transition probabilities can be expressed in terms of the fraction of C-strategists $x=n / N$ as

$$
\begin{aligned}
& P_{n, n+1}=x(1-x) \frac{w_{\mathrm{C}}(x)}{x w_{\mathrm{C}}(x)+(1-x) w_{\mathrm{D}}(x)}, \\
& P_{n, n-1}=x(1-x) \frac{w_{\mathrm{D}}(x)}{x w_{\mathrm{C}}(x)+(1-x) w_{\mathrm{D}}(x)},
\end{aligned}
$$

where

$$
w_{\mathrm{C}}(x)=x\left(W_{\mathrm{CC}}-W_{\mathrm{CD}}\right)+W_{\mathrm{CD}}, \quad w_{\mathrm{D}}(x)=x\left(W_{\mathrm{DC}}-W_{\mathrm{DD}}\right)+W_{\mathrm{DD}} .
$$

The terms $w_{\mathrm{C}}$ and $w_{\mathrm{D}}$ are, respectively, the expected payoff of a cooperator and a defector in this case of large $s$ and $N$. The factor $x(1-x)$ in front of $P_{n, n+1}$ and $P_{n, n-1}$ arises as a consequence of $n=0$ and $n=N$ being absorbing states of the process. There is another equilibrium $x^{*}$ where $P_{n, n \pm 1}=P_{n \pm 1, n}$, i.e. $w_{\mathrm{C}}\left(x^{*}\right)=w_{\mathrm{D}}\left(x^{*}\right)$, with $x^{*}$ given by

$$
x^{*}=\frac{W_{\mathrm{CD}}-W_{\mathrm{DD}}}{W_{\mathrm{DC}}-W_{\mathrm{CC}}+W_{\mathrm{CD}}-W_{\mathrm{DD}}} .
$$

For $x^{*}$ to be a valid equilibrium $0<x^{*}<1$ we must have

$$
\left(W_{\mathrm{DC}}-W_{\mathrm{CC}}\right)\left(W_{\mathrm{CD}}-W_{\mathrm{DD}}\right)>0 .
$$

This equilibrium is stable ${ }^{3}$ as long as the function $w_{\mathrm{C}}(x)-w_{\mathrm{D}}(x)$ is decreasing at $x^{*}$, for then if $x<x^{*} P_{n, n+1}>$ $P_{n+1, n}$ and if $x>x^{*} P_{n, n-1}>P_{n-1, n}$, i.e. the process will tend to restore the equilibrium, whereas if the function is increasing the process will be led out of $x^{*}$ by any fluctuation. In terms of (19) this implies

$$
W_{\mathrm{DC}}-W_{\mathrm{CC}}>W_{\mathrm{DD}}-W_{\mathrm{CD}}
$$

Notice that the two conditions

$$
w_{\mathrm{C}}\left(x^{*}\right)=w_{\mathrm{D}}\left(x^{*}\right), \quad w_{\mathrm{C}}^{\prime}\left(x^{*}\right)<w_{\mathrm{D}}^{\prime}\left(x^{*}\right),
$$

are precisely the conditions arising from the replicator dynamics for $x^{*}$ to be a stable equilibrium [10,11], albeit expressed in a different manner than in Section $2\left(w_{X}^{\prime}\right.$ represents the derivative of $w_{X}$ with respect to $\left.x\right)$. Out of the classic dilemmas, condition (21) holds for Stag Hunt and Snowdrift games, but condition (22) only holds for the latter. Thus, as we have already seen, only Snowdrift has a dynamically stable mixed population.

This analysis leads us to conclude that the standard setting of evolutionary games as advanced above, in which the time scale for reproduction/selection is implicitly (if not explicitly) assumed to be much longer than the interaction time scale, automatically yields the distribution of equilibria dictated by the replicator dynamics for that game. We have explicitly shown this to be true for binary games, but it can be extended to games with an arbitrary number of strategies. In the next section we will analyze what happens if this assumption on the time scales does not hold.

\footnotetext{
${ }^{3}$ Here the notion of stability implies that the process will remain near $x^{*}$ for an extremely long time, because as long as $N$ is finite, no matter how large, the process will eventually end up in $x=0$ or $x=1$, the absorbing states.
} 


\subsubsection{Fast selection limit}

When $s$ is finite, considering all the possible pairings and their payoffs, we arrive at

$$
\begin{aligned}
P_{\mathrm{C}}(n)= & \sum_{j=0}^{s} \sum_{k=0}^{s-j} 2^{s-j-k} \frac{s ! n^{s-k}(n-1)^{j}(N-n)^{s-j}(N-n-1)^{k}}{j ! k !(s-j-k) ! N^{s}(N-1)^{s}} \\
& \times \frac{2 j W_{\mathrm{CC}}+(s-j-k) W_{\mathrm{CD}}}{2 j W_{\mathrm{CC}}+2 k W_{\mathrm{DD}}+(s-j-k)\left(W_{\mathrm{CD}}+W_{\mathrm{DC}}\right)},
\end{aligned}
$$

and $P_{\mathrm{D}}(n)=1-P_{\mathrm{C}}(n)$. We have not been able to write this formula in a simpler way, so we have to evaluate it numerically for every choice of the payoff matrix. However, in order to have a glimpse at the effect of reducing the number of interactions between successive reproduction/selection events, we can examine analytically the extreme case $s=1$, for which

$$
\begin{aligned}
P_{n, n+1} & =\frac{n(N-n)}{N(N-1)}\left[\frac{2 W_{\mathrm{CD}}}{W_{\mathrm{DC}}+W_{\mathrm{CD}}}+\frac{n}{N} \frac{W_{\mathrm{DC}}-W_{\mathrm{CD}}}{W_{\mathrm{DC}}+W_{\mathrm{CD}}}-\frac{1}{N}\right], \\
P_{n, n-1} & =\frac{n(N-n)}{N(N-1)}\left[1+\frac{n}{N} \frac{W_{\mathrm{DC}}-W_{\mathrm{CD}}}{W_{\mathrm{DC}}+W_{\mathrm{CD}}}-\frac{1}{N}\right] .
\end{aligned}
$$

From these equations we find that

$$
\begin{aligned}
& \frac{P_{n, n-1}}{P_{n, n+1}}=\frac{D n+S(N-1)}{D(n+1)+S(N-1)-D(N+1)}, \\
& D=W_{\mathrm{DC}}-W_{\mathrm{CD}}, \quad S=W_{\mathrm{DC}}+W_{\mathrm{CD}},
\end{aligned}
$$

and this particular dependence on $n$ allows us to find the following closed-form expression for $c_{n}$, the probability that starting with $n$ cooperators the population ends up with all cooperators (see Appendix B)

$$
c_{n}=\frac{R_{n}}{R_{N}}, \quad R_{n}= \begin{cases}\prod_{j=1}^{n} \frac{S(N-1)+D j}{S(N-1)-D(N+1-j)}-1, & \text { if } D \neq 0, \\ n, & \text { if } D=0 .\end{cases}
$$

The first thing worth noticing in this expression is that it only depends on the two off-diagonal elements of the payoff matrix (through their sum, $S$, and difference, $D$ ). This means that in an extreme situation in which the evolution time is so short that it only allows a single pair of players to interact, the outcome of the game only depends on what happens when two players with different strategies play. The reason is obvious: Only those two players that have been chosen to play will have a chance to reproduce. If both players have strategy $X$, an $X$-strategist will be chosen to reproduce with probability 1 . Only if each player uses a different strategy the choice of the player that reproduces will depend on the payoffs, and in this case they are precisely $W_{\mathrm{CD}}$ and $W_{\mathrm{DC}}$. Of course, as $s$ increases this effect crosses over to recover the outcome for the case $s \rightarrow \infty$.

We can extend our analysis further for the case of large populations. If we denote $x=n / N$ and $c(x)=c_{n}$, then we can write, as $N \rightarrow \infty$,

$$
c(x) \sim \frac{e^{N \phi(x)}-1}{e^{N \phi(1)}-1}, \quad \phi(x)=\int_{0}^{x}[\ln (S+D t)-\ln (S+D(t-1))] d t .
$$

Then

$$
\phi^{\prime}(x)=\ln \left(\frac{S+D x}{S+D(x-1)}\right),
$$

which has the same sign as $D$, and hence $\phi(x)$ is increasing for $D>0$ and decreasing for $D<0$.

Thus if $D>0$, because of the factor $N$ in the argument of the exponentials and the fact that $\phi(x)>0$ for $x>0$, the exponential will increase sharply with $x$. Then, expanding around $x=1$,

$$
\phi(x) \approx \phi(1)-(1-x) \phi^{\prime}(1),
$$




$$
c(x) \sim \exp \{-N \ln (1+D / S)(1-x)\} .
$$

The outcome for this case is that absorption will take place at $n=0$ for almost any initial condition, except if we start very close to the absorbing state $n=N$, namely for $n \gtrsim N-1 / \ln (1+D / S)$.

On the contrary, if $D<0$ then $\phi(x)<0$ for $x>0$ and the exponential will be peaked at 0 . So expanding around $x=0$,

$$
\phi(x) \approx x \phi^{\prime}(0)
$$

and

$$
c(x) \sim 1-\exp \{-N \ln (1-D / S) x\} .
$$

The outcome in this case is therefore symmetrical with respect to the case $D>0$, because now the probability of ending up absorbed into $n=N$ is 1 for nearly all initial conditions except for a small range near $n=0$ determined by $n \lesssim 1 / \ln (1-D / S)$. In both cases the range of exceptional initial conditions increases with decreasing $|D|$, and in particular when $D=0$ the evolution becomes neutral, ${ }^{4}$ as it is reflected in the fact that in that special case $c_{n}=n / N$ (cf. Eq. (28)) [5].

In order to illustrate the effect of a finite $s$, even in the case when $s>1$, we will consider all possible symmetric $2 \times 2$ games. These were classified by Rapoport and Guyer [71] in 12 non-equivalent classes which, according to their Nash equilibria and their dynamical behavior under replicator dynamics, fall into three different categories:

(i) Six games have $W_{\mathrm{CC}}>W_{\mathrm{DC}}$ and $W_{\mathrm{CD}}>W_{\mathrm{DD}}$, or $W_{\mathrm{CC}}<W_{\mathrm{DC}}$ and $W_{\mathrm{CD}}<W_{\mathrm{DD}}$. For them, their unique Nash equilibrium corresponds to the dominant strategy ( $\mathrm{C}$ in the first case and $\mathrm{D}$ in the second case). This equilibrium is the global attractor of the replicator dynamics.

(ii) Three games have $W_{\mathrm{CC}}>W_{\mathrm{DC}}$ and $W_{\mathrm{CD}}<W_{\mathrm{DD}}$. They have several Nash equilibria, one of them with a mixed strategy, which is an unstable equilibrium of the replicator dynamics and therefore acts as a separator of the basins of attractions of two Nash equilibria in pure strategies, which are the attractors.

(iii) The remaining three games have $W_{\mathrm{CC}}<W_{\mathrm{DC}}$ and $W_{\mathrm{CD}}>W_{\mathrm{DD}}$. They also have several Nash equilibria, one of them with a mixed strategy, but in this case this is the global attractor of the replicator dynamics.

Examples of the first category are the Harmony and Prisoner's Dilemma games. Category (ii) includes the Stag Hunt game, whereas the Snowdrift game belongs to category (iii).

We will begin by considering one example of category (i): the Harmony game. To that aim we will choose the parameters $W_{\mathrm{CC}}=1, W_{\mathrm{CD}}=0.25, W_{\mathrm{DC}}=0.75$ and $W_{\mathrm{DD}}=0.01$. The name of this game refers to the fact that it represents no conflict, in the sense that all players get the maximum payoff by following strategy $\mathrm{C}$. The values of $c_{n}$ obtained for different populations $N$ and several values of $s$ are plotted in Fig. 2. The curves for large $s$ illustrate the no-conflicting character of this game as the probability $c_{n}$ is almost 1 for every starting initial fraction of C-strategists. The results for small $s$ also illustrate the effect of fast selection, as the inefficient strategy, D, is selected for almost any initial fraction of $\mathrm{C}$-strategists. The effect is more pronounced the larger the population. The crossover between the two regimes takes place at $s=2$ or 3, but it depends on the choice of payoffs. A look at Fig. 2 reveals that the crossing over to the $s \rightarrow \infty$ regime as $s$ increases has no connection whatsoever with $N$, because it occurs nearly at the same values for any population size $N$. It does depend, however, on the precise values of the payoffs. As a further check, in Fig. 3 we plot the results for $s=1$ for different population sizes $N$ and compare with the asymptotic prediction (32), showing its great accuracy for values of $N=100$ and higher; even for $N=10$ the deviation from the exact results is not large.

Let us now move to category (ii), well represented by the Stag Hunt game, discussed in the preceding subsection. We will choose for this game the payoffs $W_{\mathrm{CC}}=1, W_{\mathrm{CD}}=0.01, W_{\mathrm{DC}}=0.8$ and $W_{\mathrm{DD}}=0.2$. The values of $c_{n}$ obtained for different populations $N$ and several values of $s$ are plotted in Fig. 4. The panel (c) for $s=100$ reveals the behavior of the system according to the replicator dynamics: Both strategies are attractors, and the crossover fraction

${ }^{4}$ Notice that if $D=0$ then $W_{\mathrm{DC}}=W_{\mathrm{CD}}$ and therefore the evolution does not favor any of the two strategies. 


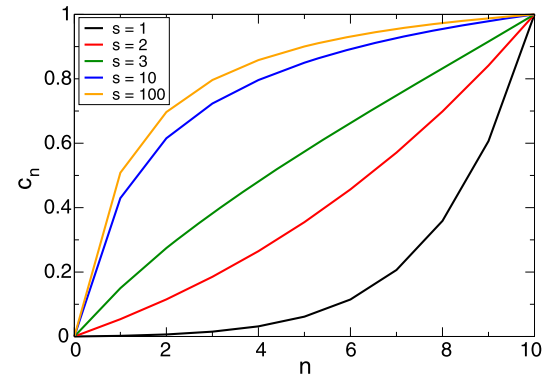

(a)

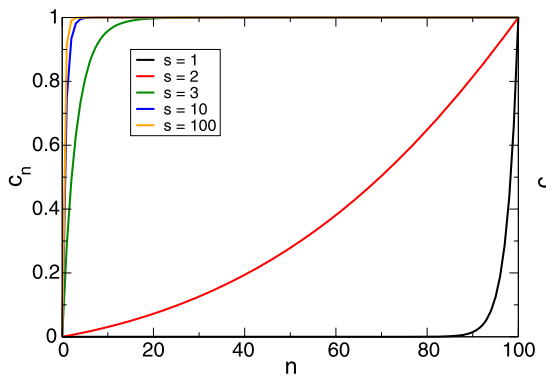

(b)

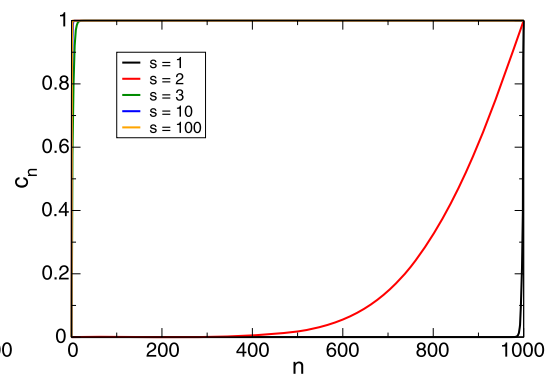

(c)

Fig. 2. Absorption probability $c_{n}$ to state $n=N$ starting from initial state $n$, for a Harmony game (payoffs $W_{\mathrm{CC}}=1, W_{\mathrm{CD}}=0.25, W_{\mathrm{DC}}=0.75$ and $W_{\mathrm{DD}}=0.01$ ), population sizes $N=10$ (a), $N=100$ (b) and $N=1000$ (c), and for values of $s=1,2,3,10$ and 100 . The values for $s=100$ are indistinguishable from the results of replicator dynamics.

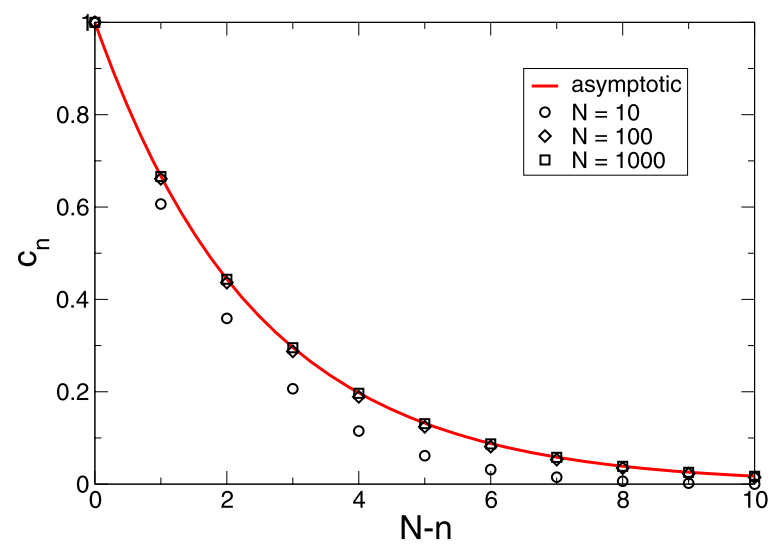

Fig. 3. Same as in Fig. 2 plotted against $N-n$, for $s=1$ and $N=10,100$ and 1000. The solid line is the asymptotic prediction (32).

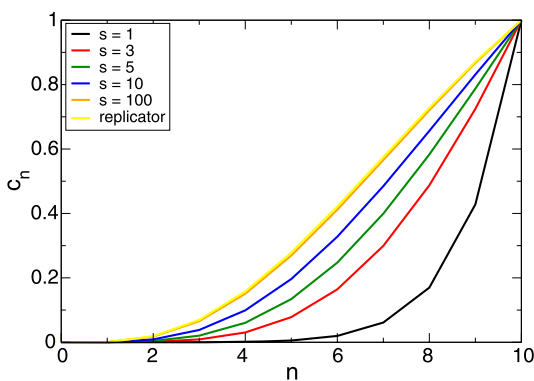

(a)

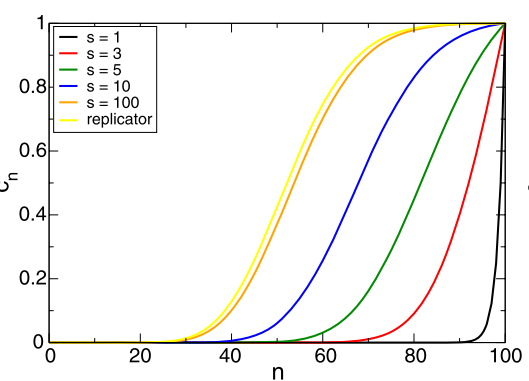

(b)

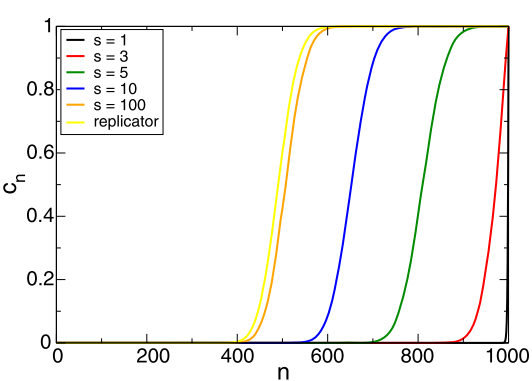

(c)

Fig. 4. Absorption probability $c_{n}$ to state $n=N$ starting from initial state $n$, for a Stag Hunt game (payoffs $W_{\mathrm{CC}}=1, W_{\mathrm{CD}}=0.01, W_{\mathrm{DC}}=0.8$ and $W_{\mathrm{DD}}=0.2$ ), population sizes $N=10$ (a), $N=100$ (b) and $N=1000$ (c), and for values of $s=1,3,5,10$ and 100 . Results from replicator dynamics are also plotted for comparison.

of C-strategists separating the two basins of attraction (given by Eq. (20)) is, for this case, $x^{*} \approx 0.49$. We can see that the effect of decreasing $s$ amounts to shifting this crossover towards 1, thus increasing the basins of attraction of the risk-dominated strategy. In the extreme case $s=1$ this strategy is the only attractor. Of course, for small population sizes (Fig. 4(a)) all these effects (the existence of the threshold and its shifting with decreasing $s$ ) are strongly softened, although still noticeable. An interesting feature of this game is that the effect of a finite $s$ is more persistent compared to what happens to the Harmony game. Whereas in the latter the replicator dynamics was practically recovered, for values of $s \geqslant 10$ we have to go up to $s=100$ to find the same in Stag Hunt. 


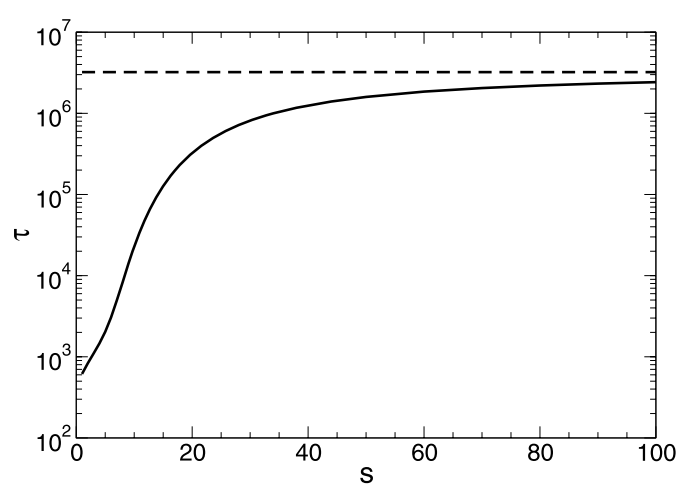

(a)

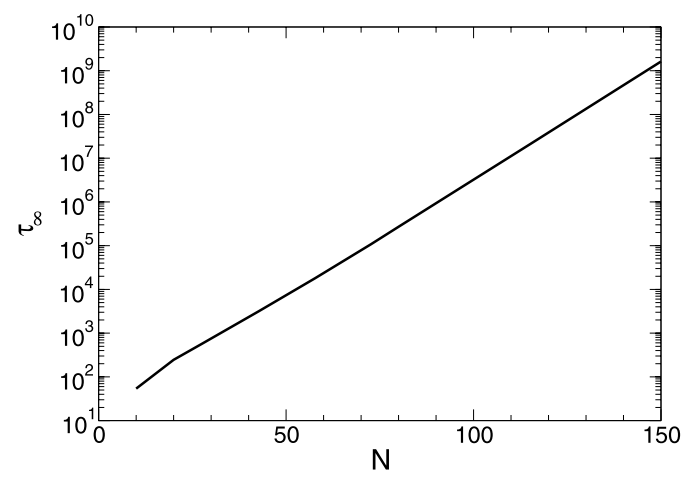

(b)

Fig. 5. Absorption time starting from the state $n / N=0.5$ for a Snowdrift game (payoffs $W_{\mathrm{CC}}=1, W_{\mathrm{CD}}=0.2, W_{\mathrm{DC}}=1.8$ and $W_{\mathrm{DD}}=0.01$ ), as a function of $s$ for population size $N=100$ (a) and as a function of $N$ in the limit $s \rightarrow \infty$ (b). Note the logarithmic scale for the absorption time.

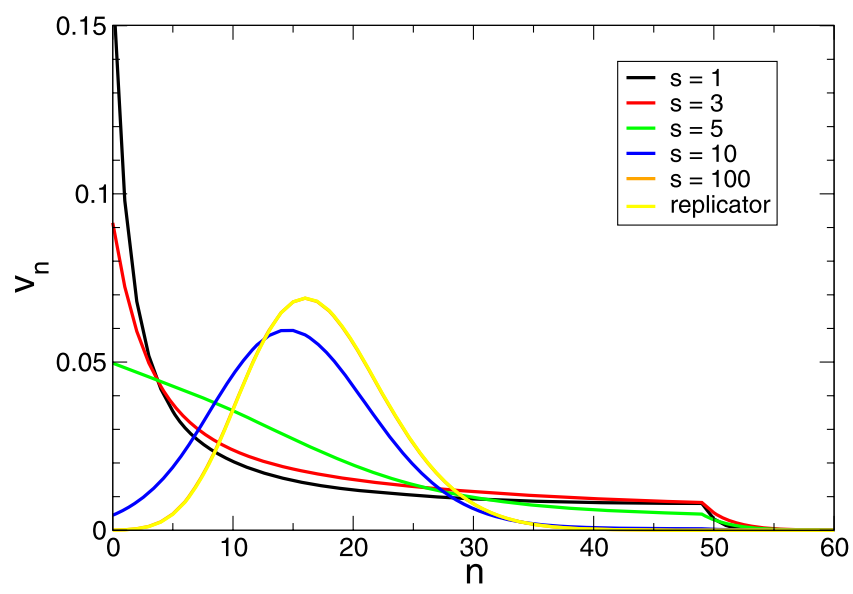

Fig. 6. Distribution of visits to state $n$ before absorption for population $N=100$, initial number of cooperators $n=50$ and several values of $s$. The game is the same Snowdrift game of Fig. 5. The curve for $s=100$ is indistinguishable from the one for $s \rightarrow \infty$ (labeled 'replicator').

Finally, a representative of category (iii) is the Snowdrift game, for which we will choose the payoffs $W_{\mathrm{CC}}=1$, $W_{\mathrm{CD}}=0.2, W_{\mathrm{DC}}=1.8$ and $W_{\mathrm{DD}}=0.01$. For these values, the replicator dynamics predicts that both strategies coexist with fractions of population given by $x^{*}$ in $(20)$, which for these parameters takes the value $x^{*} \approx 0.19$. However, a birth-death process for finite $N$ always ends up in absorption into one of the absorbing states. In fact, for any $s$ and $N$ and this choice of payoffs, the population always ends up absorbed into the $n=0$ state - except when it starts very close to $n=N$. But this case has a peculiarity that makes it entirely different from the previous ones. Whereas for the former cases the absorption time (50) is $\tau=O(N)$ regardless of the value of $s$, for Snowdrift the absorption time is $O(N)$ for $s=1$ but grows very fast with $s$ towards an asymptotic value $\tau_{\infty}$ (see Fig. 5(a)) and $\tau_{\infty}$ grows exponentially with $N$ (see Fig. 5(b)). This means that, while for $s=1$ the process behaves as in previous cases, being absorbed into the $n=0$ state, as $s$ increases there is a crossover to a regime in which the transient states become more relevant than the absorbing state because the population spends an extremely long time in them. In fact, the process oscillates around the mixed equilibrium predicted by the replicator dynamics. This is illustrated by the distribution of visits to states $0<n<N$ before absorption (48), shown in Fig. 6. Thus the effect of fast selection on Snowdrift games amounts to a qualitative change from the mixed equilibrium to the pure equilibrium at $n=0$.

Having illustrated the effect of fast selection in these three representative games, we can now present the general picture. Similar results arise in the remaining $2 \times 2$ games, fast selection favoring in all cases the strategy with the highest payoff against the opposite strategy. For the remaining five games of category (i) this means favoring the dominant strategy (Prisoner's Dilemma is a prominent example of it). The other two cases of category (ii) also 


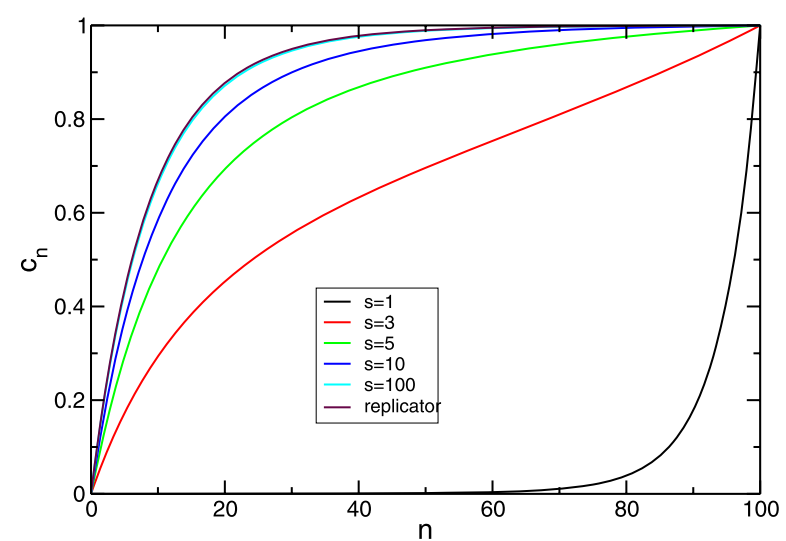

(a)

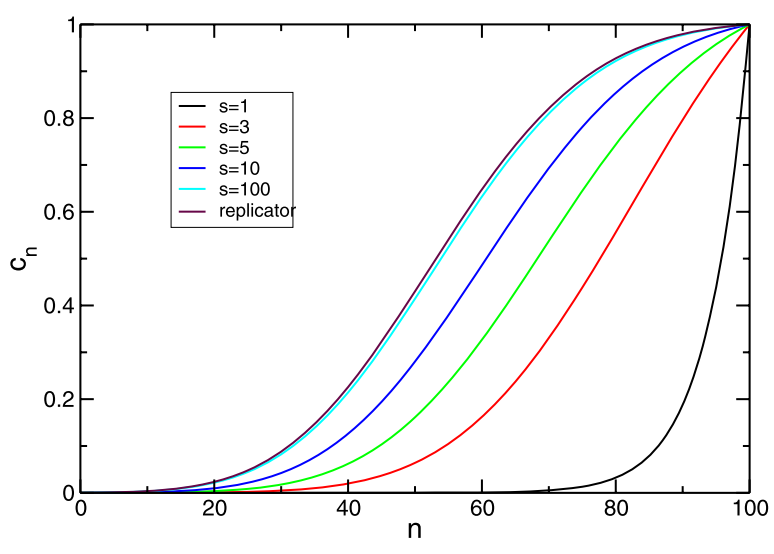

(b)

Fig. 7. Absorption probability starting from state $n$ for the Harmony game of Fig. 2 (a) and the Stag Hunt game of Fig. 4 (b) when $N=100$ and baseline fitness $f_{b}=1$.

experience a change in the basins of attraction of the two equilibria. Finally, the remaining two games of category (iii) experience the suppression of the coexistence state in favor of one of the two strategies. The conclusion of all this is that fast selection changes completely the outcome of replicator dynamics. In terms of cooperation, as the terms in the off-diagonal of social dilemmas verify $W_{\mathrm{DC}}>W_{\mathrm{CD}}$, this change in outcome has a negative influence on cooperation, as we have seen in all the games considered. Even for some payoff matrices of a non-dilemma game such as Harmony, it can make defectors invade the population.

Two final remarks are in order. First, these results do not change qualitatively with the population size. In fact, Eqs. (32) and (34) and Fig. 3 very clearly illustrate this. Second, there might be some concern about this analysis which the extreme $s=1$ case puts forward: All this might just be an effect of the fact that most players do not play and therefore have no chance to be selected for reproduction. In order to sort this out we have made a similar analysis but introducing a baseline fitness for all players, so that even if a player does not play she can still be selected for reproduction. The probability will be, of course, smaller than the one of the players who do play; however, we should bear in mind that when $s$ is very small, the overwhelming majority of players are of this type and this compensates for the smaller probability. Thus, let $f_{b}$ be the total baseline fitness that all players share per round, so that $s f_{b} / N$ is the baseline fitness every player has at the time reproduction/selection occurs. This choice implies that if $f_{b}=1$ the overall baseline fitness and that arising from the game are similar, regardless of $s$ and $N$. If $f_{b}$ is very small $\left(f_{b} \lesssim 0.1\right)$, the result is basically the same as that for $f_{b}=0$. The effect for $f_{b}=1$ is illustrated in Fig. 7 for Harmony and Stag Hunt games. Note also that at very large baseline fitness $\left(f_{b} \gtrsim 10\right)$ the evolution is almost neutral, although the small deviations induced by the game - which are determinant for the ultimate fate of the population - still follow the same pattern (see Fig. 8). Interestingly, Traulsen et al. [72] arrive at similar results by using a Fermi like rule (see Section 4.1 below) to introduce noise (temperature) in the selection process, and a interaction probability $q$ of interactions between individuals leading to heterogeneity in the payoffs, i.e., in the same words as above, to fluctuations, that in turn reduce the intensity of selection as is the case when we introduce a very large baseline fitness.

\section{Structured populations}

Having seen the highly non-trivial effects of considering temporal fluctuations in evolutionary games, in this section we are going to consider the effect of relaxing the well-mixed hypothesis by allowing the existence of spatial correlations in the population. Recall from Section 2.2 that a well-mixed population presupposes that every individual interacts with equal probability with every other one in the population, or equivalently that each individual interacts with the "average" individual. It is not clear, however, that this hypothesis holds in many practical situations. Territorial or physical constraints may limit the interactions between individuals, for example. On the other hand, an all-to-all network of relationships does not seem plausible in large societies; other key phenomena in social life, such as segregation or group formation, challenge the idea of a mean player that everyone interacts with. 


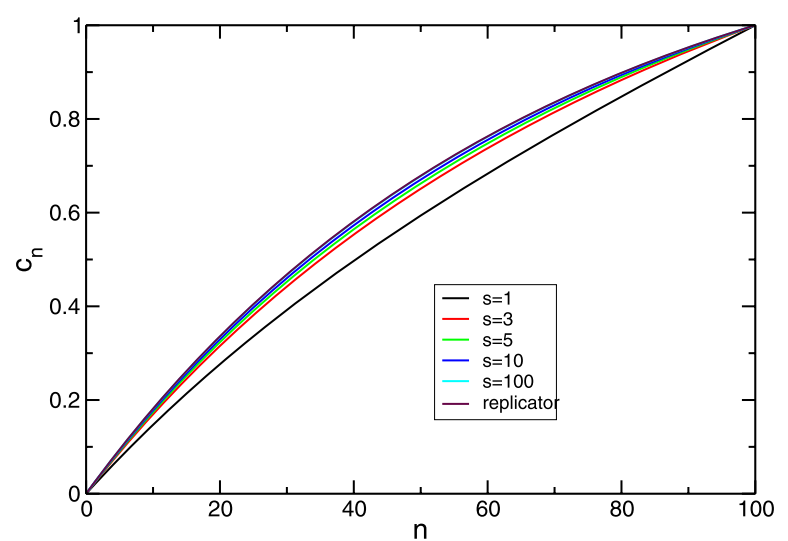

(a)

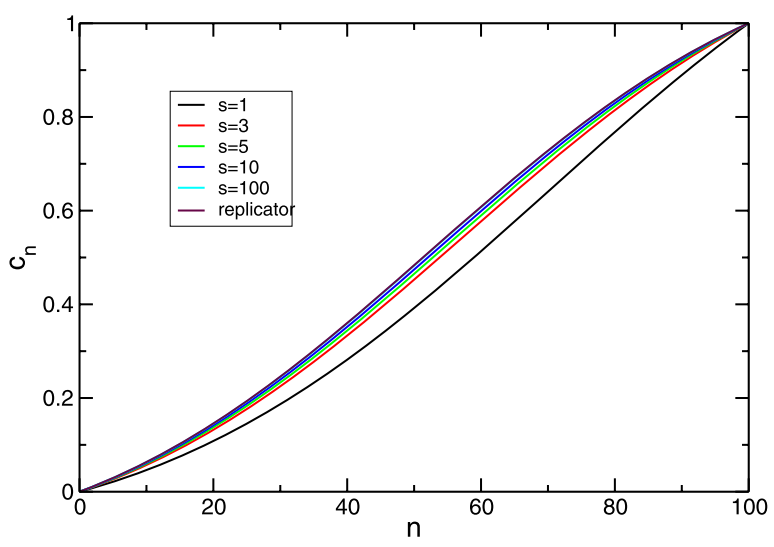

(b)

Fig. 8. Same as Fig. 7 for $f_{b}=10$.

It is adequate, therefore, to take into consideration the existence of a certain network of relationships in the population, which determines who interacts with whom. This network of relationships is what we will call from now on the structure of the population. Consistently, a well-mixed population will be labeled as unstructured and will be represented by a complete graph. Games on many different types of networks have been investigated, examples of which include regular lattices [73,74], scale-free networks [75], real social networks [76], etc. This section is not intended to be an exhaustive review of all this existent work and we refer the reader to [20] for such a detailed account. We rather want to give a panoramic and a more personal and idiosyncratic view of the field, based on the main available results and our own research.

It is at least reasonable to expect that the existence of structure in a population could give rise to the appearance of correlations and that they would have an impact on the evolutionary outcome. For more than fifteen years investigation into this phenomena has been a hot topic of research, as the seminal result by Nowak and May [73], which reported an impressive fostering of cooperation in Prisoner's Dilemma on spatial lattices, triggered a wealth of work focused on the extension of this effect to other games, networks and strategy spaces. On the other hand, the impossibility in most cases of analytical approaches and the complexity of the corresponding numerical agent-based models have made any attempt of exhaustive approach very demanding. Hence most studies have concentrated on concrete settings with a particular kind of game, which in most cases has been the Prisoner's Dilemma [73,77-91]. Other games has been much less studied in what concerns the influence of population structure, as show the comparatively much smaller number of works about Snowdrift or Hawk-Dove games [92-97], or Stag Hunt games [98-100]. Moreover, comprehensive studies in the space of $2 \times 2$ games are very scarce [74,75]. As a result, many interesting features of population structure and its influence on evolutionary games have been reported in the literature, but the scope of these conclusions is rather limited to particular models, so a general understanding of these issues, in the broader context of $2 \times 2$ games and different update rules, is generally missing.

However, the availability and performance of computational resources in recent years have allowed us to undertake a systematic and exhaustive simulation program [101,102] on these evolutionary models. As a result of this study we have reached a number of conclusions that are obviously in relation with previous research and that we will discuss in the following. In some cases, these are generalizations of known results to wider sets of games and update rules, as for example for the issue of the synchrony of the updating of strategies $[73,77,78,95,96,100,103]$ or the effect of small-world networks vs regular lattices $[84,96,104,105]$. In other cases, the more general view of our analysis has allowed us to integrate apparently contradictory results in the literature, as the cooperation on Prisoner's Dilemma vs. Snowdrift games $[73,92-94,96]$, or the importance of clustering in spatial lattices $[85,89,96]$. Other conclusions of ours, however, refute what seems to be established opinions in the field, as the alleged robustness of the positive influence of spatial structure on Prisoner's Dilemma [73,74,77]. And finally, we have reached novel conclusions that have not been highlighted by previous research, as the robustness of the influence of spatial structure on coordination games, or the asymmetry between the effects on games with mixed equilibria (coordination and anti-coordination games) and how it varies with the intensity of selection. 
It is important to make clear from the beginning that evolutionary games on networks may be sensitive to another source of variation with respect to replicator dynamics besides the introduction of spatial correlations. This source is the update rule, i.e. the rule that defines the evolution dynamics of individuals' strategies, whose influence seems to have been overlooked [74]. Strictly speaking, only when the model implements the so-called replicator rule (see below) one is considering the effect of the restriction of relationships that the population structure implies, in comparison with standard replicator dynamics. When using a different update rule, however, we are adding a second dimension of variability, which amounts to relax another assumption of replicator dynamics, namely number 4, which posits a population variation linear in the difference of payoffs (see Section 2). We will show extensively that this issue may have a huge impact on the evolutionary outcome.

In fact, we will see that there is not a general influence of population structure on evolutionary games. Even for a particular type of network, its influence on cooperation depends largely on the kind of game and the specific update rule. All one can do is to identify relevant topological characteristics that have a consistent effect on a broad range of games and update rules, and explain this influence in terms of the same basic principles. To this end, we will be looking at the asymptotic states for different values of the game parameters, and not at how the system behaves when the parameters are varied, which would be an approach of a more statistical mechanics character. In this respect, it is worth pointing out that some studies did use this perspective: thus, it has been shown that the extinction transitions when the temptation parameter varies within the Prisoner's Dilemma game and the evolutionary dynamics is stochastic fall in the directed percolation universality class, in agreement with a well known conjecture [106]. In particular, some of the pioneering works in using a physics viewpoint on evolutionary games $[82,107]$ have verified this result for specific models. The behavior changes under deterministic rules such as unconditional imitation (see below), for which this extinction transition is discontinuous.

Although our ultimate interest may be the effect on the evolution of cooperation, measuring to which extent cooperation is enforced or inhibited is not enough to clarify this effect. As in previous sections, our basic observables will be the dynamical equilibria of the model, in comparison with the equilibria of our reference model with standard replicator dynamics - which, as we have explained in Section 2, are closely related to those of the basic game. The understanding of how the population structure modifies qualitatively and quantitatively these equilibria will give us a much clearer view on the behavior and properties of the model under study, and hence on its influence on cooperation.

\subsection{Network models and update rules}

Many kinds of networks have been considered as models for population structure (for recent reviews on networks, see $[108,109])$. A first class includes networks that introduce a spatial arrangement of relationships, which can represent territorial or physical constraints in the interactions between individuals. Typical examples of this group are regular lattices, with different degrees of neighborhood. Other important group is that of synthetic networks that try to reproduce important properties that have been found in real networks, such as the small-world or scale-free properties. Prominent examples among these are Watts-Strogatz small-world networks [110] and Barabási-Albert scale-free networks [111]. Finally, "real" social networks that come directly from experimental data have also been studied, as for example in $[112,113]$.

As was stated before, one crucial component of the evolutionary models that we are discussing in this section is the update rule, which determines how the strategy of individuals evolves in time. There is a very large variety of update rules that have been used in the literature, each one arising from different backgrounds. The most important for our purposes is the replicator rule, also known as the proportional imitation rule, which is inspired on replicator dynamics and we describe in the following. ${ }^{5}$ Let $i=1 \ldots N$ label the individuals in the population. Let $s_{i}$ be the strategy of player $i, W_{i}$ her payoff and $N_{i}$ her neighborhood, with $k_{i}$ neighbors. One neighbor $j$ of player $i$ is chosen at random, $j \in N_{i}$. The probability of player $i$ adopting the strategy of player $j$ is given by

\footnotetext{
5 To our knowledge, Helbing was the first to show that a macroscopic population evolution following replicator dynamics could be induced by a microscopic imitative update rule [114,115]. Schlag proved later the optimality of such a rule under certain information constraints, and named it proportional imitation [116].
} 

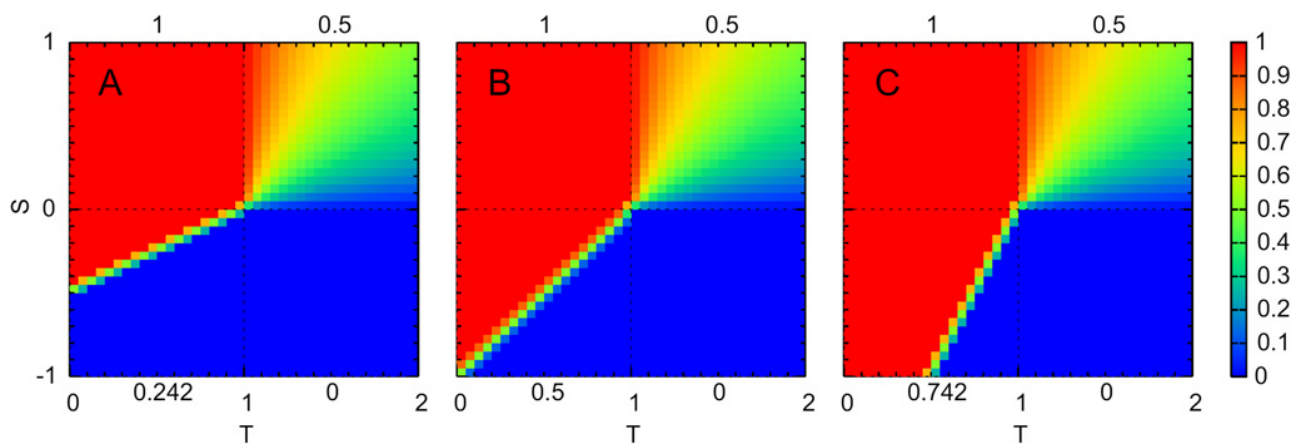

Fig. 9. Asymptotic density of cooperators $x^{*}$ with the replicator rule on a complete network, when the initial density of cooperators is $x^{0}=1 / 3$ (left, A), $x^{0}=1 / 2$ (middle, B) and $x^{0}=2 / 3$ (right, C). This is the standard outcome for a well-mixed population with replicator dynamics, and thus constitutes the reference to assess the influence of a given population structure (see main text for further details).

$$
p_{i j}^{t} \equiv \mathrm{P}\left\{s_{j}^{t} \rightarrow s_{i}^{t+1}\right\}= \begin{cases}\left(W_{j}^{t}-W_{i}^{t}\right) / \Phi, & W_{j}^{t}>W_{i}^{t}, \\ 0, & W_{j}^{t} \leqslant W_{i}^{t},\end{cases}
$$

with $\Phi$ appropriately chosen as a function of the payoffs to ensure $\mathrm{P}\{\cdot\} \in[0,1]$.

The reason for the name of this rule is the fact that the equation of evolution with this update rule, for large sizes of the population, is equal, up to a time scale factor, to that of replicator dynamics $[9,11]$. Therefore, the complete network with the replicator rule constitutes the finite-size, discrete-time version of replicator dynamics on an infinite, well-mixed population in continuous time. Fig. 9 shows the evolutionary outcome of this model, in the same type of plot as subsequent results in this section. Each panel of this figure displays the asymptotic density of cooperators $x^{*}$ for a different initial density $x^{0}$, in a grid of points in the $S T$-plane of games. The payoff matrix of each game is given by

$$
\begin{aligned}
& \text { C D } \\
& \text { C }\left(\begin{array}{ll}
1 & S \\
T & 0
\end{array}\right) \text {. }
\end{aligned}
$$

We will consider the generality of this choice of parameters at the end of this section, after introducing the other evolutionary rules. Note that, in the notation of Section 3, we have taken $W_{\mathrm{CC}}=1, W_{\mathrm{CD}}=S, W_{\mathrm{DC}}=T$, $W_{\mathrm{DD}}=0$; note also that for these payoffs, the normalizing factor in the replicator rule can be chosen as $\Phi=$ $\max \left(k_{i}, k_{j}\right)(\max (1, T)-\min (0, S))$. In this manner, we visualize the space of symmetric $2 \times 2$ games as a plane of co-ordinates $S$ and $T$ - for Sucker's and Temptation, which are the respective payoffs of a cooperator and a defector when confronting each other. The four quadrants represented correspond to the following games: Harmony (upper left), Stag Hunt (lower left), Snowdrift or Hawk-Dove (upper right) and Prisoner's Dilemma (lower right). As expected, these results reflect the close relationship between the equilibria of replicator dynamics and the equilibria of the basic game. Thus, all Harmony games end up in full cooperation and all Prisoner's Dilemmas in full defection, regardless of the initial condition. Snowdrift games reach a mixed strategy equilibrium, with density of cooperators $x_{e}=S /(S+T-1)$. Stag Hunt games are the only ones whose outcome depends on the initial condition, because of their bistable character with an unstable equilibrium also given by $x_{e}$. To allow a quantitative comparison of the degree of cooperation in each game, we have introduced a quantitative index, the average cooperation over the region corresponding to each game, which appears beside each quadrant. The results in Fig. 9 constitute the reference against which the effect of population structure will be assessed in the following.

One interesting variation of the replicator rule is the multiple replicator rule, whose difference consists on checking simultaneously all the neighborhood and thus making more probable a strategy change. With this rule the probability that player $i$ maintains her strategy is

$$
\mathrm{P}\left\{s_{i}^{t} \rightarrow s_{i}^{t+1}\right\}=\prod_{j \in N_{i}}\left(1-p_{i j}^{t}\right),
$$

with $p_{i j}^{t}$ given by (35). In case the strategy update takes place, the neighbor $j$ whose strategy is adopted by player $i$ is selected with probability proportional to $p_{i j}^{t}$. 
A different option is the following Moran-like rule, also called Death-Birth rule, inspired on the Moran dynamics, described in Section 3. With this rule a player chooses the strategy of one of her neighbors, or herself's, with a probability proportional to the payoffs

$$
\mathrm{P}\left\{s_{j}^{t} \rightarrow s_{i}^{t+1}\right\}=\frac{W_{j}^{t}-\Psi}{\sum_{k \in N_{i}^{*}}\left(W_{k}^{t}-\Psi\right)},
$$

with $N_{i}^{*}=N_{i} \cup\{i\}$. Because payoffs may be negative in Prisoner's Dilemma and Stag Hunt games, the constant $\Psi=\max _{j \in N_{i}^{*}}\left(k_{j}\right) \min (0, S)$ is subtracted from them. Note that with this rule a player can adopt, with low probability, the strategy of a neighbor that has done worse than herself.

The three update rules presented so far are imitative rules. Another important example of this kind is the unconditional imitation rule, also known as the "follow the best" rule [73]. With this rule each player chooses the strategy of the individual with largest payoff in her neighborhood, provided this payoff is greater than her own. A crucial difference with the previous rules is that this one is a deterministic rule.

Another rule that has received a lot of attention in the literature, specially in economics, is the best response rule. In this case, instead of some kind of imitation of neighbor's strategies based on payoff scoring, the player has enough cognitive abilities to realize whether she is playing an optimum strategy (i.e. a best response) given the current configuration of her neighbors. If it is not the case, she adopts with probability $p$ that optimum strategy. It is clear that this rule is innovative, as it is able to introduce strategies not present in the population, in contrast with the previous purely imitative rules.

Finally, an update rule that has been widely used in the literature, because of being analytically tractable, is the Fermi rule, based on the Fermi distribution function [82,117,118]. With this rule, a neighbor $j$ of player $i$ is selected at random (as with the replicator rule) and the probability of player $i$ acquiring the strategy of $j$ is given by

$$
\mathrm{P}\left\{s_{j}^{t} \rightarrow s_{i}^{t+1}\right\}=\frac{1}{1+\exp \left(-\beta\left(W_{j}^{t}-W_{i}^{t}\right)\right)} .
$$

The parameter $\beta$ controls the intensity of selection, and can be understood as the inverse of temperature or noise in the update rule. Low $\beta$ represents high temperature or noise and, correspondingly, weak selection pressure. Whereas this rule has been employed to study resonance-like behavior in evolutionary games on lattices [119], we use it in this work to deal with the issue of the intensity of selection (see Section 4.6).

Having introduced the evolutionary rules we will consider, it is important to recall our choice for the payoff matrix (36), and discuss its generality. Most of the rules (namely the replicator, the multiple replicator, the unconditional imitation and the best response rules) are invariant on homogeneous networks ${ }^{6}$ under translation and (positive) scaling of the payoff matrix. Among the remaining rules, the dynamics changes upon translation for the Moran rule and upon scaling for the Fermi rule. The corresponding changes in these last two cases amount to a modification of the intensity of selection, which we also treat in this work. Therefore, we consider that the parameterization of (36) is general enough for our purposes.

It is also important to realize that for a complete network, i.e. for a well-mixed or unstructured population, the differences between update rules may be not relevant, as far as they do not change in general the evolutionary outcome [121]. These differences, however, become crucial when the population has some structure, as we will point out in the following.

The results displayed in Fig. 9 have been obtained analytically, but the remaining results of this section come from the simulation of agent-based models. In all cases, the population size is $N=10^{4}$ and the allowed time for convergence is $10^{4}$ time steps, which we have checked it is enough to reach a stationary state. One time step represents one update event for every individual in the population, exactly in the case of synchronous update and on average in the asynchronous case, so it could be considered as one generation. The asymptotic density of cooperators is obtained averaging over the last $10^{3}$ time steps, and the values presented in the plots are the result of averaging over 100 realizations. Cooperators and defectors are randomly located at the beginning of evolution and, when applicable, networks have been built with periodic boundary conditions. See [101] for further details.

\footnotetext{
6 The invariance under translations of the payoff matrix does not hold if the network is heterogenous. In this case, players with higher degrees receive comparatively more (less) payoff under positive (negative) translations. Only very recently has this issue been studied in the literature [120].
} 


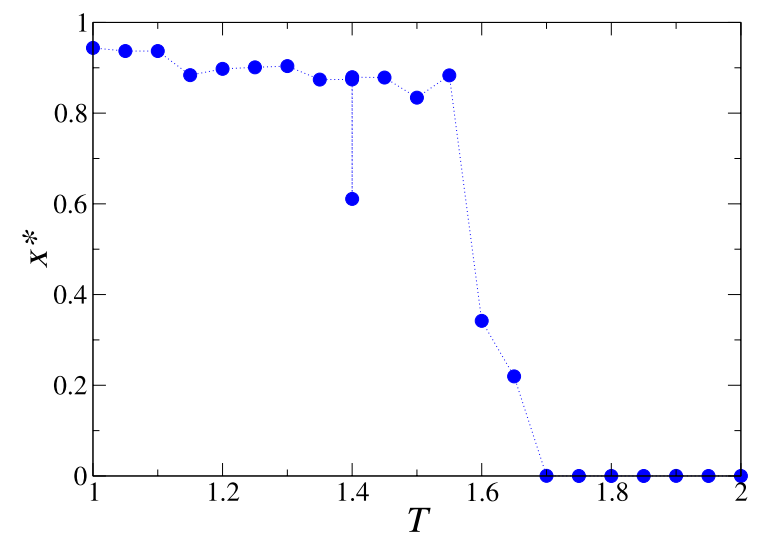

Fig. 10. Asymptotic density of cooperators $x^{*}$ in a square lattice with degree $k=8$ and initial density of cooperators $x^{0}=0.5$, when the game is the Prisoner's Dilemma as given by (40), proposed by Nowak and May [73]. Note that the outcome with replicator dynamics on a well-mixed population is $x^{*}=0$ for all the displayed range of the temptation parameter $T$. Notice also the singularity at $T=1.4$ with unconditional imitation. The surrounding points are located at $T=1.3999$ and $T=1.4001$.

\subsection{Spatial structure and homogeneous networks}

In 1992 Nowak and May published a very influential paper [73], where they showed the dramatic effect that the spatial distribution of a population could have on the evolution of cooperation. This has become the prototypical example of the promotion of cooperation favored by the structure of a population, also known as network reciprocity [19]. They considered the following Prisoner's Dilemma:

$$
\begin{gathered}
\mathrm{C} \\
\mathrm{C} \\
\mathrm{D}
\end{gathered}\left(\begin{array}{cc}
1 & 0 \\
T & \epsilon
\end{array}\right),
$$

with $1 \leqslant T \leqslant 2$ and $\epsilon \lesssim 0$. Note that this one-dimensional parameterization corresponds in the $S T$-plane to a line very near the boundary with Snowdrift games.

Fig. 10 shows the great fostering of cooperation reported by [73]. The authors explained this influence in terms of the formation of clusters of cooperators, which give cooperators enough payoff to survive even when surrounded by some defectors. This model has a crucial detail, whose importance we will stress later: The update rule used is unconditional imitation.

Since the publication of this work many studies have investigated related models with different games and networks, reporting qualitatively consistent results [20]. However, Hauert and Doebeli published in 2004 another important result [93], which casted a shadow of doubt on the generality of the positive influence of spatial structure on cooperation. They studied the following parameterization of Snowdrift games:

$$
\begin{gathered}
\mathrm{C} \\
\mathrm{C} \\
\mathrm{D}
\end{gathered}\left(\begin{array}{cc}
1 & 2-T \\
T & 0
\end{array}\right),
$$

with $1 \leqslant T \leqslant 2$ again.

The unexpected result obtained by the authors is displayed in Fig. 11. Only for low $T$ there is some improvement of cooperation, whereas for medium and high $T$ cooperation is inhibited. This is a surprising result, because the basic game, the Snowdrift, is in principle more favorable to cooperation. As we have seen above, its only stable equilibrium is a mixed strategy population with some density of cooperators, whereas the unique equilibrium in Prisoner's Dilemma is full defection (see Fig. 9). In fact, a previous paper by Killingback and Doebeli [92] on the Hawk-Dove game, a game equivalent to the Snowdrift game, had reported an effect of spatial structure equivalent to a promotion of cooperation. 


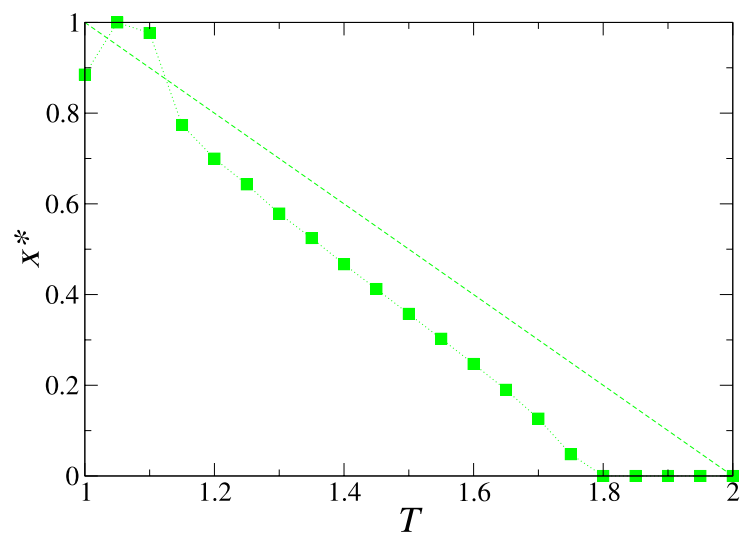

Fig. 11. Asymptotic density of cooperators $x^{*}$ in a square lattice with degree $k=8$ and initial density of cooperators $x^{0}=0.5$, when the game is the Snowdrift game as given by (41), proposed by Hauert and Doebeli [93]. The result for a well-mixed population is displayed as a reference as a dashed line.

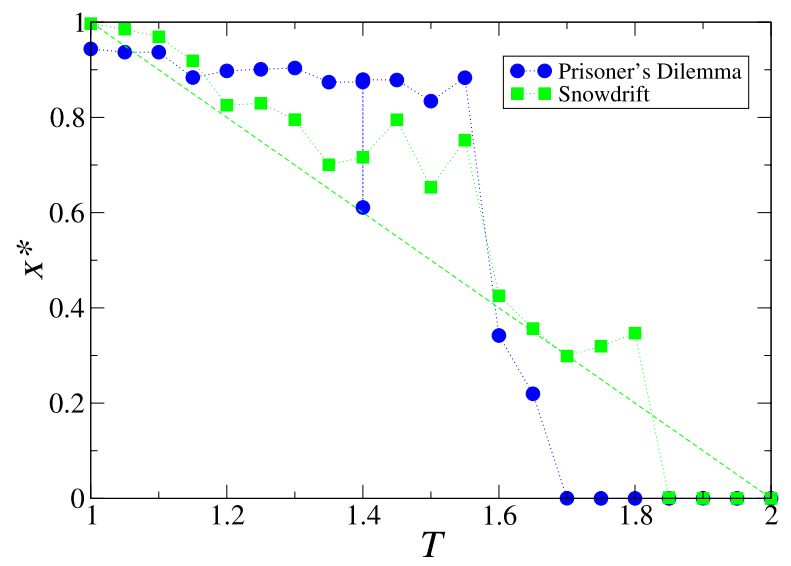

(a)

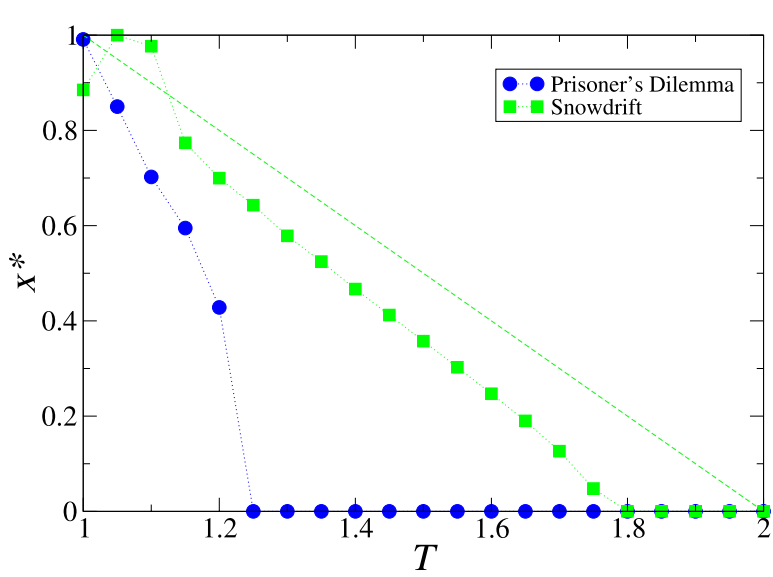

(b)

Fig. 12. Asymptotic density of cooperators $x^{*}$ in square lattices with degree $k=8$ and initial density of cooperators $x^{0}=0.5$, for both Prisoner's Dilemma (40) and Snowdrift games (41), displayed separately according to the update rule: (a) unconditional imitation (Nowak and May's model [73]), (b) replicator rule (Hauert and Doebeli's model [93]). The result for Snowdrift in a well-mixed population is displayed as a reference as a dashed line. It is clear the similar influence of regular lattices on both games, when the key role of the update rule is taken into account (see main text for details).

Hauert and Doebeli explained their result in terms of the hindrance to cluster formation and growth, at the microscopic level, caused by the payoff structure of the Snowdrift game. Notwithstanding the different cluster dynamics in both games, as observed by the authors, a hidden contradiction looms in their argument, because it implies some kind of discontinuity in the microscopic dynamics in the crossover between Prisoner's Dilemma and Snowdrift games $(S=0,1 \leqslant T \leqslant 2$ ). However, the equilibrium structure of both basic games, which drives this microscopic dynamics, is not discontinuous at this boundary, because for both games the only stable equilibrium is full defection. So, where does this change in the cluster dynamics come from?

The fact is that there is not such a difference in the cluster dynamics between Prisoner's Dilemma and Snowdrift games, but different update rules in the models. Nowak and May [73], and Killingback and Doebeli [92], used the unconditional imitation rule, whereas Hauert and Doebeli [93] employed the replicator rule. The crucial role of the update rule becomes clear in Fig. 12, where results in Prisoner's Dilemma and Snowdrift are depicted separately for each update rule. It shows that, if the update rule used in the model is the same, the influence on both games, in terms of promotion or inhibition of cooperation, has a similar dependence on $T$. For both update rules, cooperation is fostered in Prisoner's Dilemma and Snowdrift at low values of $T$, and cooperation is inhibited at high $T$. Note that 

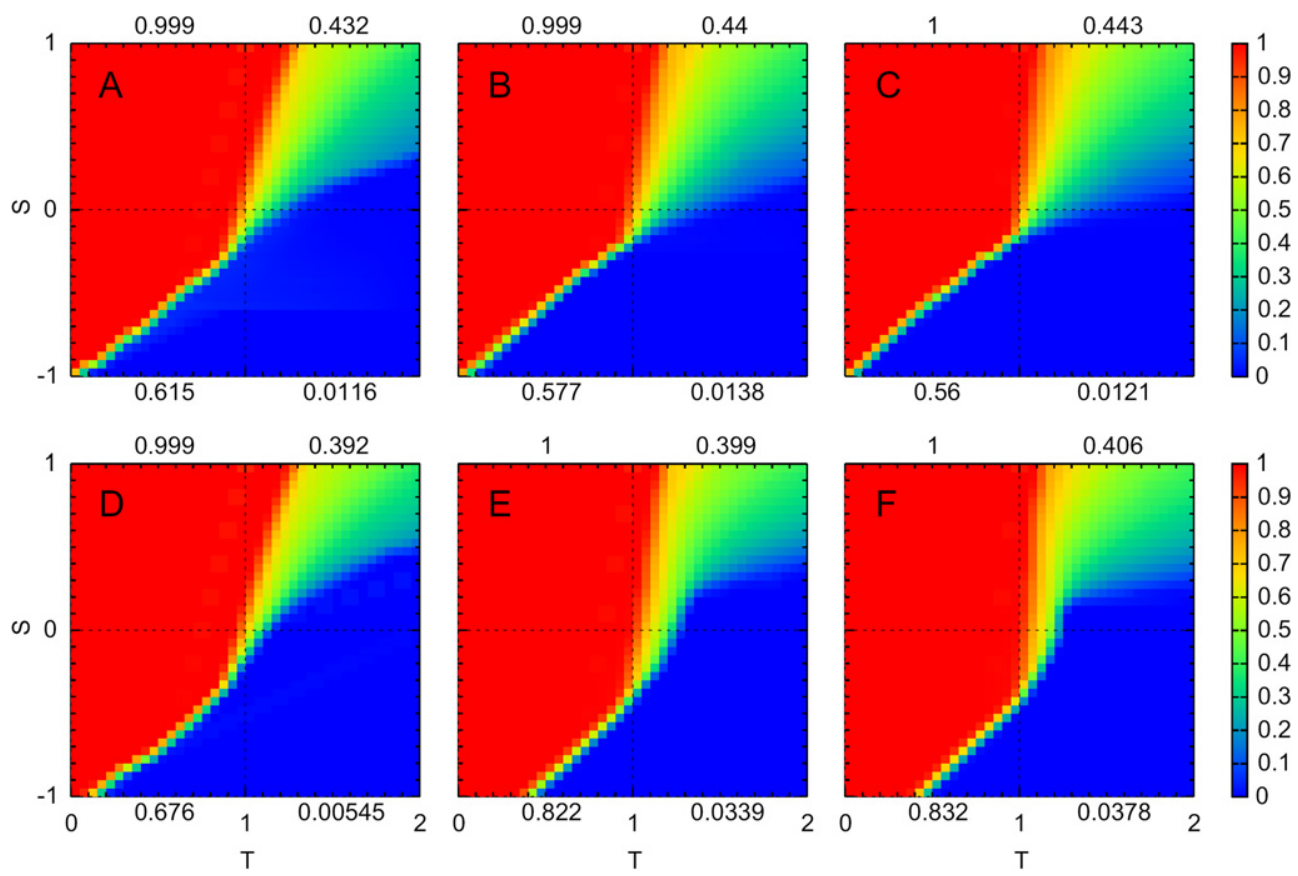

Fig. 13. Asymptotic density of cooperators $x^{*}$ in homogeneous random networks (upper row, A to C) compared to regular lattices (lower row, D to F), with degrees $k=4(\mathrm{~A}, \mathrm{D}), 6(\mathrm{~B}, \mathrm{E})$ and $8(\mathrm{C}, \mathrm{F})$. The update rule is the replicator rule and the initial density of cooperators is $x^{0}=0.5$. The plots show that the main influence occurs in Stag Hunt and Snowdrift games, specially for regular lattices with large clustering coefficient, $k=6$ and 8 (see main text).

with unconditional imitation the crossover between both behaviors takes place at $T \approx 1.7$, whereas with the replicator rule it occurs at a much lower value of $T \approx 1.15$. The logic behind this influence is better explained in the context of the full $S T$-plane, as we will show later.

The fact that this apparent contradiction has been resolved considering the role of the update rule is a good example of its importance. This conclusion is in agreement with those of [96], which performed an exhaustive study on Snowdrift games with different network models and update rules, but refutes those of [74], which defended that the effect of spatial lattices was almost independent of the update rule. In consequence, the influence of the network models that we consider in the following is presented separately for each kind of update rule, highlighting the differences in results when appropriate. Apart from this, to assess and explain the influence of spatial structure, we need to consider it along with games that have different equilibrium structures, not only a particular game, in order to draw sufficiently general conclusions. One way to do it is to study their effect on the space of $2 \times 2$ games described by the parameters $S$ and $T$ (36). A first attempt was done by Hauert [74], but some problems in this study make it inconclusive (see [101] for details on this issue).

Apart from lattices of different degrees (4, 6 and 8), we have also considered homogeneous random networks, i.e. random networks where each node has exactly the same number of neighbors. The aim of comparing with this kind of networks is to isolate the effect of the spatial distribution of individuals from that of the mere limitation of the number of neighbors and the context preservation [85] of a degree-homogeneous random network. The well-mixed population hypothesis implies that every player plays with the "average" player in the population. From the point of view of the replicator rule this means that every player samples successively the population in each evolution step. It is not unreasonable to think that if the number of neighbors is sufficiently restricted the result of this random sampling will differ from the population average, thus introducing changes in the evolutionary outcome.

Fig. 13 shows the results for the replicator rule with random and spatial networks of different degrees. First, it is clear that the influence of these networks is negligible on Harmony games and minimal on Prisoner's Dilemmas, given the reduced range of parameters where it is noticeable. There is, however, a clear influence on Stag Hunt and Snowdrift games, which is always of opposite sign: An enhancement of cooperation in Stag Hunt and an inhibition in Snowdrift. Second, it is illuminating to consider the effect of increasing the degree. For the random network, it means 
that its weak influence vanishes. The spatial lattice, however, whose result is very similar to that of the random one for the lowest degree $(k=4)$, displays remarkable differences for the greater degrees $(k=6$ and 8$)$. These differences are a clear promotion of cooperation in Stag Hunt games and a lesser, but measurable, inhibition in Snowdrift games, specially for low $S$.

The relevant topological feature that underlies this effect is the existence of clustering in the network, understood as the presence of triangles or, equivalently, common neighbors $[108,109]$. In regular lattices, for $k=4$ there is no clustering, but there is for $k=6$ and 8. This point explains the difference between the conclusions of Cohen et al. [85] and those of Ifti et al. [89] and Tomassini et al. [96], regarding the role of network clustering in the effect of spatial populations. In [85], rectangular lattices of degree $k=4$ were considered, which have strictly zero clustering because there are not closed triangles in the network, hence finding no differences in outcome between the spatial and the random topology. In the latter case, on the contrary, both studies employed rectangular lattices of degree $k=8$, which do have clustering, and thus they identified it as a key feature of the network, for particular parameterizations of the games they were studying, namely Prisoner's Dilemma [89] and Snowdrift [96].

An additional evidence for this conclusion is the fact that small-world networks, which include random links to reduce the average path between nodes while maintaining the clustering, produce almost indistinguishable results from those of Fig. 13 D-F. This conclusion is in agreement with existent theoretical work about small-world networks, on Prisoner's Dilemma [84,104,105] and its extensions [122,123], on Snowdrift games [96], and also with experimental studies on coordination games [124]. The difference between the effect of regular lattices and small-world networks consists, in general, in a greater efficiency of the latter in reaching the stationary state (see [101] for a further discussion on this comparison).

The mechanism that explains this effect is the formation and growth of clusters of cooperators, as Fig. 14 displays for a particular realization. The outcome of the population is then totally determined by the stability and growth of these clusters, which in turn depend on the dynamics of clusters interfaces. This means that the result is no longer determined by the global population densities but by the local densities that the players at the cluster interfaces see in their neighborhood. In fact, the primary effect that the network clustering causes is to favor, i.e., to maintain or to increase, the high local densities that were present in the population from the random beginning. This favoring produces opposite effects in Stag Hunt and Snowdrift games. As an illustrating example, consider that the global density is precisely that of the mixed equilibrium of the game. In Stag Hunt games, as this equilibrium is unstable, a higher local density induces the conversion of nearby defectors to cooperators, thus making the cluster grow. In Snowdrift games, on the contrary, as the equilibrium is stable, it causes the conversion of cooperators to defectors. See [101] for a full discussion on this mechanism.

In view of this, recalling that these are the results for the replicator rule, and that therefore they correspond to the correct update rule to study the influence of population structure on replicator dynamics, we can state that the presence of clustering (triangles, common neighbors) in a network is a relevant topological feature for the evolution of cooperation. Its main effects are, on the one hand, a promotion of cooperation in Stag Hunt games, and, on the other hand, an inhibition (of lower magnitude) in Snowdrift games. We note, however, that clustering may not be the only relevant factor governing the game asymptotics: one can devise peculiar graphs, not representing proper spatial structure, where other influences prove relevant. This is the case of networks consisting of a complete subgraphs connected to each other by a few connections [119], a system whose behavior, in spite of the high clustering coefficient, is similar to those observed on the traditional square lattice where the clustering coefficient is zero. This was subsequently related [125] to the existence of overlapping triangles that support the spreading of cooperation. We thus see that our claim about the outcome of evolutionary games on networks with clustering is anything but general and depends on the translational invariance of the network.

Other stochastic non-innovative rules, such as the multiple replicator and Moran rules, yield similar results, without qualitative differences [101]. Unconditional imitation, on the contrary, has a very different influence, as can be seen in Fig. 15.

In the first place, homogenous random networks themselves have a marked influence, that increases with network degree for Stag Hunt and Snowdrift games, but decreases for Prisoner's Dilemmas. Secondly, there are again no important differences between random and spatial networks if there is no clustering in the network (note how the transitions between the different regions in the results are the same). There are, however, stark differences when there is clustering in the network. Interestingly, these are the cases with an important promotion of cooperation in Snowdrift and Prisoner's Dilemma games. 

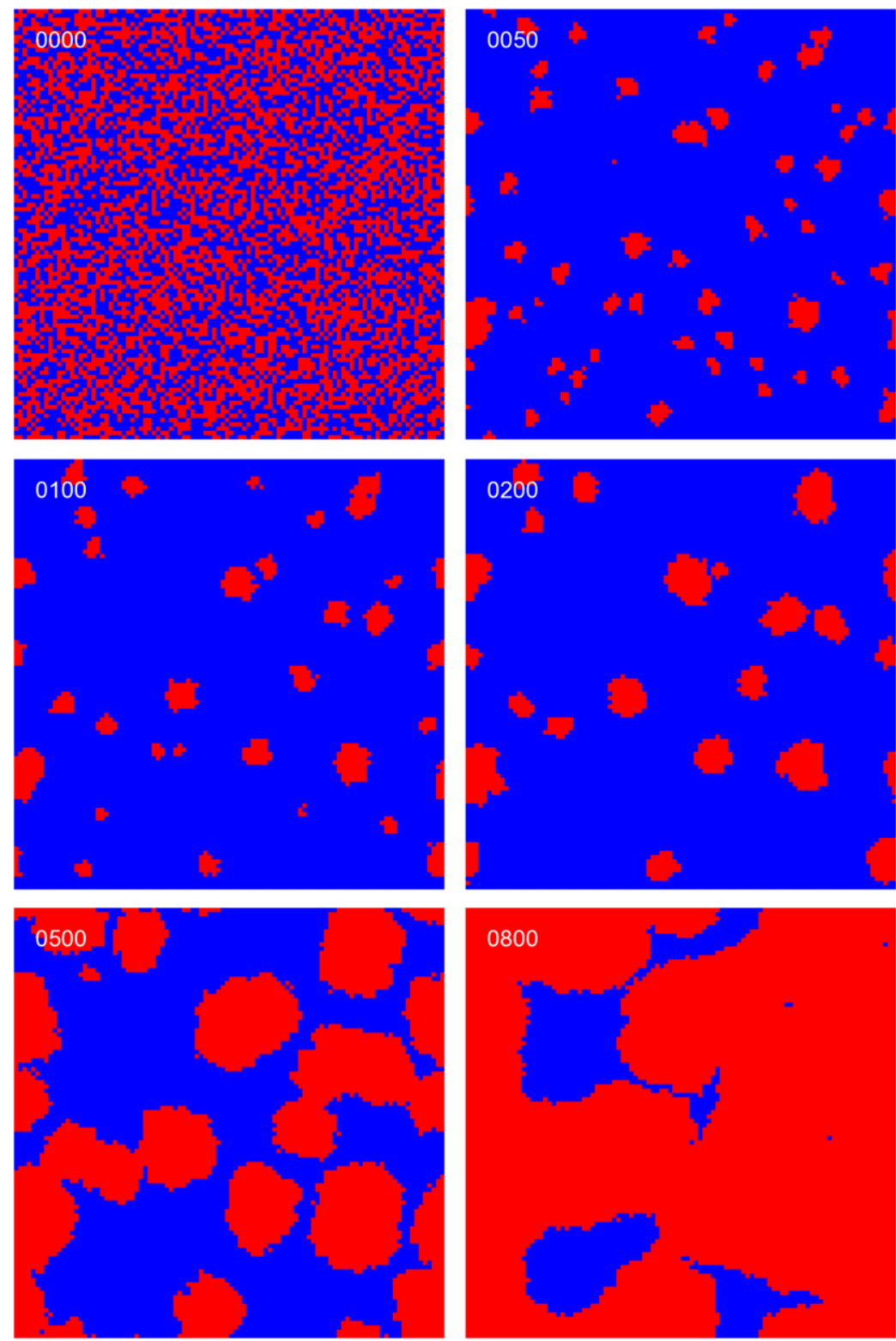

Fig. 14. Snapshots of the evolution of a population on a regular lattice of degree $k=8$, playing a Stag Hunt game $(S=-0.65$ and $T=0.65)$. Cooperators are displayed in red and defectors in blue. The update rule is the replicator rule and the initial density of cooperators is $x^{0}=0.5$. The upper left label shows the time step $t$. During the initial steps, cooperators with low local density of cooperators in their neighborhood disappear, whereas those with high local density grow into the clusters that eventually take up the complete population. (For interpretation of the references to color in this figure legend, the reader is referred to the web version of this article.)

In this case, the dynamical mechanism is the formation and growth of clusters of cooperators as well, and the fate of the population is again determined by the dynamics of cluster interfaces. With unconditional imitation, however, given its deterministic nature, interfaces advance one link every time step. This makes very easy the calculation of the conditions for their advancement, because these conditions come down to those of a flat interface between cooperators and defectors [101]. See Fig. 16 for a typical example of evolution.

An interesting consequence of the predominant role of flat interfaces with unconditional imitation is that, as long as there is in the initial population a flat interface (i.e., a cluster with it, as for example a $3 \times 2$ cluster in a 8 -neighbor lattice), the cluster will grow and eventually extend to the entire population. This feature corresponds to the $3 \times 3$ 

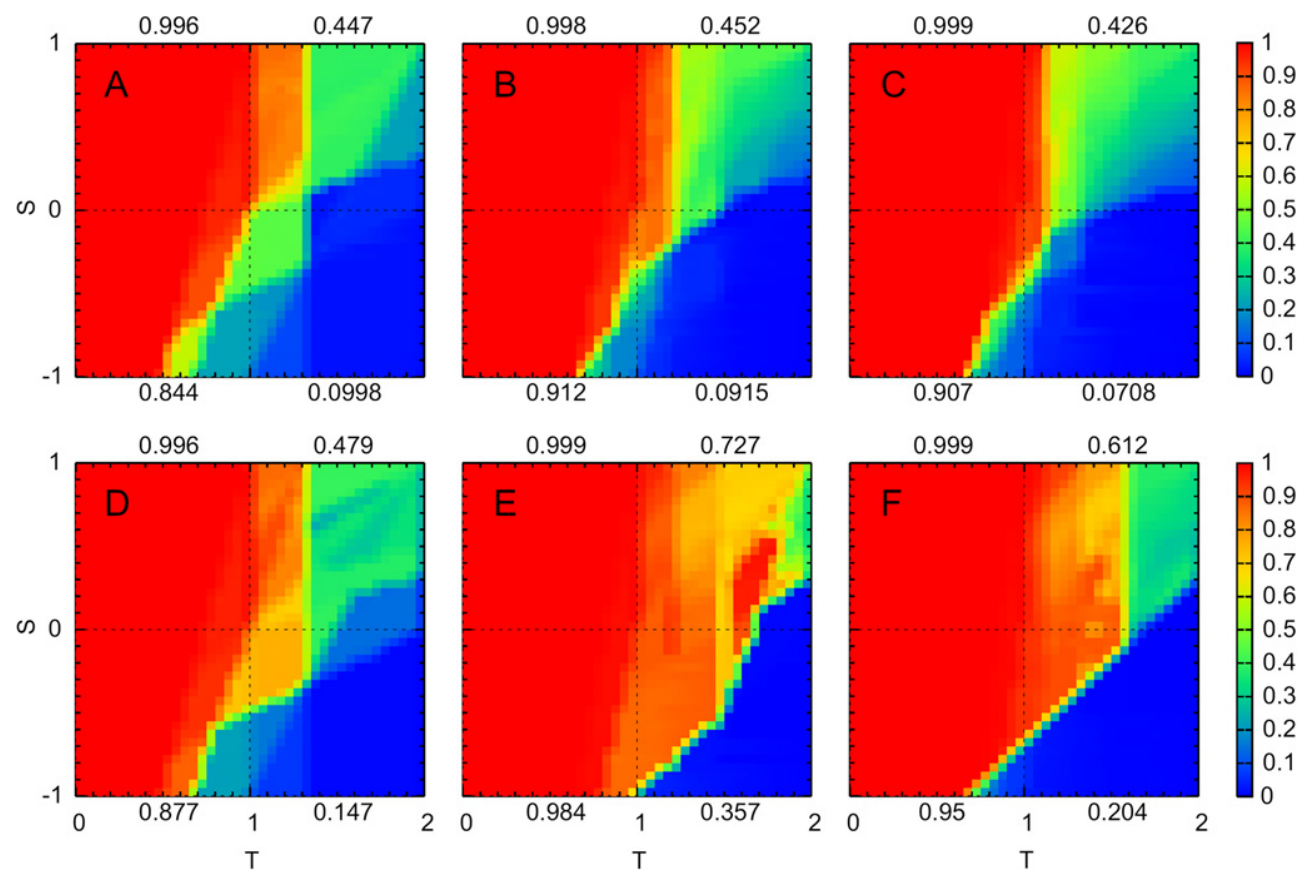

Fig. 15. Asymptotic density of cooperators $x^{*}$ in homogeneous random networks (upper row, A to C) compared to regular lattices (lower row, D to $\mathrm{F})$, with degrees $k=4(\mathrm{~A}, \mathrm{D}), 6(\mathrm{~B}, \mathrm{E})$ and $8(\mathrm{C}, \mathrm{F})$. The update rule is unconditional imitation and the initial density of cooperators is $x^{0}=0.5$. Again as in Fig. 13, spatial lattices have greater influence than random networks when the clustering coefficient is high $(k=6$ and 8$)$. In this case, however, the beneficial effect for cooperation goes well into Snowdrift and Prisoner's Dilemma quadrants.

cluster rule proposed by Hauert [74], which relates the outcome of the entire population to that of a cluster of this size. This property makes the evolutionary outcome quite independent of the initial density of cooperators, because even for a low initial density the probability that a suitable small cluster exists will be high for sufficiently large populations; see Fig. 17 D-F about the differences in initial conditions. Nevertheless, it is important to realize that this rule is based on the dynamics of flat interfaces and, therefore, it is only valid for unconditional imitation. Other update rules that also give rise to clusters, as replicator rule for example, develop interfaces with different shapes, rendering the particular case of flat interfaces irrelevant. As a consequence, the evolution outcome becomes dependent on the initial condition, as Fig. 17 A-C displays.

In summary, the relevant topological feature of these homogeneous networks, for the games and update rules considered so far, is the clustering of the network. Its effect depends largely on the update rule, and the most that can be said in general is that, besides not affecting Harmony games, it consistently promotes cooperation in Stag Hunt games.

\subsection{Synchronous vs asynchronous update}

Huberman and Glance [103] questioned the generality of the results reported by Nowak and May [73], in terms of the synchronicity of the update of strategies. Nowak and May used synchronous update, which means that every player is updated at the same time, so the population evolves in successive generations. Huberman and Glance, in contrast, employed asynchronous update (also called random sequential update), in which individuals are updated independently one by one, hence the neighborhood of each player always remains the same while her strategy is being updated. They showed that, for a particular game, the asymptotic cooperation obtained with synchronous update disappeared. This has become since then one of the most well-known and cited examples of the importance of synchronicity in the update of strategies in evolutionary models. Subsequent works have, in turn, criticized the importance of this issue, showing that the conclusions of [73] are robust [77,126], or restricting the effect reported by [103] to particular instances of Prisoner's Dilemma [78] or to the short memory of players [100]. Other works, however, in the 

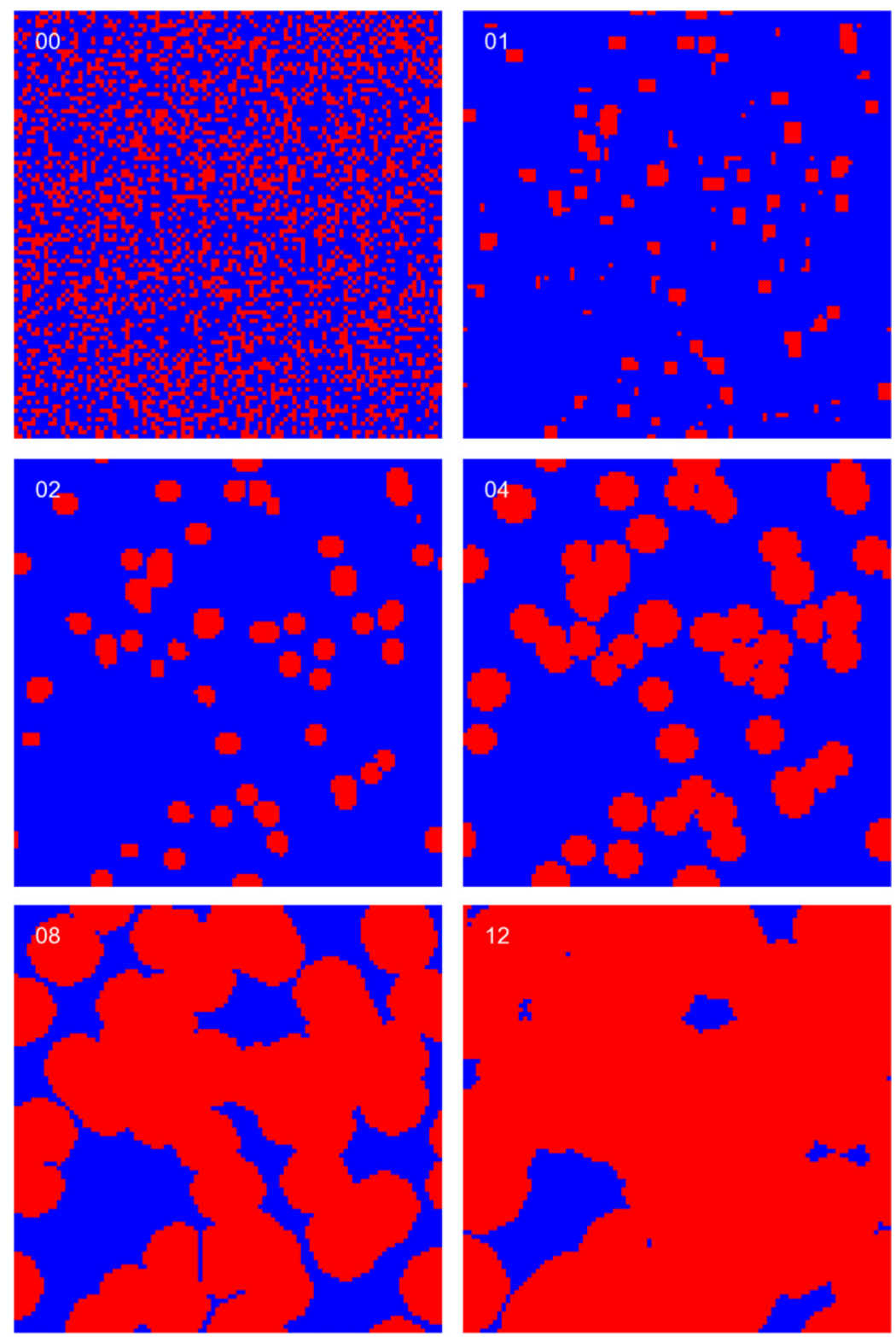

Fig. 16. Snapshots of the evolution of a population on a regular lattice of degree $k=8$, playing a Stag Hunt game $(S=-0.65$ and $T=0.65)$. Cooperators are displayed in red and defectors in blue. The update rule is unconditional imitation and the initial density of cooperators is $x^{0}=1 / 3$ (this lower value than that of Fig. 14 has been used to make the evolution longer and thus more easily observable). The upper left label shows the time step $t$. As with the replicator rule (see Fig. 14), during the initial time steps clusters emerge from cooperators with high local density of cooperators in their neighborhood. In this case, the interfaces advance deterministically at each time step, thus giving a special significance to flat interfaces and producing a much faster evolution than with the replicator rule (compare time labels with those of Fig. 14). (For interpretation of the references to color in this figure legend, the reader is referred to the web version of this article.)

different context of Snowdrift games $[95,96]$ have found that the influence on cooperation can be positive or negative, in the asynchronous case compared with the synchronous one.

We have thoroughly investigated this issue, finding that the effect of synchronicity in the update of strategies is the exception rather than the rule. With the replicator rule, for example, the evolutionary outcome in both cases is virtually identical, as Fig. 18 A-B shows. Moreover, in this case, the time evolution is also very similar (see Fig. 19 A-B). With unconditional imitation there are important differences only in one particular subregion of the space of 

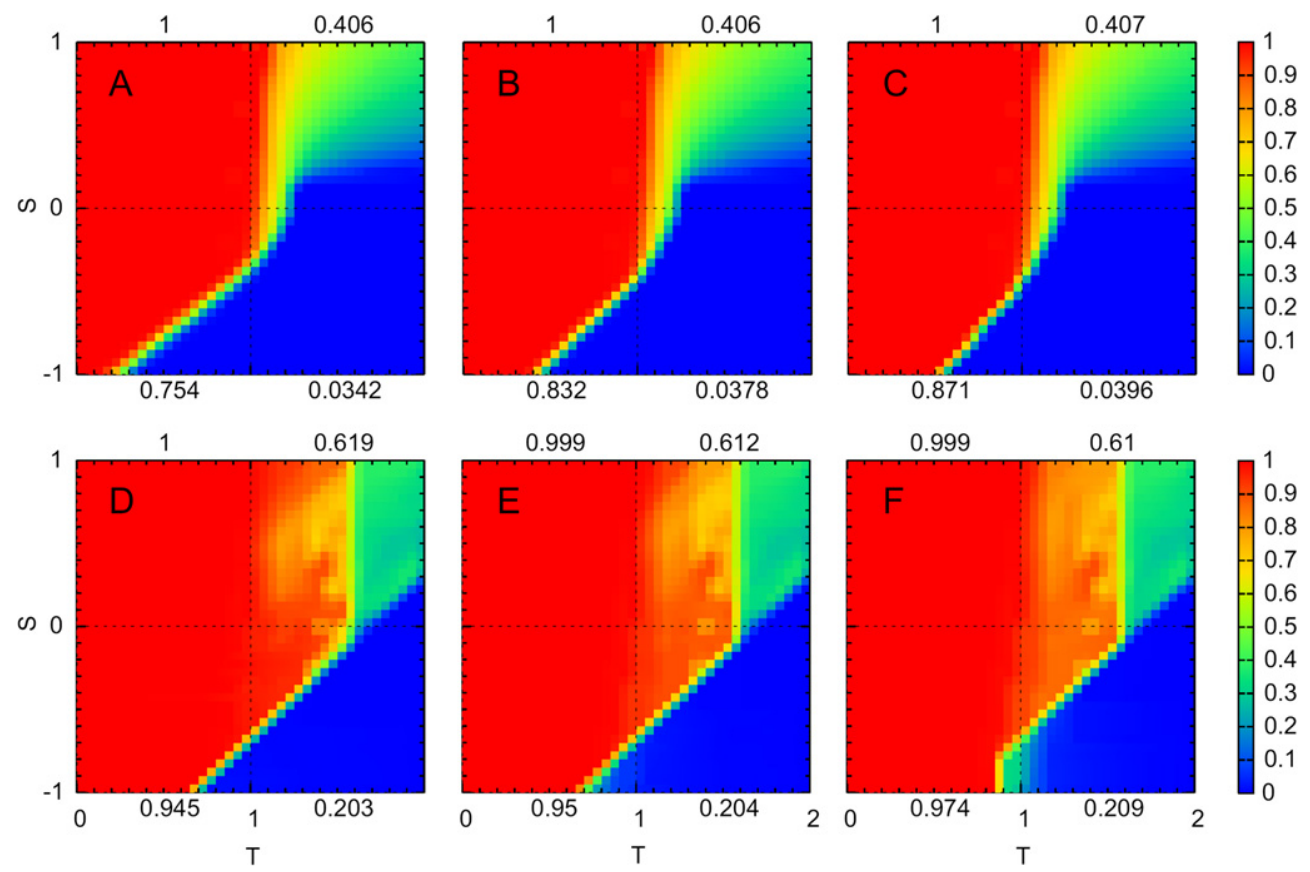

Fig. 17. Asymptotic density of cooperators $x^{*}$ in regular lattices of degree $k=8$, for different initial densities of cooperators $x^{0}=1 / 3(\mathrm{~A}, \mathrm{D})$, $1 / 2(\mathrm{~B}, \mathrm{E})$ and $2 / 3(\mathrm{C}, \mathrm{F})$. The update rules are the replicator rule (upper row, A to C) and unconditional imitation (lower row, D to F). With the replicator rule, the evolutionary outcome in Stag Hunt games depends on the initial condition, as is revealed by the displacement of the transition line between full cooperation and full defection. However, with unconditional imitation this transition line remains in the same position, thus showing the insensitivity to the initial condition. In this case, the outcome is determined by the presence of small clusters of cooperators in the initial random population, which takes place for a large range of values of the initial density of cooperators $x^{0}$.

parameters, corresponding mostly to Snowdrift games, to which the specific game studied by Huberman and Glance belongs (see Figs. $18 \mathrm{C}-\mathrm{D}$ and $19 \mathrm{C}-\mathrm{D}$ ).

\subsection{Heterogeneous networks}

The other important topological feature for evolutionary games was introduced by Santos and co-workers [75,127, 128], who studied the effect of degree heterogeneity, in particular scale-free networks. Their main result is shown in Fig. 20, which displays the variation in the evolutionary outcome induced by increasing the variance of the degree distribution in the population, from zero (homogeneous random networks) to a finite value (Erdős-Rényi random networks), and then to infinity (scale-free networks). The enhancement of cooperation as degree heterogeneity increases is very clear, specially in the region of Snowdrift games. The effect is not so strong, however, in Stag Hunt or Prisoner's Dilemma games. Similar conclusions are obtained with other scale-free topologies, as for example with Klemm-Eguíluz scale-free networks [129]. Very recently, it has been shown [130] that much as we discussed above for the case of spatial structures, clustering is also a factor improving the cooperative behavior in scale-free networks.

The positive influence on Snowdrift games is quite robust against changes in network degree and the use of other update rules. On the other hand, the influence on Stag Hunt and Prisoner's Dilemma games is quite restricted and very dependent on the update rule, as Fig. 21 reveals. In fact, with unconditional imitation cooperation is inhibited in Stag Hunt games as the network becomes more heterogeneous, whereas in Prisoner's Dilemmas it seems to have a maximum at networks with finite variance in the degree distribution.

A very interesting insight from the comparison between the effects of network clustering and degree heterogeneity is that they mostly affect games with one equilibrium in mixed strategies, and that in addition the effects on these games are different. This highlights the fact that they are different fundamental topological properties, which induce mechanisms of different nature. In the case of network clustering we have seen the formation and growth of clusters of cooperators. For network heterogeneity the phenomena is the bias and stabilization of the strategy oscillations in Snowdrift games towards the cooperative strategy [131,132], as we explain in the following. The asymptotic state of 


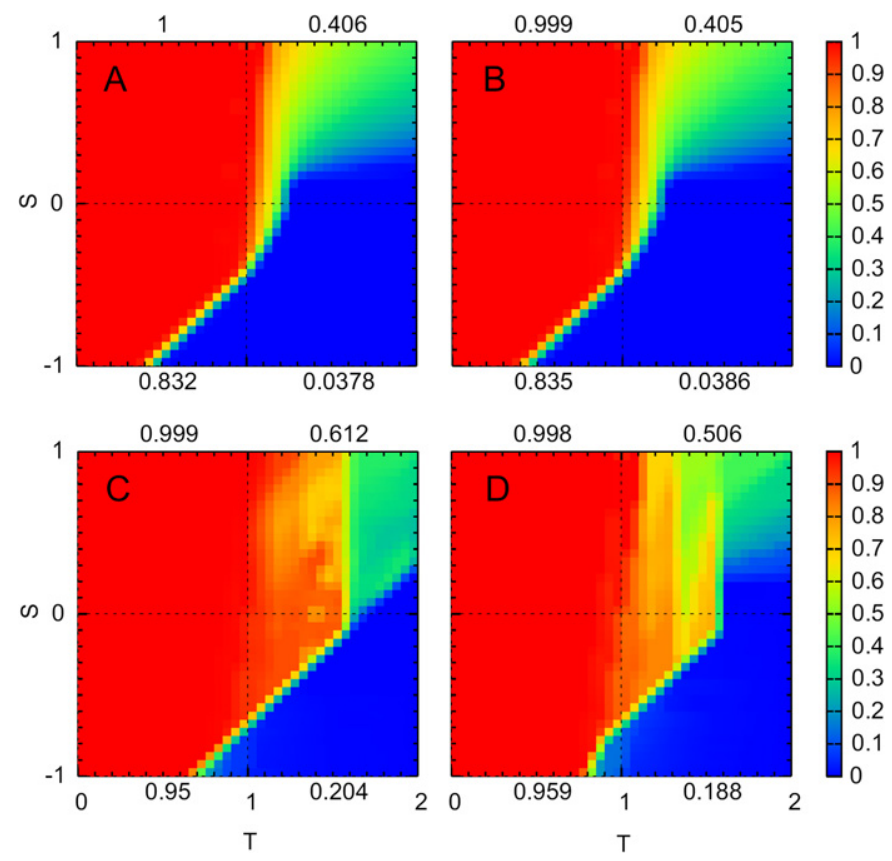

Fig. 18. Asymptotic density of cooperators $x^{*}$ in regular lattices of degree $k=8$, with synchronous update (left, A and C) compared to asynchronous (right, B and D). The update rules are the replicator rule (upper row) and unconditional imitation (lower row). The initial density of cooperators is $x^{0}=0.5$. For the replicator rule, the results are virtually identical, showing the lack of influence of the synchronicity of update on the evolutionary outcome. In the case of unconditional imitation the results are very similar, but there are differences for some points, specially Snowdrift games with $S \lesssim 0.3$ and $T>5 / 3 \approx 1.67$. The particular game studied by Huberman and Glance [103], which reported a suppression of cooperation due to asynchronous update, belongs to this region.

Snowdrift games in homogeneous networks consists of a mixed strategy population, where every individual oscillates permanently between cooperation and defection. Network heterogeneity tends to prevent this oscillation, making players in more connected sites more prone to be cooperators. At first, having more neighbors makes any individual receive more payoff, despite her strategy, and hence she has an evolutionary advantage. For a defector, this is a short-lived advantage, because it triggers the change of her neighbors to defectors, thus loosing payoff. A high payoff cooperator, on the contrary, will cause the conversion of her neighbors to cooperators, increasing even more her own payoff. These highly connected cooperators constitute the hubs that drive the population, fully or partially, to cooperation. It is clear that this mechanism takes place when cooperators collect more payoff from a greater neighborhood, independently of their neighbors' strategies. This only happens when $S>0$, which is the reason why the positive effect on cooperation of degree heterogeneity is mainly restricted to Snowdrift games.

\subsection{Best response update rule}

So far, we have dealt with imitative update rules, which are non-innovative. Here we present the results for an innovative rule, namely best response. With this rule each player chooses, with certain probability $p$, the strategy that is the best response for her current neighborhood. This rule is also referred to as myopic best response, because the player only takes into account the last evolution step to decide the optimum strategy for the next one. Compared to the rules presented previously, this one assumes more powerful cognitive abilities on the individual, as she is able to discern the payoffs she can obtain depending on her strategy and those of her neighbors, in order to chose the best response. From this point of view, it constitutes a next step in the sophistication of update rules.

An important result of the influence of this rule for evolutionary games was published in 2005 by Sysi-Aho and co-workers [94]. They studied the combined influence of this rule with regular lattices, in the same one-dimensional parameterization of Snowdrift games (41) that was employed by Hauert and Doebeli [93]. They reported a modification in the cooperator density at equilibrium, with an increase for some subrange of the parameter $T$ and a decrease for the other, as Fig. 22 shows. 

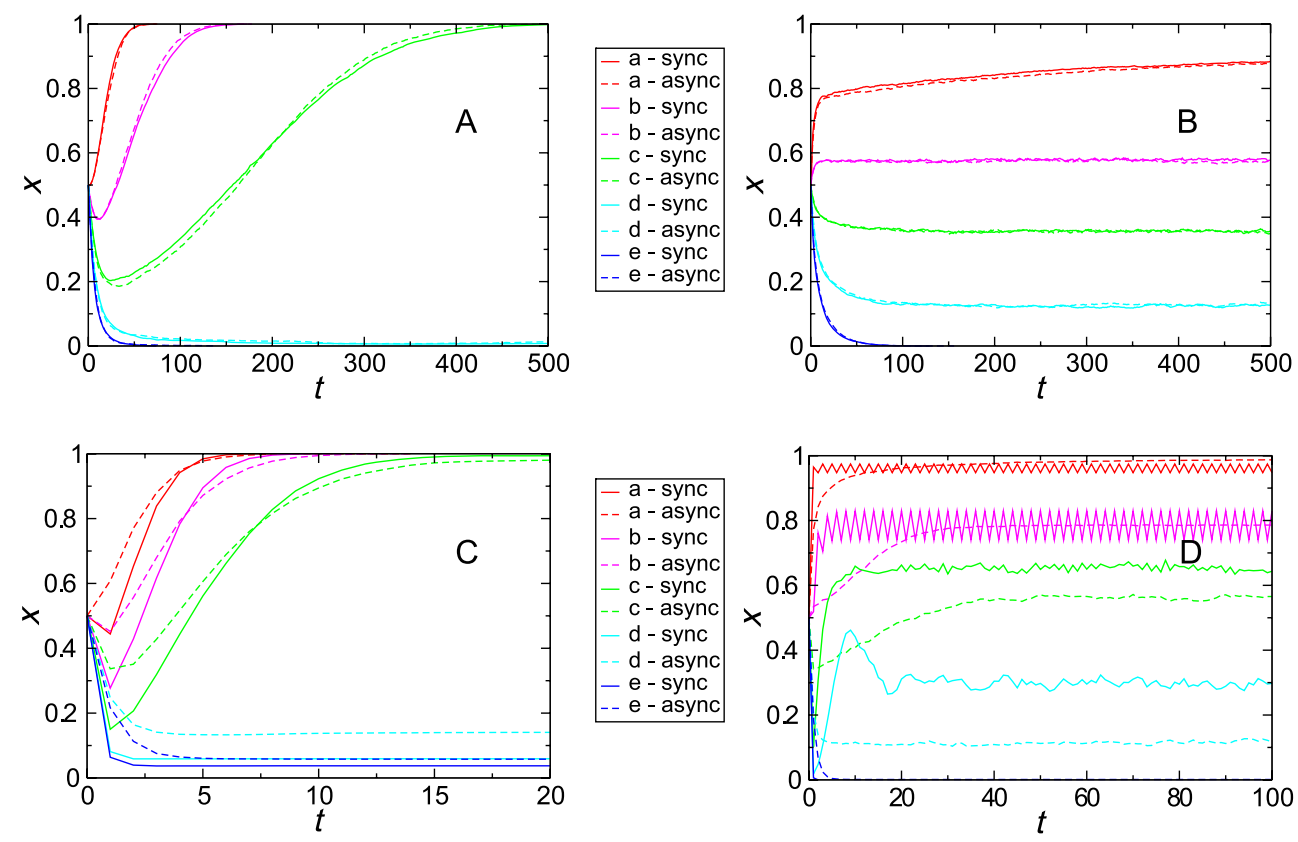

Fig. 19. Time evolution of the density of cooperators $x$ in regular lattices of degree $k=8$, for typical realizations of Stag Hunt (left, A and C) and Snowdrift games (right, B and D), with synchronous (continuous lines) or asynchronous (dashed lines) update. The update rules are the replicator rule (upper row) and unconditional imitation (lower row). The Stag Hunt games for the replicator rule (A) are: a, $S=-0.4, T=0.4 ; \mathrm{b}, S=-0.5$, $T=0.5 ; \mathrm{c}, S=-0.6, T=0.6 ; \mathrm{d}, S=-0.7, T=0.7 ; \mathrm{e}, S=-0.8, T=0.8$. For unconditional imitation the Stag Hunt games $(\mathrm{C})$ are: a, $S=-0.6$, $T=0.6 ; \mathrm{b}, S=-0.7, T=0.7 ; \mathrm{c}, S=-0.8, T=0.8$; d $S=-0.9, T=0.9 ; \mathrm{e}, S=-1.0, T=1.0$. The Snowdrift games are, for both update rules (B, D): a, $S=0.9, T=1.1$; b $S=0.7, T=1.3$; c, $S=0.5, T=1.5$; d, $S=0.3, T=1.7$; e, $S=0.1, T=1.9$. The initial density of cooperators is $x^{0}=0.5$. The time scale of the asynchronous realizations has been re-scaled by the size of the population, so $t$ hat for both kinds of update a time step represents the same number of update events in the population. Figures A and B show that, in the case of the replicator rule, not only the outcome but also the time evolution is independent of the update synchronicity. With unconditional imitation the results are also very similar for Stag Hunt (C), but somehow different in Snowdrift (D) for large $T$, displaying the influence of synchronicity in this subregion. Note that in all cases unconditional imitation yields a much faster evolution than the replicator rule.
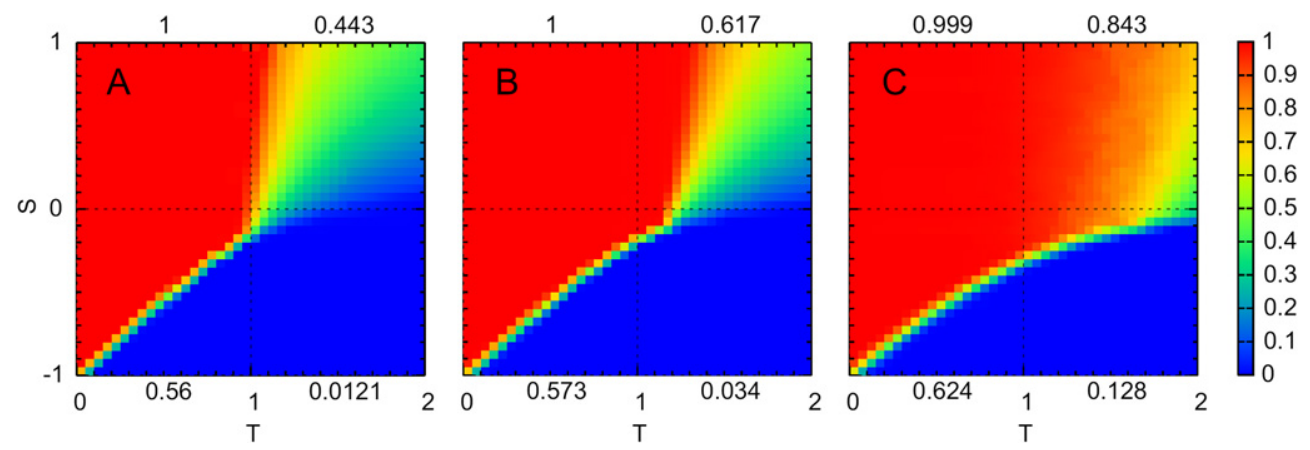

Fig. 20. Asymptotic density of cooperators $x^{*}$ with the replicator update rule, for model networks with different degree heterogeneity: homogeneous random networks (left, A), Erdős-Rényi random networks (middle, B) and Barabási-Albert scale-free networks (right, C). In all cases the average degree is $\bar{k}=8$ and the initial density of cooperators is $x^{0}=0.5$. As degree heterogeneity grows, from left to right, cooperation in Snowdrift games is clearly enhanced.

At the moment, it was intriguing that regular lattices had opposite effects (promotion or inhibition of cooperation) in some ranges of the parameter $T$, depending on the update rule used in the model. Very recently we have carried out a thorough investigation of the influence of this update rule on a wide range of networks [102], focusing on the key topological properties of network clustering and degree heterogeneity. The main conclusion of this study is that, with only one relevant exception, the best response rule suppresses the effect of population structure on evolutionary 

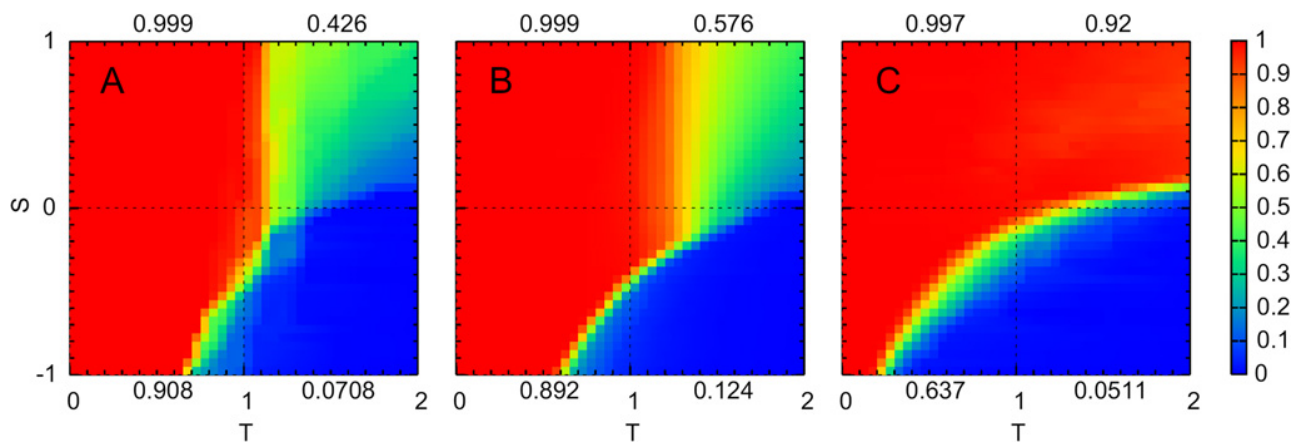

Fig. 21. Asymptotic density of cooperators $x^{*}$ with unconditional imitation as update rule, for model networks with different degree heterogeneity: homogeneous random networks (left, A), Erdős-Rényi random networks (middle, B) and Barabási-Albert scale-free networks (right, C). In all cases the average degree is $\bar{k}=8$ and the initial density of cooperators is $x^{0}=0.5$. As degree heterogeneity grows, from left to right, cooperation in Snowdrift games is enhanced again. In this case, however, cooperation is inhibited in Stag Hunt games and reaches a maximum in Prisoner's Dilemmas for Erdős-Rényi random networks.

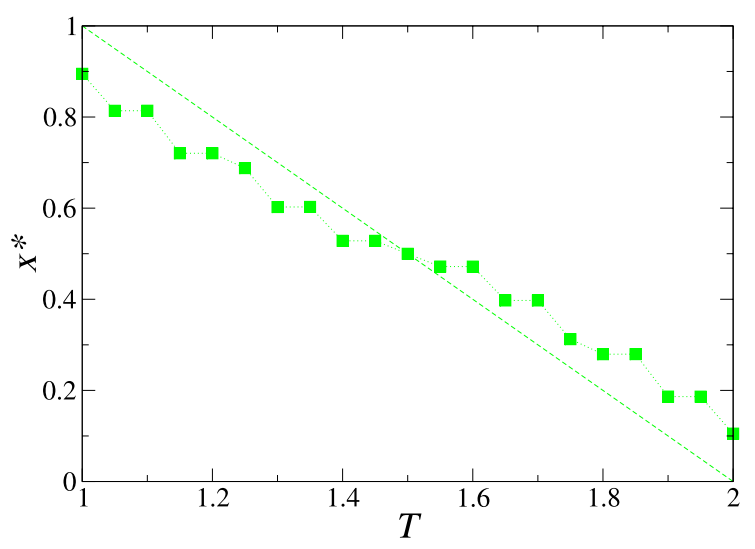

Fig. 22. Asymptotic density of cooperators $x^{*}$ in a square lattice with degree $k=8$ and best response as update rule, in the model with Snowdrift (41) studied by Sysi-Aho and co-workers [94]. The result for a well-mixed population is displayed as a reference. Note how the promotion or inhibition of cooperation does not follow the same variation as a function of $T$ than in the case with the replicator rule studied by Hauert and Doebeli [93] (Fig. 11).

games. Fig. 23 shows a summary of these results. In all cases the outcome is very similar to that of replicator dynamics on well-mixed populations (Fig. 9), despite the fact that the networks studied explore different options of network clustering and degree heterogeneity. The steps in the equilibrium density of Snowdrift games, as those reported in [94], show up in all cases, with slight variations which depend mostly on the mean degree of the network.

The exception to the absence of network influence is the case of regular lattices, and consists of a modification of the unstable equilibrium in Stag Hunt games, in the sense that it produces a promotion of cooperation for initial densities lower than 0.5 and a corresponding symmetric inhibition for greater densities. An example of this effect is given in Fig. 24, where the outcome should be compared to that of well-mixed populations in Fig. 9A. The reason for this effect is that the lattice creates special conditions for the advancement (or receding) of the interfaces of clusters of cooperators. We refer the interested reader to [102] for a detailed description of this phenomena. Very remarkably, in this case network clustering is not relevant, because the effect also takes place for degree $k=4$, at which there is no clustering in the network.

\subsection{Weak selection}

This far, we have considered the influence of population structure in the case of strong selection pressure, which means that the fitness of individuals is totally determined by the payoffs resulting from the game. In general this may not be the case, and then to relax this restriction the fitness can be expressed as $f=1-w+w \pi$ [133]. The parameter 

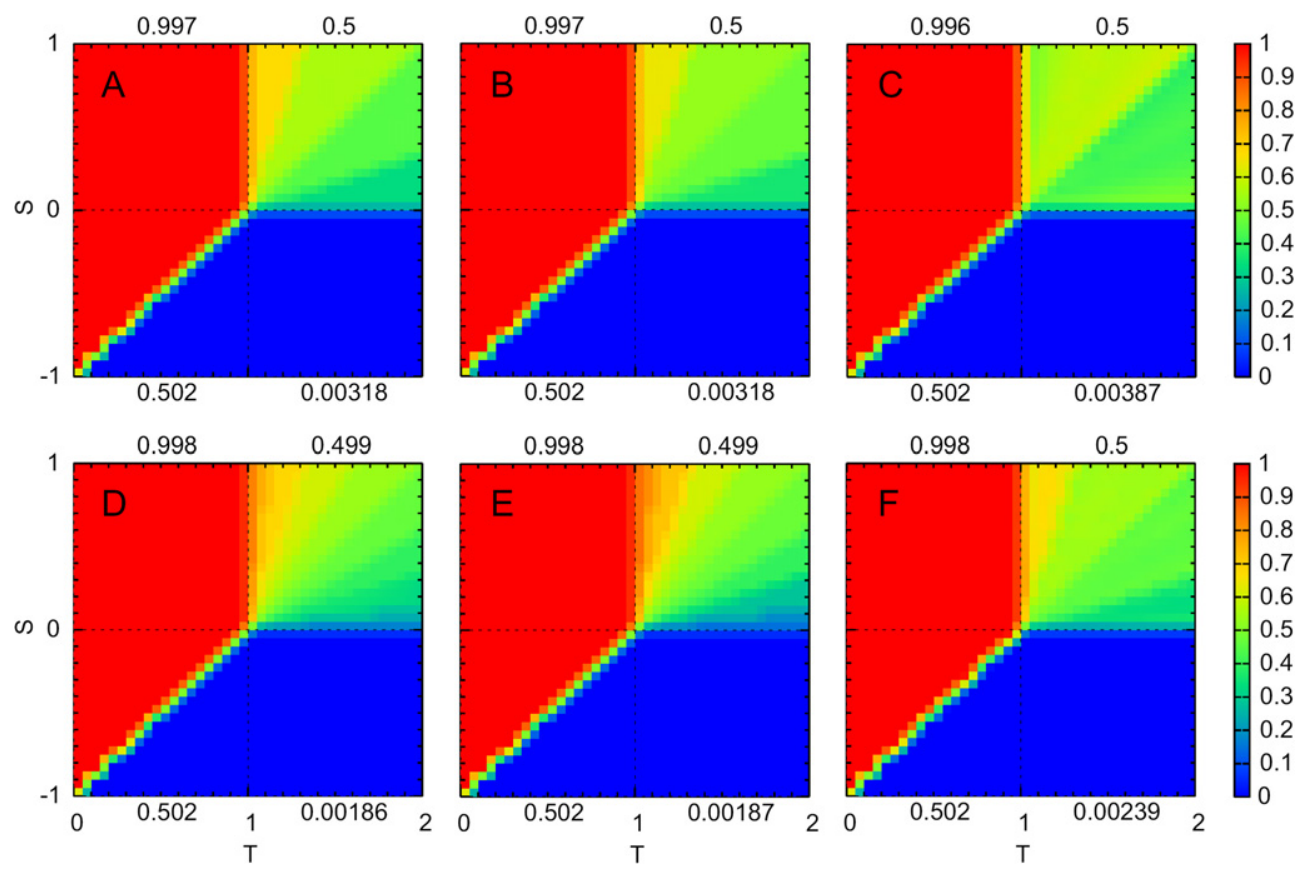

Fig. 23. Asymptotic density of cooperators $x^{*}$ in random (left, A and D), regular (middle, B and E), and scale-free networks (right, C and F) with average degrees $\bar{k}=4$ (upper row, A to C) and 8 (lower row, D to F). The update rule is best response with $p=0.1$ and the initial density of cooperators is $x^{0}=0.5$. Differences are negligible in all cases; note, however, that the steps appearing in the Snowdrift quadrant are slightly different.
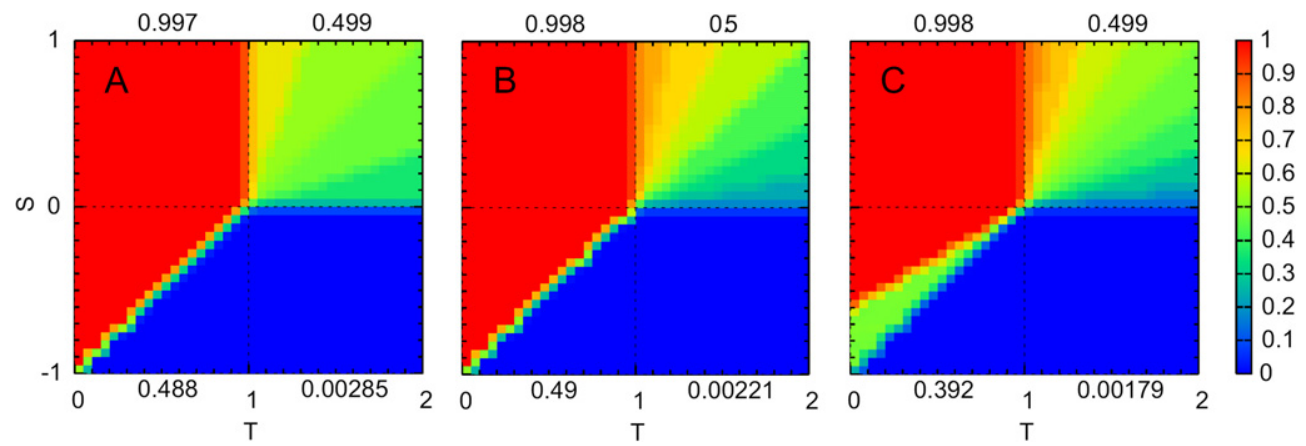

Fig. 24. Asymptotic density of cooperators $x^{*}$ in regular lattices with initial density of cooperators $x^{0}=1 / 3$. The degrees are $k=4$ (left, A), $k=6$ (middle, B) and $k=8$ (right, C). The update rule is best response with $p=0.1$. Comparing with Fig. 9A, there is a clear displacement of the boundary between full defection and full cooperation in Stag Hung games, which amounts to a promotion of cooperation. The widening of the border in panel $\mathrm{C}$ is a finite size effect, which disappears for larger populations. See main text for further details.

$w$ represents the intensity of selection and can vary between $w=1$ (strong selection limit) and $w \gtrsim 0$ (weak selection limit). With a different parameterization, this implements the same idea as the baseline fitness discussed in Section 3. We note that another interpretation has been recently proposed [134] for this limit, namely $\delta$-weak selection, which assumes that the game means much to the determination of reproductive success, but that selection is weak because mutant and wild-type strategies are very similar. This second interpretation leads to different results [134] and we do not deal with it here, but rather we stick with the first one, which is by far the most generally used.

The weak selection limit has the nice property of been tractable analytically. For instance, Ohtsuki and Nowak have studied evolutionary games on homogeneous random networks using this approach [135], finding an interesting relation with replicator dynamics on well-mixed populations. Using our normalization of the game (36), their main 

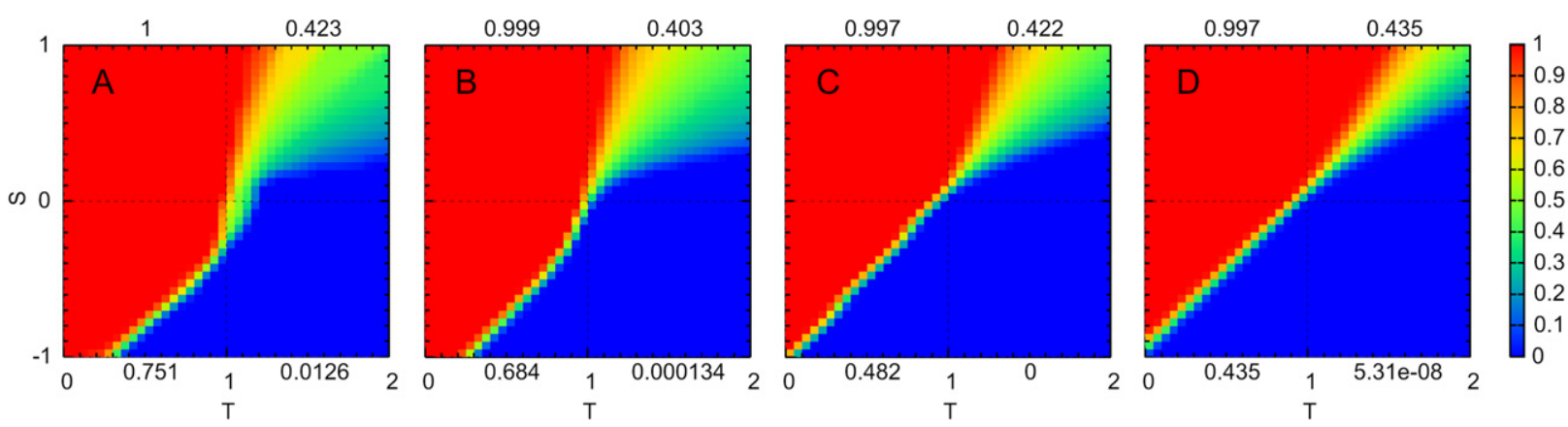

Fig. 25. Asymptotic density of cooperators $x^{*}$ in regular lattices of degree $k=8$, for the Fermi update rule with $\beta$ equal to 10 (A), 1 (B), 0.1 (C) and 0.01 (D). The initial density of cooperators is $x^{0}=0.5$. For high $\beta$ the result is quite similar to that obtained with the replicator rule (Fig. 13F). As $\beta$ decreases, or equivalently for weaker intensities of selection, the influence becomes smaller and more symmetrical between Stag Hunt and Snowdrift games.

result can be written as the following payoff matrix

$$
\left(\begin{array}{cc}
1 & S+\Delta \\
T-\Delta & 0
\end{array}\right)
$$

This means that the evolution in a population structured according to a random homogeneous network, in the weak selection limit, is the same as that of a well-mixed population with a game defined by this modified payoff matrix. The effect of the network thus reduces to the term $\Delta$, which depends on the game, the update rule and the degree $k$ of the network. With respect to the influence on cooperation it admits a very straightforward interpretation: If both the original and the modified payoff matrices correspond to a Harmony or Prisoner's Dilemma game, then there is logically no influence, because the population ends up equally in full cooperation or full defection; otherwise, cooperation is enhanced if $\Delta>0$, and inhibited if $\Delta<0$.

The actual values of $\Delta$, for the update rules Pairwise Comparison (PC), Imitation (IM) and Death-Birth (DB) (see [135] for full details), are

$$
\begin{aligned}
& \Delta_{\mathrm{PC}}=\frac{S-(T-1)}{k-2}, \\
& \Delta_{\mathrm{IM}}=\frac{k+S-(T-1)}{(k+1)(k-2)}, \\
& \Delta_{\mathrm{DB}}=\frac{k+3(S-(T-1))}{(k+3)(k-2)},
\end{aligned}
$$

$k$ being the degree of the network. A very remarkable feature of these expressions is that for every pair of games with parameters $\left(S_{1}, T_{1}\right)$ and $\left(S_{2}, T_{2}\right)$, if $S_{1}-T_{1}=S_{2}-T_{2}$ then $\Delta_{1}=\Delta_{2}$. Hence the influence on cooperation for such a pair of games, even if one is a Stag Hunt and the other is a Snowdrift, will be the same. This stands in stark contrast to all the reported results with strong selection, which generally exhibit different, and in many cases opposite, effects on both games. Besides this, as the term $S-(T-1)$ is negative in all Prisoner's Dilemmas and half the cases of Stag Hunt and Snowdrift games, the beneficial influence on cooperation is quite reduced for degrees $k$ as those considered above [101].

Another way to investigate the influence of the intensity of selection if to employ the Fermi update rule, presented above, which allows to study numerically the effect of varying the intensity of selection on any network model. Figs. 25 and 26 display the results obtained, for different intensities of selection, on networks that are prototypical examples of strong influence on evolutionary games, namely regular lattices with high clustering and scale-free networks, with large degree heterogeneity. In both cases, as the intensity of selection is reduced, the effect of the network becomes weaker and more symmetrical between Stag Hunt and Snowdrift games. Therefore, these results show that the strong and weak selection limits are not comparable from the viewpoint of the evolutionary outcome, and that weak selection largely inhibits the influence of population structure. 

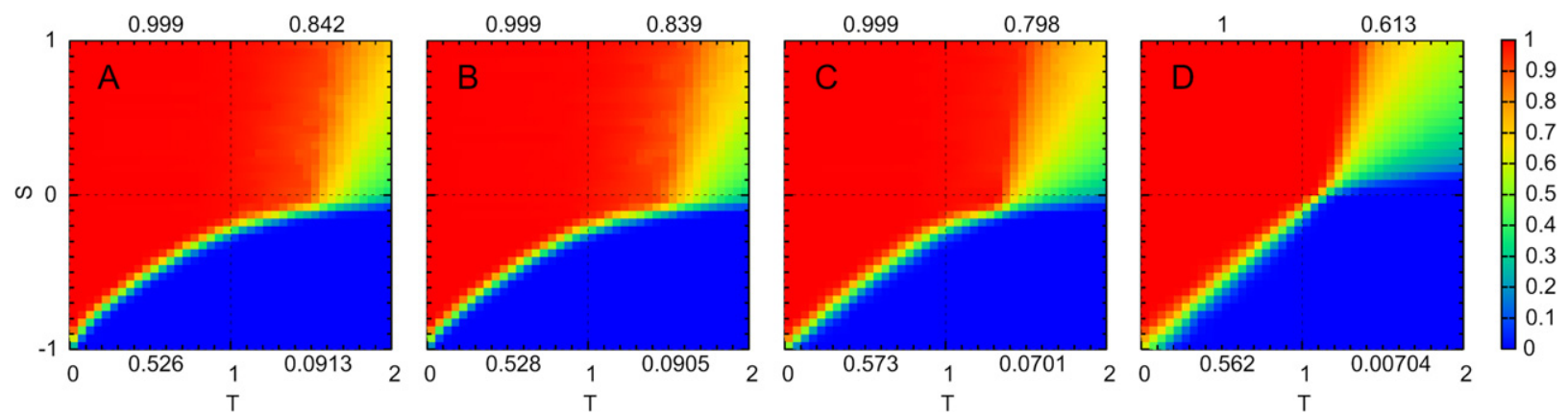

Fig. 26. Asymptotic density of cooperators $x^{*}$ in Barabási-Albert scale-free networks of average degree $\bar{k}=8$, for the Fermi update rule with $\beta$ equal to 10 (A), 1 (B), 0.1 (C) and 0.01 (D). The initial density of cooperators is $x^{0}=0.5$. As in Fig. 25 , for high $\beta$ the result is quite similar to that obtained with the replicator rule (Fig. 20C), and analogously, as $\beta$ decreases the influence of the network becomes smaller and more symmetrical between Stag Hunt and Snowdrift games.

\section{Conclusion and future prospects}

In this review, we have discussed non-mean-field effects on evolutionary game dynamics. Our reference framework for comparison has been the replicator equation, a pillar of modern evolutionary game theory that has produced many interesting and fruitful insights on different fields. Our purpose here has been to show that, in spite of its many successes, the replicator equation is only a part of the story, much in the same manner as mean-field theories have been very important in physics but they cannot (nor are they intended to) describe all possible phenomena. The main issues we have discussed are the influence of fluctuations, by considering the existence of more than one time scale, and of spatial correlations, through the constraints on interaction arising from an underlying network structure. In doing so, we have shown a wealth of evidence supporting our first general conclusion: Deviations with respect to the hypothesis of a well-mixed population (including nonlinear dependencies of the fitness on the payoff or not) have a large influence on the outcome of the evolutionary process and in a majority of cases do change the equilibria structure, stability and/or basins of attraction.

The specific question of the existence of different time scales was discussed in Section 3. This is a problem that has received some attention in economics but otherwise it has been largely ignored in biological contexts. In spite of this, we have shown that considering fast evolution in the case of Ultimatum game may lead to a non-trivial, unexpected conclusion: That individual selection may be enough to explain the experimental evidence that people do not behave rationally. This is an important point in so far as, to date, simple individual selection was believed not to provide an understanding of the phenomena of altruistic punishment reported in many experiments [56]. We thus see that the effect of different time scales might be determinant and therefore must be considered among the relevant factors with an influence on evolutionary phenomena.

This conclusion is reinforced by our general study of symmetric $2 \times 2$ symmetric games, that shows that the equilibria of about half of the possible games change when considering fast evolution. Changes are particularly surprising in the case of the Harmony game, in which it turns out that when evolution is fast, the selected strategy is the "wrong" one, meaning that it is the less profitable for the individual and for the population. Such a result implies that one has to be careful when speaking of adaptation through natural selection, because in this example we have a situation in which selection leads to a bad outcome through the influence of fluctuations. It is clear that similar instances may arise in many other problems. On the other hand, as for the particular question of the emergence of cooperation, our results imply that in the framework of the classical $2 \times 2$ social dilemmas, fast evolution is generally bad for the appearance of cooperative behavior.

The results reported here concerning the effect of time scales on evolution are only the first ones in this direction and, clearly, much remains to be done. In this respect, we believe that it would be important to work out the case of asymmetric $2 \times 2$ games, trying to reveal possible general conclusions that apply to families of them. The work on the Ultimatum game [64] is just a first example, but no systematic analysis of asymmetric games has been carried out. A subsequent extension to games with more strategies would also be desirable; indeed, the richness of the structures arising in those games (such as, e.g., the rock-scissors-papers game [11]) suggests that considering fast evolution may lead to quite unexpected results. This has been very recently considered in the framework of the evolutionary minority 
game [136] (where many strategies are possible, not just two or three) once again from an economics perspective [137]; the conclusion of this paper, namely that there is a phase transition as a function of the time scale parameter that can be observed in the predictability of market behavior is a further hint of the interest of this problem.

In Section 4 we have presented a global view of the influence of population structure on evolutionary games. We have seen a rich variety of results, of unquestionable interest, but that on the downside reflect the non-generality of this kind of evolutionary models. Almost every detail in the model matters on the outcome, and some of them dramatically.

We have provided evidence that population structure does not necessarily promote cooperation in evolutionary game theory, showing instances in which population structure enhances or inhibits it. Nonetheless, we have identified two topological properties, network clustering and degree heterogeneity, as those that allow a more unified approach to the characterization and understanding of the influence of population structure on evolutionary games. For certain subset of update rules, and for some subregion in the space of games, they induce consistent modifications in the outcome. In summary, network clustering has a positive impact on cooperation in Stag Hunt games and degree heterogeneity in Snowdrift games. Therefore, it would be reasonable to expect similar effects in other networks which share these key topological properties. In fact, there is another topological feature of networks that conditions evolutionary games, albeit of a different type: The community structure [108,109]. Communities are subgraphs of densely interconnected nodes, and they represent some kind of mesoscopic organization. A recent study [76] has pointed out that communities may have their own effects on the game asymptotics in otherwise similar graphs, but more work is needed to assess this influence.

On the other hand, the importance of the update rules cannot be overstated. We have seen that for the best response and Fermi rules even these "robust" effects of population structure are greatly reduced. It is very remarkable from a application point of view that the influence of population structure is inhibited so greatly when update rules more sophisticated than merely imitative ones are considered, or when the selection pressure is reduced. It is evident that a sound justification of several aspects of the models is mandatory for applications. Crucial details, as the payoff structure of the game, the characteristics of the update rule or the main topological features of the network are critical for obtaining significant results. For the same reasons, unchecked generalizations of the conclusions obtained from a particular model, which go beyond the kind of game, the basic topology of the network or the characteristics of the updated rule, are very risky in this field of research. Very easily the evolutionary outcome of the model could change dramatically, making such generalizations invalid.

This conclusion has led a number of researchers to address the issue from a further evolutionary viewpoint: Perhaps, among the plethora of possible networks one can think of, only some of them (or some values of their magnitudes) are really important, because the rest are not found in actual situations. This means that networks themselves may be subject to natural selection, i.e., they may co-evolve along with the game under consideration. This promising idea has already been proposed [138-145] and a number of interesting results, which would deserve a separate review on their own right, ${ }^{7}$ have been obtained regarding the emergence of cooperation. In this respect, it has been observed that co-evolution seems to favor the stabilization of cooperative behavior, more so if the network is not rewired from a preexisting one but rather grows upon arrival of new players [147]. A related approach, in which the dynamics of the interaction network results from the mobility of players over a spatial substrate, has been the focus of recent works $[148,149]$. Albeit these lines of research are appealing and natural when one thinks of possible applications, we believe the same caveat applies: It is still too early to draw general conclusions and it might be that details would be again important. Nevertheless, work along these lines is needed to assess the potential applicability of these types of models. Interestingly, the same approach is also being introduced to understand which strategy update rules should be used, once again as a manner to discriminate among the very many possibilities. This was pioneered by Harley [150] (see also the book by Maynard Smith [6], where the paper by Harley is presented as a chapter) and a few works have appeared in the last few years [151-155]; although the available results are too specific to allow for a glimpse of any general feature, they suggest that continuing this research may render fruitful results.

We thus reach our main conclusion: The outcome of evolutionary game theory depends to a large extent on the details, a result that has very important implications for the use of evolutionary game theory to model actual biological, sociological or economical systems. Indeed, in view of this lack of generality, one has to look carefully at the main factors involved in the situation to be modeled because they need to be included as close as necessary to reality to

$\overline{7}$ For a first attempt, see Section 5 of [146]. 
produce conclusions relevant for the case of interest. Note that this does not mean that it is not possible to study evolutionary games from a more general viewpoint; as we have seen above, general conclusions can be drawn, e.g., about the beneficial effects of clustering for cooperation or the key role of hubs in highly heterogeneous networks. However, what we do mean is that one should not take such a general conclusions for granted when thinking of a specific problem or phenomenon, because it might well be that some of its specifics render these abstract ideas unapplicable. On the other hand, it might be possible that we are not looking at the problem in the right manner; there may be other magnitudes we have not identified yet that allow for a classification of the different games and settings into something similar to universality classes. Whichever the case, it seems clear to us that much research is yet to be done along the lines presented here. We hope that this review encourages others to walk off the beaten path in order to make substantial contributions to the field.

\section{Acknowledgements}

This work has been supported by projects MOSAICO, from the Spanish Ministerio de Educación y Ciencia, and MOSSNOHO and SIMUMAT, from the Comunidad Autónoma de Madrid.

\section{Appendix A. Characterization of birth-death processes}

One of the relevant quantities to determine in a birth-death process is the probability $c_{n}$ that, starting from state $n$, the process ends eventually absorbed into the absorbing state $n=N$. There is a simple relationship between $c_{n}$ and the stochastic matrix $P$, namely

$$
c_{n}=P_{n, n-1} c_{n-1}+P_{n, n} c_{n}+P_{n, n+1} c_{n+1}, \quad 0<n<N,
$$

with the obvious boundary conditions $c_{0}=0$ and $c_{N}=1$. The solution to this equation is [69]

$$
c_{n}=\frac{Q_{n}}{Q_{N}}, \quad Q_{n}=\sum_{j=0}^{n-1} q_{j}, \quad q_{0}=1, \quad q_{j}=\prod_{i=1}^{j} \frac{P_{i, i-1}}{P_{i, i+1}} \quad(j>0) .
$$

Another relevant quantity is $v_{k, n}$, the expected number of visits that, starting from state $k$, the process pays to site $n$ before it enters one absorbing state. If $V=\left(v_{k, n}\right)$, with $0<k, n<N$, then

$$
V=I+R+R^{2}+\cdots=(I-R)^{-1},
$$

where $I$ is the identity matrix and $R$ is the submatrix of $P$ corresponding to the transient (non-absorbing) states. The series converges because $R$ is substochastic [70]. Thus $V$ fulfills the equation $V=V R+I$, which amounts to an equation similar to (46) for every row of $V$, namely

$$
v_{k, n}=v_{k, n-1} P_{n-1, n}+v_{k, n} P_{n, n}+v_{k, n+1} P_{n+1, n}+\delta_{k, n}, \quad 0<k, n<N,
$$

where $\delta_{k, n}=1$ if $k=n$ and 0 otherwise. Contrary to what happens with Eq. (46), this equation has no simple solution and it is better solved as in (48). Finally, $\tau_{k}$, the number of steps before absorption occurs into any absorbing state, when starting at state $k$, is obtained as

$$
\tau_{k}=\sum_{n=1}^{N-1} v_{k, n}
$$

\section{Appendix B. Absorption probability in the hypergeometric case}

For the special case in which

$$
\frac{P_{n, n-1}}{P_{n, n+1}}=\frac{\alpha n+\beta}{\alpha(n+1)+\gamma}
$$


the absorption probability into state $n=N, c_{n}$, can be obtained in closed form. According to (47) the sequence $q_{j}$ fulfills the hypergeometric relation

$$
\frac{q_{j}}{q_{j-1}}=\frac{\alpha j+\beta}{\alpha(j+1)+\gamma},
$$

from which

$$
(\alpha(j+1)+\gamma) q_{j}=(\alpha j+\beta) q_{j-1} .
$$

Adding this equation up for $j=1, \ldots, n-1$ we get

$$
\alpha \sum_{j=1}^{n-1}(j+1) q_{j}+\gamma\left(Q_{n}-1\right)=\alpha \sum_{j=0}^{n-2}(j+1) q_{j}+\beta\left(Q_{n}-q_{n-1}\right),
$$

and therefore

$$
(\gamma-\beta) Q_{n}=\gamma+\alpha-(\beta+\alpha n) q_{n-1} .
$$

Thus, provided $\gamma \neq \beta$, we obtain

$$
Q_{n}=\frac{\gamma+\alpha}{\gamma-\beta}\left[1-\prod_{j=1}^{n} \frac{\alpha j+\beta}{\alpha j+\gamma}\right] \text {. }
$$

If $\alpha=0$ this has the simple form

$$
Q_{n}=\frac{\gamma}{\gamma-\beta}\left[1-(\beta / \gamma)^{n}\right] \text {. }
$$

If $\alpha \neq 0$, then we can rewrite

$$
Q_{n}=\frac{\gamma+\alpha}{\gamma-\beta}\left[1-\frac{\Gamma(\beta / \alpha+n+1) \Gamma(\gamma / \alpha+1)}{\Gamma(\gamma / \alpha+n+1) \Gamma(\beta / \alpha+1)}\right] .
$$

The case $\gamma=\beta$ can be obtained from (55) or as the limit of expression (58) when $\gamma \rightarrow \beta$. Both ways yield

$$
Q_{n}=\sum_{j=1}^{n} \frac{\alpha+\gamma}{\alpha j+\gamma} \text {. }
$$

\section{References}

[1] Darwin C. On the origin of species by means of natural selection, or the preservation of favoured races in the struggle for life. 1st edition. London: John Murray; 1859.

[2] Roughgarden J. Theory of population genetics and evolutionary ecology: An introduction. New York: MacMillan Publishing Co.; 1979.

[3] Peliti L. Fitness landscapes and evolution. In: Riste T, Sherrington D, editors. Physics of biomaterials: Fluctuations, self-assembly and evolution. Dordrecht: Kluwer Academic; 1996. p. 287-308.

[4] Drossel B. Biological evolution and statistical physics. Adv Phys 2001;50:209-95.

[5] Ewens WJ. Mathematical population genetics. 2nd edition. New York: Springer; 2004.

[6] Maynard Smith J. Evolution and the theory of games. Cambridge: Cambridge University Press; 1982.

[7] Gould SJ, Vrba ES. Exaptation - a missing term in the science of form. Paleobiology 1982;8:4-15.

[8] von Neumann J, Morgenstern O. The theory of games and economic behavior. Princeton: Princeton University Press; 1947.

[9] Gintis H. Game theory evolving. Princeton: Princeton University Press; 2000.

[10] Nowak MA. Evolutionary dynamics: Exploring the equations of life. Cambridge: The Belknap Press of Harvard University Press; 2006.

[11] Hofbauer J, Sigmund K. Evolutionary games and population dynamics. Cambridge: Cambridge University Press; 1998.

[12] Taylor PD, Jonker L. Evolutionarily stable strategies and game dynamics. Math Biosci 1978;40:145-56.

[13] Nash J. Equilibrium points in $n$-person games. Proc Natl Acad Sci USA 1950;36:48-9.

[14] Pennisi E. How did cooperative behavior evolve? Science 2005;309:93.

[15] Hamilton WD. The genetical evolution of social behaviour I. J Theor Biol 1964;7:1-16.

[16] Hamilton WD. The genetical evolution of social behaviour II. J Theor Biol 1964;7:17-52.

[17] Trivers RL. The evolution of reciprocal altruism. Q Rev Biol 1971;46:35-57.

[18] Hammerstein P, editor. Genetic and cultural evolution of cooperation (Dahlem Workshop Report 90). Cambridge: MIT Press; 2003. 
[19] Nowak MA. Five rules for the evolution of cooperation. Science 2006;314:1560-3.

[20] Szabó G, Fáth G. Evolutionary games on graphs. Phys Rep 2007;446:97-216.

[21] Hofbauer J, Sigmund K. Evolutionary game dynamics. Bull Am Math Soc 2003;40:479-519.

[22] Rapoport A, Chammah AM. Prisoner's dilemma. Ann Arbor: University of Michigan Press; 1965.

[23] Sugden R. The economics of rights, co-operation and welfare. Oxford: Basil Blackwell; 1986.

[24] Maynard Smith J, Price G. The logic of animal conflict. Nature 1973;246:15-8.

[25] Vega-Redondo F. Economics and the theory of games. Cambridge: Cambridge University Press; 2003.

[26] Schuster P, Sigmund K. Replicator dynamics. J Theor Biol 1983;100:533-8.

[27] Traulsen A, Claussen JC, Hauert C. Coevolutionary dynamics: From finite to infinite populations. Phys Rev Lett 2005;95:238701.

[28] Claussen JC, Traulsen A. Non-Gaussian fluctuations arising from finite populations: Exact results for the evolutionary moran process. Phys Rev E 2005;71:25101.

[29] Traulsen A, Claussen JC, Hauert C. Coevolutionary dynamics in large, but finite populations. Phys Rev E 2006;74:011901.

[30] Claussen JC. Discrete stochastic processes, replicator and Fokker-Planck equations of coevolutionary dynamics in finite and infinite populations. Banach Center Publications 2008;80:17-31.

[31] Fogel DB, Fogel GB, Andrews PC. On the instability of evolutionary stable strategies. Biosystems 1997;44:135-52.

[32] Fogel GB, Andrews PC, Fogel DB. On the instability of evolutionary stable strategies in small populations. Ecol Modelling 1998;109:283-94.

[33] Page KM, Nowak MA. Unifying evolutionary dynamics. J Theor Biol 2002;219:93-8.

[34] Price GR. Selection and covariance? Nature 1970;227:520-1.

[35] Frank SA. George Price's contributions to evolutionary genetics. J Theor Biol 1995;175:373-88.

[36] Kollock P. Social dilemmas: The anatomy of cooperation. Annu Rev Sociol 1998;24:183-214.

[37] Maynard Smith J, Szathmáry E. The major transitions in evolution. Oxford: Oxford University Press; 1995.

[38] Hendry AP, Kinnison MT. Perspective: The pace of modern life: Measuring rates of contemporary microevolution. Evolution 1999;53:163753.

[39] Hendry AP, Wenburg JK, Bentzen P, Volk EC, Quinn TP. Rapid evolution of reproductive isolation in the wild: Evidence from introduced salmon. Science 2000;290:516-8.

[40] Yoshida T, Jones LE, Ellner SP, Fussmann GF, Hairston Jr. NG. Rapid evolution drives ecological dynamics in a predator-prey system. Nature 2003;424:303-6.

[41] Fagen RM. When doves conspire: Evolution of non-damaging fighting tactics in a non-random encounter animal conflict model. Am Nat 1980;115:858-69.

[42] Skyrms B. The stag hunt and evolution of social structure. Cambridge: Cambridge University Press; 2003.

[43] Harsanyi JC, Selten R. A general theory of equilibrium selection in games. Cambridge, MA: MIT; 1988.

[44] Macy MW, Flache A. Learning dynamics in social dilemmas. Proc Natl Acad Sci USA 2002;99:7229-36.

[45] Kandori M, Mailath GJ, Roy R. Learning, mutation and long-run equilibria in games. Econometrica 1993;61:29-56.

[46] Foster D, Young PH. Stochastic evolutionary game dynamics. Theor Popul Biol 1990;38:219-32.

[47] Robson AJ, Vega-Redondo F. Efficient equilibrium selection in evolutionary games with random matching. J Econ Theory 1996;70:65-92.

[48] Miekisz J. Equilibrium selection in evolutionary games with random matching of players. J Theor Biol 2005;232:47-53.

[49] Wölfing B, Traulsen A. Stochastic sampling of interaction partners versus deterministic payoff assignment. J Theor Biol 2009;257:689-95.

[50] Boylan RT. Laws of large numbers for dynamical systems with randomly matched individuals. J Econ Theory 1992;57:473-504.

[51] Boylan RT. Continuous approximation of dynamical systems with randomly matched individuals. J Econ Theory 1995;66:615-25.

[52] Licht AN. Games commissions play: $2 \times 2$ games of international securities regulation. Yale J Int Law 1999;24:61-125.

[53] Güth W, Schmittberger R, Schwarze B. An experimental analysis of ultimate bargaining. J Econ Behav Org 1982;3:367-88.

[54] Henrich J, Boyd R, Bowles S, Camerer C, Fehr E, Gintis H, editors. Foundations of human sociality: Economic experiments and ethnographic evidence from fifteen small-scale societies. Oxford: Oxford University Press; 2004.

[55] Nowak MA, Sigmund K. Evolution of indirect reciprocity by image scoring. Nature 1998;393:573-7.

[56] Camerer CF. Behavioral game theory. Princeton: Princeton University Press; 2003.

[57] Fehr E, Fischbacher U. The nature of human altruism. Nature 2003;425:785-91.

[58] Axelrod R, Hamilton WD. The evolution of cooperation. Science 1981;211:1390-6.

[59] Axelrod R. The evolution of cooperation. New York: Basic Books; 1984.

[60] Gintis H. Strong reciprocity and human sociality. J Theor Biol 2000;206:169-79.

[61] Fehr E, Fischbacher U, Gächter S. Strong reciprocity, human cooperation and the enforcement of social norms. Hum Nat 2002;13:1-25.

[62] Page K, Nowak M. A generalized adaptive dynamics framework can describe the evolutionary Ultimatum game. J Theor Biol 2000;209:1739.

[63] Page K, Nowak M. Empathy leads to fairness. Bull Math Biol 2002;64:1101-16.

[64] Sánchez A, Cuesta JA. Altruism may arise from individual selection. J Theor Biol 2005;235:233-40.

[65] Roca CP, Cuesta JA, Sánchez A. Time scales in evolutionary dynamics. Phys Rev Lett 2006;97:158701.

[66] Roca CP, Cuesta JA, Sánchez A. The importance of selection rate in the evolution of cooperation. Eur Phys J Special Topics 2007;143:51-8.

[67] Moran PAP. The statistical processes of evolutionary theory. Oxford: Clarendon Press; 1962.

[68] Taylor C, Fudenberg D, Sasaki A, Nowak MA. Evolutionary game dynamics in finite populations. Bull Math Biol 2004;66:1621-44.

[69] Karlin S, Taylor HM. A first course in stochastic processes. 2nd edition. New York: Academic Press; 1975.

[70] Grinstead CM, Snell JL. Introduction to probability. 2nd edition. Providence: American Mathematical Society; 1997.

[71] Rapoport A, Guyer M. A taxonomy of $2 \times 2$ games. General Systems 1966;11:203-14.

[72] Traulsen A, Nowak MA, Pacheco JM. Stochastic payoff evaluation increases the temperature of selection. J Theor Biol 2007;244:349-56. 
[73] Nowak MA, May RM. Evolutionary games and spatial chaos. Nature 1992;359:826-9.

[74] Hauert C. Effects of space in $2 \times 2$ games. Int J Bifurc Chaos 2002;12:1531-48.

[75] Santos FC, Pacheco JM, Lenaerts T. Evolutionary dynamics of social dilemmas in structured heterogeneous populations. Proc Natl Acad Sci USA 2006;103:3490-4.

[76] Lozano S, Arenas A, Sánchez A. Mesoscopic structure conditions the emergence of cooperation on social networks. PLoS ONE 2008;3:e1892.

[77] Nowak MA, Bonhoeffer S, May RM. Spatial games and the maintenance of cooperation. Proc Natl Acad Sci USA 1994;91:4877-81.

[78] Lindgren K, Nordahl MG. Evolutionary dynamics of spatial games. Physica D 1994;75:292-309.

[79] Hutson V, Vickers G. The spatial struggle of tit-for-tat and defect. Phil Trans R Soc Lond B 1995;348:393-404.

[80] Grim P. Spatialization and greater generosity in the stochastic prisoner's dilemma. Biosystems 1996;37:3-17.

[81] Nakamaru M, Matsuda H, Iwasa Y. The evolution of cooperation in a lattice-structured population. J Theor Biol 1997;184:65-81.

[82] Szabó G, Tôke C. Evolutionary prisoner's dilemma game on a square lattice. Phys Rev E 1998;58:69-73.

[83] Brauchli K, Killingback T, Doebeli M. Evolution of cooperation in spatially structured populations. J Theor Biol 1999;200:405-17.

[84] Abramson G, Kuperman M. Social games in a social network. Phys Rev E 2001;63:030901(R).

[85] Cohen MD, Riolo RL, Axelrod R. The role of social structure in the maintenance of cooperative regimes. Rationality and Society 2001;13:532.

[86] Vainstein MH, Arenzon JJ. Disordered environments in spatial games. Phys Rev E 2001;64:051905.

[87] Lim Y, Chen K, Jayaprakash C. Scale-invariant behavior in a spatial game of prisoners' dilemma. Phys Rev E 2002;65:026134.

[88] Schweitzer F, Behera L, Mühlenbein H. Evolution of cooperation in a spatial prisoner's dilemma. Adv Complex Sys 2002;5:269-99.

[89] Ifti M, Killingback T, Doebeli M. Effect of neighbourhood size and connectivity on the spatial continuous prisoner's dilemma. J Theor Biol 2004;231:97-106.

[90] Tang C-L, Wang W-X, Wu X, Wang B-H. Effects of average degree on cooperation in networked evolutionary game. Eur Phys J B 2006;53:411-5.

[91] Perc M, Szolnoki A. Social diversity and promotion of cooperation in the spatial prisoner's dilemma. Phys Rev E 2008;77:011904.

[92] Killingback T, Doebeli M. Spatial evolutionary game theory: Hawks and doves revisited. Proc R Soc Lond B 1996;263:1135-44.

[93] Hauert C, Doebeli M. Spatial structure often inhibits the evolution of cooperation in the snowdrift game. Nature 2004;428:643-6.

[94] Sysi-Aho M, Saramäki J, Kertész J, Kaski K. Spatial snowdrift game with myopic agents. Eur Phys J B 2005;44:129-35.

[95] Kun A, Boza G, Scheuring I. Asynchronous snowdrift game with synergistic effect as a model of cooperation. Behav Ecol 2006;17:633-41.

[96] Tomassini M, Luthi L, Giacobini M. Hawks and doves on small-world networks. Phys Rev E 2006;73:016132.

[97] Zhong L-X, Zheng D-F, Zheng B, Xu C, Hui P. Networking effects on cooperation in evolutionary snowdrift game. Europhys Lett 2006;76:724-30.

[98] Blume LE. The statistical mechanics of strategic interaction. Games Econ Behav 1993;5:387-424.

[99] Ellison G. Learning, local interaction, and coordination. Econometrica 1993;61:1047-71.

[100] Kirchkamp O. Spatial evolution of automata in the prisoners' dilemma. J Econ Behav Org 2000;43:239-62.

[101] Roca CP, Cuesta J, Sánchez A. Effect of spatial structure on the evolution of cooperation. Phys Rev E, in press.

[102] Roca CP, Cuesta J, Sánchez A. Promotion of cooperation on networks? The myopic best response case. Eur Phys J B, doi:10.1140/epjb/ e2009-00189-0, in press.

[103] Huberman BA, Glance NS. Evolutionary games and computer simulations. Proc Natl Acad Sci USA 1993;90:7716-8.

[104] Masuda N, Aihara K. Spatial prisoner's dilemma optimally played in small-world networks. Phys Lett A 2003;313:55-61.

[105] Tomochi M. Defectors' niches: Prisoner's dilemma game on disordered networks. Soc Netw 2004;26:309-21.

[106] Hinrichsen H. Non-equilibrium critical phenomena and phase transitions into absorbing states. Adv Phys 2000;49:815-958.

[107] Chiappin JRN, de Oliveira MJ. Emergence of cooperation among interacting individuals. Phys Rev E 1999;59:6419-21.

[108] Newman M. The structure and function of complex networks. SIAM Rev 2003;45:167-256.

[109] Bocaletti S, Latora V, Moreno Y, Chavez M, Hwang DU. Complex networks: Structure and dynamics. Phys Rep 2006;424:175-308.

[110] Watts DJ, Strogatz SH. Collective dynamics of 'small-world' networks. Nature 1998;393:440-2.

[111] Albert R, Barabási A-L. Statistical mechanics of complex networks. Rev Mod Phys 2002;74:47-97.

[112] Holme P, Trusina A, Kim BJ, Minnhagen P. Prisoners dilemma in real-world acquaintance networks: Spikes and quasi-equilibria induced by the interplay between structure and dynamics. Phys Rev E 2003;68:030901.

[113] Guimerà R, Danon L, Díaz-Guilera A, Giralt F, Arenas A. Self-similar community structure in a network of human interactions. Phys Rev E 2003;68:065103.

[114] Helbing D. Interrelations between stochastic equations for systems with pair interactions. Physica A 1992;181:29-52.

[115] Helbing D. A mathematical model for behavioral changes through pair interactions. In: Hagg G, Mueller U, Troitzsch KG, editors. Economic evolution and demographic change. Berlin: Springer-Verlag; 1992. p. 330-48.

[116] Schlag KH. Why imitate, and if so, how? A boundedly rational approach to multi-armed bandits. J Econ Theory 1998;78:130-56.

[117] Blume L. Now noise matters. Games Econ Behav 2003;44:251-71.

[118] Traulsen A, Nowak MA, Pacheco JM. Stochastic dynamics of invasion and fixation. Phys Rev E 2006;74:011909.

[119] Szabó G, Vukov J, Szolnoki A. Phase diagrams for an evolutionary prisoners dilemma game on two-dimensional lattices. Phys Rev E 2005;72:047107.

[120] Luthi L, Tomassini M, Pestelacci E. Evolutionary games on networks and payoff invariance under replicator dynamics. Biosystems 2009;96:213-22.

[121] Roca CP, Cuesta J, Sánchez A. in preparation.

[122] Wu Z-X, Xu X-J, Chen Y, Wang Y-H. Spatial prisoner's dilemma game with volunteering in Newman-Watts small-world networks. Phys Rev E 2005;71:037103. 
[123] Wu Z-X, Xu X-J, Wang Y-H. Prisoner's dilemma game with heterogeneous influential effect on regular small-world networks. Chin Phys Lett 2006;23:531-4.

[124] Cassar A. Coordination and cooperation in local, random and small world networks: Experimental evidence. Games Econ Behav 2007;58:209-30.

[125] Vukov J, Szabó G, Szolnoki A. Cooperation in the noisy case: Prisoner's dilemma game on two types of regular random graphs. Phys Rev E 2006;73:067103.

[126] Hauert C, Szabó G. Game theory and physics. Am J Phys 2005;73:405.

[127] Santos FC, Pacheco JM. Scale-free networks provide a unifying framework for the emergence of cooperation. Phys Rev Lett 2005;95:98104.

[128] Santos FC, Pacheco JM, Lenaerts T. Graph topology plays a determinant role in the evolution of cooperation. Proc Roy Soc B 2006;273:51-6.

[129] Klemm K, Eguíluz VM. Growing scale-free networks with small-world behavior. Phys Rev E 2002;65:057102.

[130] Assenza S, Gómez-Gardeñes J, Latora V. Enhancement of cooperation in highly clustered scale-free networks. Phys Rev E 2009;78:017101.

[131] Gómez-Gardeñes J, Campillo M, Floría LM, Moreno Y. Dynamical organization of cooperation in complex networks. Phys Rev Lett 2007;98:108103.

[132] Poncela J, Gómez-Gardeñes J, Floría LM, Moreno Y. Robustness of cooperation in the prisoner's dilemma in complex networks. New J Phys 2007;9:184.

[133] Nowak MA, Sasaki A, Taylor C, Fudenberg D. Emergence of cooperation and evolutionary stability in finite populations. Nature 2004;428:646-50.

[134] Wild G, Traulsen A. The different limits of weak selection and the evolutionary dynamics of finite populations. J Theor Biol 2007;247:38290.

[135] Ohtsuki H, Nowak MA. The replicator equation on graphs. J Theor Biol 2006;243:86-97.

[136] Challet D, Zhang YC. Emergence of cooperation and organization in an evolutionary game. Physica A 1997;246:407-18.

[137] Zhong L-X, Qiu T, Chen B-H, Liu C-F. Effects of dynamic response time in an evolving market. Physica A 2009;388:673-81.

[138] Zimmermann MG, Eguíluz VM, San Miguel M. Coevolution of dynamical states and interactions in dynamic networks. Phys Rev E 2004;69:65102.

[139] Marsili M, Vega-Redondo F, Slanina F. The rise and fall of a networked society: A formal model. Proc Natl Acad Sci USA 2004;101:1439_ 42.

[140] Eguíluz V, Zimmermann M, Cela-Conde MG, San Miguel M. Cooperation and the emergence of role differentiation in the dynamics of social networks. Am J Sociol 2005;110:977-1008.

[141] Santos FC, Pacheco JM, Lenaerts T. Cooperation prevails when individuals adjust their social ties. PLoS Comput Biol $2006 ; 2:$ e140.

[142] Pacheco JM, Traulsen A, Nowak MA. Coevolution of strategy and structure in complex networks with dynamical linking. Phys Rev Lett 2006;97:258103.

[143] Ohtsuki H, Nowak MA, Pacheco JM. Breaking the symmetry between interaction and replacement in evolutionary dynamics on graphs. Phys Rev Lett 2007;98:108106.

[144] Mengel F, Fosco C. Cooperation through imitation and exclusion in networks. MPRA Paper 5258, University Library of Munich, Germany, Oct. 2007, URL http://ideas.repec.org/p/pra/mprapa/5258.html.

[145] Pacheco JM, Traulsen A, Ohtsuki H, Nowak MA. Repeated games and direct reciprocity under active linking. J Theor Biol 2008;250:723-34.

[146] Gross T, Blasius B. Adaptive coevolutionary networks: A review. J R Soc Interface 2008;5:259-71.

[147] Poncela J, Gómez-Gardeñes J, Floría LM, Sánchez A, Moreno Y. Complex cooperative networks from evolutionary preferential attachment. PLoS ONE 2008;3:e2449.

[148] Sicardi EA, Fort H, Vainstein MH, Arenzon JJ. Random mobility and spatial structure often enhance cooperation. J Theor Biol 2009;256:2406.

[149] Helbing D, Yu W. The outbreak of cooperation among success-driven individuals under noisy conditions. Proc Natl Acad Sci USA 2009;106:3680-5.

[150] Harley CB. Learning the evolutionarily stable strategy. J Theor Biol 1981;89:611-33.

[151] Kirchkamp O. Simultaneous evolution of learning rules and strategies. J Econ Behav Org 1999;40:295-312.

[152] Moyano LG, Sánchez A. Evolving learning rules and emergence of cooperation in spatial prisoner's dilemma. J Theor Biol 2009;259:84-95.

[153] Szabó G, Szolnoki A, Vukov J. Application of Darwinian selection to dynamical rules in spatial prisoners dilemma games. Preprint, URL http://www.mfa.kfki.hu/ szabo/szaborms.html.

[154] Szolnoki A, Perc M. Coevolution of teaching activity promotes cooperation. New J Phys 2008;10:043036.

[155] Szolnoki A, Perc M. Promoting cooperation in social dilemmas via simple coevolutionary rules. Eur Phys J B 2008;67:337-42. 